\title{
A new computational framework for electro-activation in cardiac mechanics
}

\author{
Emilio Garcia-Blanco ${ }^{\mathrm{a}}$, Rogelio Ortigosa ${ }^{\mathrm{b}, *, 1}$, Antonio J. Gil ${ }^{\mathrm{a}, *}$, Chun Hean Lee ${ }^{\mathrm{c}, 2}$, \\ Javier Bonet ${ }^{\mathrm{d}}$ \\ ${ }^{a}$ Zienkiewicz Centre for Computational Engineering, College of Engineering Swansea University, Bay Campus, SA1 8EN, United Kingdom \\ ${ }^{\mathrm{b}}$ Computational Mechanics \& Scientific Computing Group, Technical University of Cartagena, Campus Muralla del Mar, 30202 Cartagena \\ (Murcia), Spain \\ ${ }^{\mathrm{c}}$ Glasgow Computational Engineering Centre, School of Engineering, University of Glasgow, Glasgow, G12 8QQ, United Kingdom \\ ${ }^{\mathrm{d}}$ University of Greenwich, London, SE10 9LS, United Kingdom
}

Received 16 June 2018; received in revised form 10 January 2019; accepted 27 January 2019

Available online 12 February 2019

\begin{abstract}
This paper presents a novel computational framework for the numerical simulation of the electromechanical response of the myocardium during the cardiac cycle. The paper presents the following main novelties. (1) Two new mixed formulations, tailormade for active stress and active strain coupling approaches, have been developed and used in conjunction with two different ionic models, namely Bueno-Orovio et al. (2008) and Ten Tusscher et al. (2004). Taking as a reference the mixed formulations introduced by Bonet et al. (2015) in the context of nonlinear elasticity, the proposed formulations include as unknown fields the geometry and the transmembrane potential (and possibly a Lagrange multiplier enforcing weakly the incompressibility constraint) as well as the deformation gradient tensor, its cofactor, its determinant, the gradient of the transmembrane potential and their respective work conjugates. The Finite Element implementation of these formulations is shown in this paper, where a static condensation procedure is presented in order to yield an extremely competitive computational approach. (2) A comprehensive and rigorous study of different ionic models (i.e Bueno-Orovio and Ten Tusscher) and electromechanical activation couplings (i.e active strain and active stress) has been carried out. (3) An analytical and numerical analysis of the possible loss of ellipticity and polyconvexity of one of the most widely used constitutive models in the context of cardiac mechanics is carried out in this paper, putting forward possible polyconvexifications of the existing model. (4) In addition, an invariant representation of Guccione's constitutive model is proposed. Finally, a series of numerical examples are included in order to demonstrate the applicability and robustness of the proposed formulations.
\end{abstract}

(C) 2019 Elsevier B.V. All rights reserved.

Keywords: Cardiac electromechanics; Mixed formulations; Polyconvexity; Finite elements

\footnotetext{
* Corresponding authors.

E-mail addresses: r.ortigosa@swansea.ac.uk (R. Ortigosa), a.j.gil@swansea.ac.uk (A.J. Gil).

1 This work was completed whilst at the previous affiliation: Zienkiewicz Centre for Computational Engineering, College of Engineering, Swansea University, Bay Campus, SA1 8EN, United Kingdom.

2 This work was completed whilst at the previous affiliation: Zienkiewicz Centre for Computational Engineering, College of Engineering, Swansea University, Bay Campus, SA1 8EN, United Kingdom.
} 


\section{Introduction}

Cardiovascular diseases, such as heart infarction or dysrhythmia, represent the main cause of death in the world [1-3]. Over the last decade, the computational modelling of the complex physical phenomena occurring in the human heart has become an area of increasing scientific interest as this can aid by: (a) providing augmented simulation-based diagnosis tools for patients or innovative surgery techniques for clinicians; (b) gaining better understanding of the mechanisms driving the behaviour of the cardiac muscle both from the physiological and pathological standpoints.

Three aspects are crucial in order to correctly characterise the response of the heart, namely: (a) its passive or purely mechanical behaviour; (b) its electrophysiology; (c) its electric activation, responsible for the electrically driven contraction of the heart. Regarding the first aspect, namely the passive behaviour of the heart, it must be emphasised that the cardiac myocardium is an extremely complex and heterogeneous material with a strongly anisotropic behaviour which can be described via a set of collagen sheets and fibres [4]. To this aim, Diffusion Tensor Magnetic Resonance Imaging (DT-MRI) constitutes a very promising approach [5], although uncertainties related to in vivo measures may lead to unphysiological stress fields [6,7]. Instead, the use of rule-based models or human atlases obtained through statistical treatment of ex vivo DT-MRI scans is preferred [8-10]. In addition, as experimentally reported by Vossoughi et al. [11], the cardiac muscle (or myocardium) is traditionally modelled as a nearly or truly incompressible hyperelastic material although recent investigations [12-14] question these assumptions.

A suitable invariant-based definition of the strain energy encapsulating the passive response of the myocardium must be chosen to describe its passive response, replicating both its anisotropic and incompressible behaviour. Since the pioneering work of Demiray [15], several constitutive models have been proposed [16-21]. In order to ensure a physically admissible behaviour of a constitutive model, this must satisfy the ellipticity condition [22]. A class of functions that satisfy ellipticity is that of polyconvexity [23-31], where the strain energy is defined as a convex multivariable function of the deformation gradient tensor, its cofactor and its determinant. However, to the best of the authors' knowledge, polyconvex constitutive models have not been defined yet for the characterisation of the myocardium.

The second of the three aspects mentioned, namely the electrophysiology of the heart, has also been an intensive area of research [32,33]. The heart fibres contract as a result of electrical stimuli (pulse) initiated in the sinoatrial node, located in the right atrium, and propagated over the heart tissue. This electric pulse produces a sharp rise (depolarisation) followed by a sudden fall (repolarisation) of the transmembrane electric potential in a process known as cardiac action potential [4]. As a result of the depolarisation, a wide range of ion interchanges (electric fluxes) take place across the cell membrane, triggering the cross-bridge cycle [4] in the muscle fibres and leading to the repolarisation of the cell membrane.

The resemblance of the cardiac action potential with propagation of waves enables this phenomenon to be mathematically modelled by means of a convection-diffusion-reaction equation where the source term encapsulates the cellular ion exchange (electric fluxes). For this purpose, a mathematical model named as bidomain model [34-36], was conceived to describe the cardiac action potential by considering two independent electric potentials, namely those in the inner and outer parts of the cell. However, an alternative and simpler approach known as monodomain model [37], where only the transmembrane potential is considered, is usually preferred [7,38-40].

In the Nobel-price winning contributions of Hodgkin and Huxley, the electric fluxes (embedded in the aforementioned source term) were described via a set of ordinary differential equations linking the transmembrane potential with a set of gating variables representing the opening state of the ion channels [32]. Since then, a wide spectrum of more advanced models were developed [41-45]. Priebe and Beuckelmann [46] developed the first human ventricular model able to reproduce physiological and pathological behaviour. In this model, ion concentrations were, in addition to the gating variables in the original model of Hodgkin and Huxley, linked to the transmembrane potential. Moreover, Ten Tusscher et al. [47,48] improved the previous model by considering additional experimental data performed in human ventricular myocytes. Finally, Bueno-Orovio et al. [49] proposed a more simplified model, known as the minimal model, convenient from the computational standpoint. Only the Ten Tusscher and Bueno-Orovio models will be considered in this paper.

The third aspect mentioned, namely the electro-mechanical activation of the heart, occurs as a result of the interaction of calcium ions with Troponin-C during the cross-bridge dynamics [4]. This can be phenomenologically described by means of the so called activation models. Two possible approaches can be followed for the definition of activation models, namely active stress [17] or active strain approaches [50]. Within the first approach, Nash and Panfilov proposed an evolution equation for active cardiomyocite concentration stress [51,52]. Within the 
second approach, Rossi et al. [7] proposed an evolution equation for the electric stretches of the active part of the multiplicatively decomposed deformation gradient tensor.

The computational modelling of the human heart is intrinsically challenging. Even if the three critical aspects described above, namely the passive and active responses of the heart as well as its electrical activation, are perfectly characterised, reliability of the results obtained via numerical simulation can be seriously compromised. Specifically, a computational implementation in terms of displacements and electric potential based on low order Finite Elements, very commonly used among researchers in this area [7,53], are prone to exhibit parasitic behaviour characterised by shear locking and volumetric locking [29,54-57], the latter arising in incompressible scenarios.

In order to circumvent these shortcomings of low order Finite Element implementations, a new mixed formulation is presented in this paper based on a series of recent publications by Gil and Ortigosa in the context of nonlinear elasticity and electro-mechanics [28,58-60]. In this formulation, not only displacements and electric potential (and potentially a pressure-type Lagrange multiplier) are included as unknown fields. In addition, the deformation gradient tensor, its cofactor and its determinant and their respective work conjugates (stress variables) in conjunction with the electric potential gradient and its work conjugate are considered as unknown variables, adding more flexibility into the formulation. In order not to compromise the computational efficiency of the formulation, discontinuous functional spaces across elements are chosen for the extra fields, which enable to condense them out via a static condensation procedure, resulting in an extremely competitive computational approach.

The paper is organised as follows. Section 2 introduces some basic principles of kinematics. Section 3 presents the governing equations describing the motion of the heart and the evolution of the transmembrane potential. In Section 4, relevant aspects concerning the passive response of the myocardium, its electrophysiology and the coupling between the mechanics and the electric physics are presented. Based on the work of Gil and Ortigosa [28,58-60], Section 5 introduces a new mixed formulation in the context of cardiac mechanics and its Finite Element implementation is described in Section 6. A series of examples are included in Section 7 in order to assess the robustness and accuracy of the proposed formulation. Finally, Section 8 provides some concluding remarks and a summary of the key contributions of this paper.

\section{Kinematics}

Let us consider the motion of a continuum (in this context representing the human myocardium) defined by a volume $\Omega_{0}$ with boundary $\partial \Omega_{0}$ in its initial configuration with outward normal $N$. After the motion, the continuum occupies a final configuration defined by a domain $\Omega$ with boundary $\partial \Omega$ with outward normal $\boldsymbol{n}$. The pseudo-time $(t)$ dependent mapping field $\boldsymbol{\phi}$ links a material particle from initial configuration $\boldsymbol{X} \in \Omega_{0}$ to final configuration $\boldsymbol{x} \in \Omega$ according to $\boldsymbol{x}=\boldsymbol{\phi}(\boldsymbol{X}, t)$. The deformation gradient tensor $\boldsymbol{F}_{\boldsymbol{x}}$, its cofactor $\boldsymbol{H}_{\boldsymbol{x}}\left(\boldsymbol{H}_{\boldsymbol{x}}:=\operatorname{Cof} \boldsymbol{F}_{\boldsymbol{x}}\right)$ and its determinant $J_{x}\left(J_{x}:=\operatorname{det} \boldsymbol{F}_{x}\right)$ can be defined as

$$
\boldsymbol{F}_{\boldsymbol{x}}=\nabla_{0} \boldsymbol{x} ; \quad \boldsymbol{H}_{\boldsymbol{x}}=\frac{1}{2} \boldsymbol{F}_{\boldsymbol{x}} \times \boldsymbol{F}_{\boldsymbol{x}} ; \quad J_{\boldsymbol{x}}=\frac{1}{3} \boldsymbol{H}_{\boldsymbol{x}}: \boldsymbol{F}_{\boldsymbol{x}},
$$

where $\nabla_{0}(\bullet)$ denotes the Lagrangian (initial configuration) gradient operator. For any two second order tensors $\boldsymbol{A}$ and $\boldsymbol{B}, \boldsymbol{X}$ in $(1)_{b}$ denotes the tensor cross product operation introduced in de Boer [61] and latter used in the context of nonlinear continuum mechanics [30,31], defined as $(\boldsymbol{A} \times \boldsymbol{B})_{i I}=\mathcal{E}_{i j k} \mathcal{E}_{I J K} A_{j J} B_{k K}$.

As shown in Fig. $1,\left\{\boldsymbol{F}_{\boldsymbol{x}}, \boldsymbol{H}_{\boldsymbol{x}}, J_{\boldsymbol{x}}\right\}$ represent the kinematic measures relating the differential fibre, area and volume elements from initial $\{d \boldsymbol{X}, d \boldsymbol{A}, d V\}$ to final $\{d \boldsymbol{x}, d \boldsymbol{a}, d v\}$ configuration. For virtual and incremental variations of $\boldsymbol{x}$, namely $\delta \boldsymbol{x}$ and $\Delta \boldsymbol{x}$, use of Eq. (1) allows to evaluate the first and second directional derivatives of $\left\{\boldsymbol{F}_{\boldsymbol{x}}, \boldsymbol{H}_{\boldsymbol{x}}, J_{\boldsymbol{x}}\right\}$ as

$$
\begin{aligned}
& D \boldsymbol{F}_{\boldsymbol{x}}[\delta \boldsymbol{x}]=\nabla_{0} \delta \boldsymbol{x} ; \quad D \boldsymbol{H}_{\boldsymbol{x}}[\delta \boldsymbol{x}]=\boldsymbol{F}_{\boldsymbol{x}} \times \nabla_{0} \delta \boldsymbol{x} ; \quad D J_{\boldsymbol{x}}[\delta \boldsymbol{x}]=\boldsymbol{H}_{\boldsymbol{x}}: \boldsymbol{\nabla}_{0} \delta \boldsymbol{x} ; \\
& D^{2} \boldsymbol{F}_{\boldsymbol{x}}[\delta \boldsymbol{x} ; \Delta \boldsymbol{x}]=\mathbf{0} ; \quad D^{2} \boldsymbol{H}_{\boldsymbol{x}}[\delta \boldsymbol{x} ; \Delta \boldsymbol{x}]=\nabla_{0} \delta \boldsymbol{x} \times \nabla_{0} \Delta \boldsymbol{x} ; \quad D^{2} J_{\boldsymbol{x}}[\delta \boldsymbol{x} ; \Delta \boldsymbol{x}]=\boldsymbol{F}_{\boldsymbol{x}}:\left(\nabla_{0} \delta \boldsymbol{x} \times \nabla_{0} \Delta \boldsymbol{x}\right) .
\end{aligned}
$$

\section{Governing equations}

In this section, the coupled system of partial differential equations governing the motion $\boldsymbol{x}$ and the evolution of transmembrane potential $\phi$ in the heart will be described. The first corresponds to the conservation of linear 


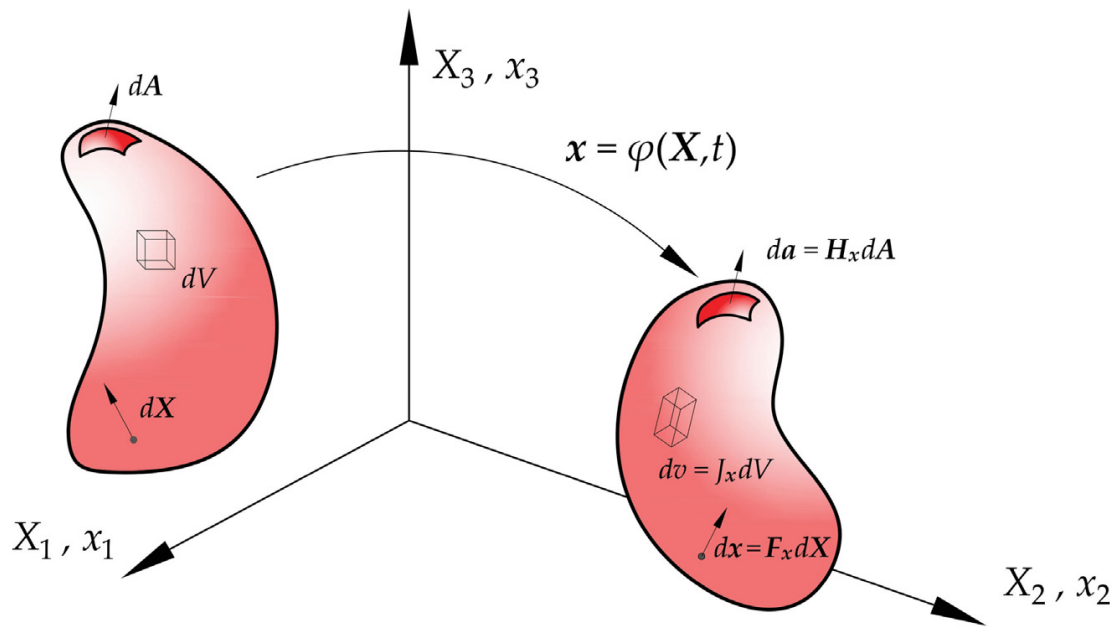

Fig. 1. Motion map of the continuum $\Omega_{0}$ and the kinematic measures $\boldsymbol{F}_{\boldsymbol{x}}, \boldsymbol{H}_{\boldsymbol{x}}, J_{\boldsymbol{x}}$.

momentum (mechanics), ${ }^{3}$ which can be expressed in a Lagrangian setting as

$$
\left.\begin{array}{rlrl}
\operatorname{DIV} \boldsymbol{P}+\boldsymbol{b}_{0} & =\mathbf{0} & & \text { in } \Omega_{0} \times[0, T] \\
\boldsymbol{P} \boldsymbol{N} & =\boldsymbol{t}_{0} & & \text { on } \partial_{t} \Omega_{0} \times[0, T] \\
\boldsymbol{x} & =\boldsymbol{\phi}_{\boldsymbol{x}} & & \text { on } \partial_{\boldsymbol{x}} \Omega_{0} \times[0, T]
\end{array}\right\}
$$

with $\partial \Omega_{0}=\partial_{x} \Omega_{0} \cup \partial_{t} \Omega_{0}$. In Eq. (3), $\boldsymbol{P}$ represents the first Piola-Kirchhoff stress tensor $\boldsymbol{b}_{0}$, a body force per unit undeformed volume and $t_{0}$, a surface force per unit of undeformed area. In addition, the satisfaction of rotational equilibrium leads to the condition $\boldsymbol{P} \boldsymbol{F}_{x}^{T}=\boldsymbol{F}_{\boldsymbol{x}} \boldsymbol{P}^{T}$. The second governing equation represents the time-dependent evolution of the transmembrane potential $\phi$ (electric). When considering a monodomain approach $[37,38,62]$, this can be expressed in a Lagrangian setting as

$$
\left.\begin{array}{rlrl}
\operatorname{DIV} \boldsymbol{Q}+f_{\phi} & =\dot{\phi} & & \text { in } \Omega_{0} \times[0, T] \\
\boldsymbol{Q} \cdot \boldsymbol{N} & =0 & & \text { on } \partial \Omega_{0} \times[0, T] \\
\phi & =\phi_{0}(\boldsymbol{X}) & & \text { in } \Omega_{0}
\end{array}\right\}
$$

where $\phi_{0}(\boldsymbol{X})$ denotes the resting potential (initial conditions) and zero Neumann boundary condition is applied on the boundary $\partial \Omega_{0}$. Moreover, the vector $\boldsymbol{Q}$ represents the electric flux across the cell membrane [53] and $f_{\phi}$, the electrical source term.

\section{Constitutive equations}

For the closure of the governing equations defined by (3) and (4), constitutive laws are needed for the definition of the first Piola-Kirchhoff stress tensor $\boldsymbol{P}$ (3), the electric flux $\boldsymbol{Q}$ (4) and the electrical source term $f_{\phi}(4)$.

\subsection{Passive response of the heart}

The passive response of the heart, encapsulated in the strain energy $\Psi\left(\nabla_{0} x\right)$, can be mathematically described by means of an invariant representation of $\Psi\left(\nabla_{0} \boldsymbol{x}\right)$ in terms of the main kinematic entities $\left\{\boldsymbol{F}_{\boldsymbol{x}}, \boldsymbol{H}_{\boldsymbol{x}}, J_{\boldsymbol{x}}\right\}$, namely

$$
\Psi\left(\nabla_{0} \boldsymbol{x}\right)=W\left(\boldsymbol{F}_{\boldsymbol{x}}, \boldsymbol{H}_{\boldsymbol{x}}, J_{\boldsymbol{x}}\right) .
$$

The energy functional $\Psi\left(\nabla_{0} \boldsymbol{x}\right)$ in (5) has to describe the underlying anisotropic structure of the cardiac tissue, characterised by a spatially varying set of muscle fibres defined by the unitary direction $f_{0}$ in the initial configuration.

\footnotetext{
3 It is customary to neglect inertial effects in Eq. (3) (quasi-statics).
} 

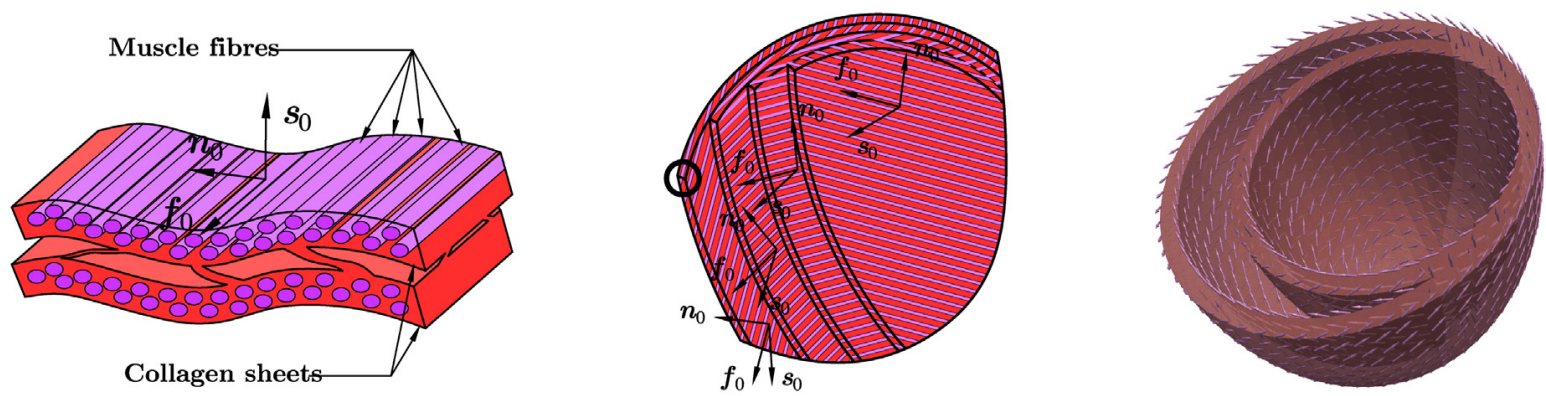

Fig. 2. Anisotropic structure of the cardiac tissue. Representation of the triad $\left\{\boldsymbol{f}_{0}, \boldsymbol{s}_{0}, \boldsymbol{n}_{0}\right\}$ in the Lagrangian setting (left), accounting for their spatial variability (centre). Idealised set of ventricles (right).

These muscle fibres are embedded into the collagen sheets, characterised by the perpendicular unitary normal $s_{0}$ in the initial configuration. This enables to define the triad $\left\{\boldsymbol{f}_{0}, \boldsymbol{s}_{0}, \boldsymbol{n}_{0}\right\}$ at each Lagrangian particle $\boldsymbol{X} \in \Omega_{0}$ (refer to Fig. 2). Furthermore, the functional $\Psi\left(\nabla_{0} \boldsymbol{x}\right)$ needs to account for the incompressible or nearly incompressible nature of the heart tissue $[11,62,63]$.

Anisotropy and incompressibility can be embedded into the extended representation of $\Psi$, namely $W$ (5), by means of an additive decomposition into its isotropic-deviatoric, volumetric and anisotropic contributions, denoted as $\hat{W}, U$ and $W^{\text {ani }}$ respectively, as

$$
W\left(\boldsymbol{F}_{\boldsymbol{x}}, \boldsymbol{H}_{\boldsymbol{x}}, J_{\boldsymbol{x}}, \boldsymbol{f}_{0}, \boldsymbol{s}_{0}\right)=\hat{W}\left(\boldsymbol{F}_{\boldsymbol{x}}, \boldsymbol{H}_{\boldsymbol{x}}, J_{\boldsymbol{x}}\right)+U\left(J_{\boldsymbol{x}}\right)+W^{\mathrm{ani}}\left(\boldsymbol{F}_{\boldsymbol{x}}, \boldsymbol{H}_{\boldsymbol{x}}, J_{\boldsymbol{x}}, \boldsymbol{f}_{0}, \boldsymbol{s}_{0}\right),
$$

where only $\hat{W}$ depends on the isochoric components of $\left\{\boldsymbol{F}_{\boldsymbol{x}}, \boldsymbol{H}_{\boldsymbol{x}}, J_{\boldsymbol{x}}\right\}$ [64-67] namely

$$
\hat{W}\left(\boldsymbol{F}_{\boldsymbol{x}}, \boldsymbol{H}_{\boldsymbol{x}}, J_{\boldsymbol{x}}\right)=W\left(\hat{\boldsymbol{F}}_{\boldsymbol{x}}, \hat{\boldsymbol{H}}_{\boldsymbol{x}}, 1\right) ; \quad \hat{\boldsymbol{F}}_{\boldsymbol{x}}=J_{\boldsymbol{x}}^{-1 / 3} \boldsymbol{F}_{\boldsymbol{x}} ; \quad \hat{\boldsymbol{H}}_{\boldsymbol{x}}=J_{\boldsymbol{x}}^{-2 / 3} \boldsymbol{H}_{\boldsymbol{x}} .
$$

For the purely mechanical physics (no coupling with the transmembrane potential $\phi$ ), the first Piola-Kirchhoff stress tensor $\boldsymbol{P}$ is obtained as

$$
\boldsymbol{P}=\partial_{\nabla_{0} x} \Psi\left(\nabla_{0} \boldsymbol{x}\right) .
$$

Alternatively, the extended representation $\mathrm{W}$ enables to obtain

$$
\boldsymbol{P}: \nabla_{0} \delta \boldsymbol{x}=D W[\delta \boldsymbol{x}]=\left(\partial_{\boldsymbol{F} \boldsymbol{x}} W+\partial_{\boldsymbol{H} \boldsymbol{x}} W \times \boldsymbol{F}_{\boldsymbol{x}}+\partial_{J_{\boldsymbol{x}}} W \boldsymbol{H}_{\boldsymbol{x}}\right): \nabla_{0} \delta \boldsymbol{x},
$$

where use of Eq. (1) has been made in above Eq. (9). Comparison of Eqs. (8) and (9) enables to obtain an alternative expression for the first Piola-Kirchhoff stress tensor as

$$
\boldsymbol{P}=\partial_{\boldsymbol{F}_{\boldsymbol{x}}} W+\partial_{\boldsymbol{H}_{\boldsymbol{x}}} W \times \boldsymbol{F}_{\boldsymbol{x}}+\partial_{J_{\boldsymbol{x}}} W \boldsymbol{H}_{\boldsymbol{x}},
$$

The ellipticity (rank-one convexity) or material stability of the strain energy $\Psi\left(\nabla_{0} x\right)$ guarantees the wellposedness of Eq. (3). This condition is directly related to the tangent operator of the strain energy $\Psi\left(\nabla_{0} x\right)$, i.e.

$$
D^{2} \Psi[\delta \boldsymbol{x} ; \Delta \boldsymbol{x}]=D\left(\boldsymbol{P}: \nabla_{0} \delta \boldsymbol{x}\right)[\Delta \boldsymbol{x}]=\nabla_{0} \delta \boldsymbol{x}: \mathcal{C}: \nabla_{0} \Delta \boldsymbol{x} ; \quad \mathcal{C}=\partial_{\nabla_{0} x \nabla_{0} x}^{2} \Psi,
$$

where $\mathcal{C}$ represents the fourth order elasticity tensor. Alternatively, the tangent operator can be equivalently obtained in terms of the extended representation $W\left(\boldsymbol{F}_{\boldsymbol{x}}, \boldsymbol{H}_{\boldsymbol{x}}, J_{\boldsymbol{x}}\right)$ as

$$
\begin{aligned}
& D^{2} W[\delta \boldsymbol{x} ; \Delta \boldsymbol{x}]=D\left(\boldsymbol{P}: \nabla_{0} \delta \boldsymbol{x}\right)[\Delta \boldsymbol{x}]=\left[\begin{array}{c}
\nabla_{0} \delta \boldsymbol{x}: \\
\boldsymbol{F}_{\boldsymbol{x}} \times \nabla_{0} \delta \boldsymbol{x}: \\
\boldsymbol{H}_{\boldsymbol{x}}: \nabla_{0} \delta \boldsymbol{x}
\end{array}\right]^{T}\left[\mathbb{H}_{W}\right]\left[\begin{array}{c}
: \nabla_{0} \Delta \boldsymbol{x} \\
: \boldsymbol{F}_{\boldsymbol{x}} \times \nabla_{0} \Delta \boldsymbol{x} \\
\boldsymbol{H}_{\boldsymbol{x}}: \nabla_{0} \Delta \boldsymbol{x}
\end{array}\right] \\
& +\left(\partial_{\boldsymbol{H}_{\boldsymbol{x}}} W+\partial_{J_{\boldsymbol{x}}} W \boldsymbol{F}_{\boldsymbol{x}}\right):\left(\nabla_{0} \delta \boldsymbol{x} \times \nabla_{0} \Delta \boldsymbol{x}\right) \text {, }
\end{aligned}
$$


where $\left[\mathbb{H}_{W}\right]$ represents the Hessian operator of $\mathrm{W}$, namely

$$
\left[\mathbb{H}_{W}\right]=\left[\begin{array}{ccc}
\partial_{\boldsymbol{F}_{\boldsymbol{x}} \boldsymbol{F}_{\boldsymbol{x}}}^{2} W & \partial_{\boldsymbol{F}_{\boldsymbol{x}} \boldsymbol{H}_{\boldsymbol{x}}}^{2} W & \partial_{\boldsymbol{F}_{\boldsymbol{x}} J_{\boldsymbol{x}}}^{2} W \\
\partial_{\boldsymbol{H}_{\boldsymbol{x}} \boldsymbol{F}_{\boldsymbol{x}}}^{2} W & \partial_{\boldsymbol{H}_{\boldsymbol{x}} \boldsymbol{H}_{\boldsymbol{x}}}^{2} W & \partial_{\boldsymbol{H}_{\boldsymbol{x}} J_{\boldsymbol{x}}}^{2} W \\
\partial_{\boldsymbol{J}_{\boldsymbol{x}} \boldsymbol{F}_{\boldsymbol{x}}}^{2} W & \partial_{\boldsymbol{J}_{\boldsymbol{x}} \boldsymbol{H}_{\boldsymbol{x}}}^{2} W & \partial_{\boldsymbol{J}_{\boldsymbol{x}} \boldsymbol{J}_{\boldsymbol{x}}}^{2} W
\end{array}\right] .
$$

Comparison of (11) and (12) enables to additively decompose $\mathcal{C}$ into a purely material contribution $\mathcal{C}_{m}$ (depending upon second derivatives of $W$ ) and a geometrical contribution $\mathcal{C}_{g}$ (emerging from the inherent non-linearity of $\left.\left\{\boldsymbol{H}_{\boldsymbol{x}}, J_{\boldsymbol{x}}\right\}\right)$, namely $\mathcal{C}=\mathcal{C}_{m}+\mathcal{C}_{g}$, with

$$
\begin{aligned}
\mathcal{C}_{m} & =\partial_{\boldsymbol{F}_{\boldsymbol{x}} \boldsymbol{F}_{\boldsymbol{x}}}^{2} W+\boldsymbol{F}_{\boldsymbol{x}} \times \partial_{\boldsymbol{H}_{\boldsymbol{x}} \boldsymbol{H}_{\boldsymbol{x}}}^{2} W \times \boldsymbol{F}_{\boldsymbol{x}}+\partial_{J_{\boldsymbol{x}} J_{\boldsymbol{x}}}^{2} W \boldsymbol{H}_{\boldsymbol{x}} \otimes \boldsymbol{H}_{\boldsymbol{x}}+\partial_{\boldsymbol{F}_{\boldsymbol{x}} \boldsymbol{H}_{\boldsymbol{x}}}^{2} W \times \boldsymbol{F}_{\boldsymbol{x}}+\boldsymbol{F}_{\boldsymbol{x}} \times \partial_{\boldsymbol{H}_{\boldsymbol{x}} \boldsymbol{F}_{\boldsymbol{x}}}^{2} W \\
& +\partial_{\boldsymbol{F}_{\boldsymbol{x}} J_{\boldsymbol{x}}}^{2} W \otimes \boldsymbol{H}_{\boldsymbol{x}}+\boldsymbol{H}_{\boldsymbol{x}} \otimes \partial_{J_{\boldsymbol{x}} \boldsymbol{F}_{\boldsymbol{x}}}^{2} W+\boldsymbol{F}_{\boldsymbol{x}} \times \partial_{\boldsymbol{H}_{\boldsymbol{x}} J_{\boldsymbol{x}}}^{2} W \otimes \boldsymbol{H}_{\boldsymbol{x}}+\boldsymbol{H}_{\boldsymbol{x}} \otimes \partial_{J_{\boldsymbol{x}} \boldsymbol{H}_{\boldsymbol{x}}}^{2} W \times \boldsymbol{F}_{\boldsymbol{x}} \\
\mathcal{C}_{g} & =\mathcal{I} \times\left(\partial_{\boldsymbol{H}_{\boldsymbol{x}}} W+\partial_{J_{\boldsymbol{x}}} W \boldsymbol{F}_{\boldsymbol{x}}\right),
\end{aligned}
$$

with $\mathcal{I}_{i I j J}=\delta_{i j} \delta_{I J}$ and $(\mathcal{A} \times \boldsymbol{A})_{i I j J}=\mathcal{E}_{j p q} \mathcal{E}_{J P Q} \mathcal{A}_{i I p P} A_{q Q}$ and $(\boldsymbol{A} \times \mathcal{A})_{i I j J}=\mathcal{E}_{i p q} \mathcal{E}_{I P Q} A_{p P} \mathcal{A}_{q Q j J}$, for $\mathcal{A} \in$ $\mathbb{R}^{3 \times 3 \times 3 \times 3}$ and $\boldsymbol{A} \in \mathbb{R}^{3 \times 3}$.

The ellipticity condition requires that, for $\nabla_{0} \delta \boldsymbol{x}=\nabla_{0} \Delta \boldsymbol{x}=\boldsymbol{u} \otimes \boldsymbol{V}$, with $\boldsymbol{u}, \boldsymbol{V} \in \mathbb{R}^{3}$ and $\boldsymbol{u} \neq \mathbf{0}, \boldsymbol{V} \neq \mathbf{0}$, the tangent operator in (11) and (12) is positive, namely

$$
\mathcal{I}_{W}=(\boldsymbol{u} \otimes \boldsymbol{V}): \mathcal{C}:(\boldsymbol{u} \otimes \boldsymbol{V})=\left[\begin{array}{c}
\boldsymbol{u} \otimes \boldsymbol{V}: \\
\boldsymbol{F}_{\boldsymbol{x}} \mathbf{x}(\boldsymbol{u} \otimes \boldsymbol{V}): \\
\boldsymbol{H}_{\boldsymbol{x}}:(\boldsymbol{u} \otimes \boldsymbol{V})
\end{array}\right]^{T}\left[\mathbb{H}_{W}\right]\left[\begin{array}{c}
: \boldsymbol{u} \otimes \boldsymbol{V} \\
: \boldsymbol{F}_{\boldsymbol{x}} \times(\boldsymbol{u} \otimes \boldsymbol{V}) \\
\boldsymbol{H}_{\boldsymbol{x}}:(\boldsymbol{u} \otimes \boldsymbol{V})
\end{array}\right] \geq 0
$$

In above Eq. (15), the contribution from the geometrical component $\mathcal{C}_{g}$ vanishes, i.e. $(\boldsymbol{u} \otimes \boldsymbol{V}): \mathcal{C}_{g}:(\boldsymbol{u} \otimes \boldsymbol{V})=0$, as shown in References [58]. A sufficient condition for (15) to hold is the positive definiteness of the acoustic tensor $\mathcal{C}_{V V}$, defined as

$$
\left(\mathcal{C}_{V V}\right)_{i j}=(\mathcal{C})_{i I j J} V_{I} V_{J} ; \quad \forall \boldsymbol{V} \in \mathbb{R}^{3} ; \quad \boldsymbol{V} \neq \mathbf{0} .
$$

Alternatively, a more restrictive sufficient condition for (15) to hold is the positive definiteness of $\left[\mathbb{H}_{W}\right]$. This is automatically satisfied if the strain energy functional $\Psi\left(\nabla_{0} \boldsymbol{x}\right)(5)$ is sufficiently differentiable and polyconvex [23,24,26,27,68], namely if its extended representation $W\left(\boldsymbol{F}_{\boldsymbol{x}}, \boldsymbol{H}_{\boldsymbol{x}}, \boldsymbol{J}_{\boldsymbol{x}}\right)$ is convex with respect to its arguments, i.e.

$$
W\left(\boldsymbol{A}_{3}, \boldsymbol{B}_{3}, C_{3}\right) \leq \lambda W\left(\boldsymbol{A}_{1}, \boldsymbol{B}_{1}, C_{1}\right)+(1-\lambda) W\left(\boldsymbol{A}_{2}, \boldsymbol{B}_{2}, C_{2}\right) ; \quad \forall \lambda \in[0,1],
$$

$\forall \boldsymbol{A}_{1}, \boldsymbol{A}_{2}, \boldsymbol{B}_{1}, \boldsymbol{B}_{2} \in \mathbb{R}^{3 \times 3}$ and $C_{1}, C_{2} \in \mathbb{R}$ and $\boldsymbol{A}_{3}=\lambda \boldsymbol{A}_{1}+(1-\lambda) \boldsymbol{A}_{2}, \boldsymbol{B}_{3}=\lambda \boldsymbol{B}_{1}+(1-\lambda) \boldsymbol{B}_{2}$ and $C_{3}=$ $\lambda C_{1}+(1-\lambda) C_{2}$.

\subsubsection{An example for the passive response}

As an example, let us consider the classical invariant-based representation of the strain energy proposed by Holzapfel-Ogden [21] for the characterisation of the passive response of the myocardium. In this energy, each one of the terms introduced in Eq. (6) are

$$
\begin{array}{cc}
\hat{W}\left(\boldsymbol{F}_{\boldsymbol{x}}, J_{\boldsymbol{x}}\right)=\frac{a}{2 b} e^{b\left(I I_{\hat{\boldsymbol{F}}_{x}}-3\right)} ; & U\left(J_{\boldsymbol{x}}\right)=\frac{\kappa}{2}\left(J_{\boldsymbol{x}}-1\right)^{2} ; \\
W^{\text {ani }}\left(\boldsymbol{F}_{\boldsymbol{x}}, \boldsymbol{f}_{0}, \boldsymbol{s}_{0}\right)=\frac{a_{f}}{2 b_{f}}\left(e^{b_{f}\left\langle I_{4, f_{0}}-1\right\rangle^{2}}-1\right)+\frac{a_{s}}{2 b_{s}}\left(e^{b_{s}\left\langle I_{4, s_{0}}-1\right\rangle^{2}}-1\right)+\frac{a_{f s}}{2 b_{f s}}\left(e^{b_{f s} I_{8, f_{0} s_{0}}^{2}}-1\right),
\end{array}
$$

where $\left\{a, \kappa, a_{f}, a_{s}, a_{f s}, b_{f}, b_{s}, b_{f s}\right\}$ are positive material constants and $\langle\bullet\rangle$ represents the Macaulay brackets $\langle\bullet\rangle=$ $(\bullet+|\bullet|) / 2$ to prevent fibres from working in contraction [69,70], $I I_{(\bullet)}$ denotes the second invariant of $(\bullet)$ and with the anisotropic invariants $I_{4, f_{0}}, I_{4, s_{0}}, I_{8, f_{0} s_{0}}$ defined as

$$
I_{4, f_{0}}=F_{x} f_{0} \cdot F_{x} f_{0} ; \quad I_{4, s_{0}}=F_{x} s_{0} \cdot F_{x} s_{0} ; \quad I_{8, f_{0} s_{0}}=F_{x} f_{0} \cdot F_{x} s_{0} .
$$


For the specific constitutive model defined in (18), the elements of the set $\left\{\partial_{\boldsymbol{F}_{\boldsymbol{x}}} W, \partial_{\boldsymbol{H}_{\boldsymbol{x}}} W, \partial_{J_{\boldsymbol{x}}} W\right\}$ featuring in the definition of $\boldsymbol{P}$ in (10) are

$$
\begin{aligned}
& \partial_{\boldsymbol{F}_{\boldsymbol{x}}} W=a e^{b\left(I \hat{\boldsymbol{F}}_{x}-3\right)} J_{\boldsymbol{x}}^{-\frac{2}{3}} \boldsymbol{F}_{\boldsymbol{x}}+2 a_{f} e^{b_{f}\left(I_{4, f_{0}}-1\right)^{2}}\left\langle I_{4, f_{0}}-1\right\rangle \boldsymbol{F}_{\boldsymbol{x}} \boldsymbol{f}_{0} \otimes \boldsymbol{f}_{0} \\
& +2 a_{s} e^{b_{s}\left\langle I_{4, s_{0}}-1\right)^{2}}\left\langle I_{4, s_{0}}-1\right\rangle \boldsymbol{F}_{\boldsymbol{x}} \boldsymbol{s}_{0} \otimes \boldsymbol{s}_{0}+a_{f s} e^{b_{f s} I_{8, f_{0} s_{0}}^{2}} I_{8, f_{0} s_{0}}\left(\boldsymbol{F}_{\boldsymbol{x}} \boldsymbol{f}_{0} \otimes \boldsymbol{s}_{0}+\boldsymbol{F}_{\boldsymbol{x}} \boldsymbol{s}_{0} \otimes \boldsymbol{f}_{0}\right) ; \\
& \partial_{\boldsymbol{H}_{\boldsymbol{x}}} W=\mathbf{0} \\
& \partial_{J_{\boldsymbol{x}}} W=-\frac{a}{3} J_{\boldsymbol{x}}^{-1} I I_{\hat{\boldsymbol{F}}_{x}} e^{b\left(I I_{\hat{\boldsymbol{F}}_{x}}-3\right)}+U^{\prime}\left(J_{\boldsymbol{x}}\right) .
\end{aligned}
$$

The energy functional in (18) is not elliptic and, hence, not polyconvex. This is shown in Appendix A, where the following expression for the ellipticity indicator $\mathcal{I}_{W}(15)$ has been obtained

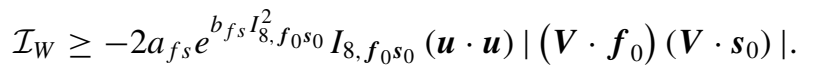

From (21), it can be concluded that the only term responsible for the possible loss of ellipticity of the model in (18) is that associated with the non-elliptic invariant $I_{8, f_{0} s_{0}} . \mathcal{I}_{W}$ (15) might adopt negative values throughout the deformation due to this term. Negative values of $\mathcal{I}_{W}$ (21) for any given $\boldsymbol{u}, \boldsymbol{V} \in \mathbb{R}^{3}, \boldsymbol{u}, \boldsymbol{V} \neq \mathbf{0}$ can induce loss of positive definiteness of the acoustic tensor $\mathcal{C}_{V V}$ in (16), leading to loss of ellipticity and, hence, loss of polyconvexity. A numerical study of the possible loss of ellipticity and polyconvexity of this model will be carried out in Section 7.6.

Remark 1. Notice that it is possible to (poly)-convexify the invariant $I_{8, f_{0} s_{0}}$ via its convexified counterpart $I_{8, f_{0} s_{0}}^{\star}$ defined as

$$
I_{8, f_{0} s_{0}}^{\star}=\left(\alpha \boldsymbol{F}_{\boldsymbol{x}} \boldsymbol{f}_{0}+\beta \boldsymbol{F}_{\boldsymbol{x}} \boldsymbol{s}_{0}\right) \cdot\left(\alpha \boldsymbol{F}_{\boldsymbol{x}} \boldsymbol{f}_{0}+\beta \boldsymbol{F}_{\boldsymbol{x}} \boldsymbol{s}_{0}\right)-\left(\alpha^{2}+\beta^{2}\right),
$$

where the dimensionless parameters $\{\alpha, \beta\}$ would have to be defined via material characterisation. Replacing $I_{8, f_{0} s_{0}}$ with $I_{8, f_{0} s_{0}}^{\star}$ in (18) yields a polyconvexification of this constitutive model. The specific choice of $\alpha=1$ and $\beta=-1$ is of special interest as the resulting invariant represents the stretch or shortening of the hypotenuse of the right-angled triangle with adjacent sides defined by the unit vectors $f_{0}$ and $s_{0}$.

In the following sections, two well-known approaches describing the coupling of the strain energy $W$ in Eq. (18) with the electro-activation will be presented.

\subsubsection{Coupling of first Piola-Kirchhoff stress tensor with the transmembrane potential $\phi:$ active stress approach}

In the active stress approach [17,51,52], an additive decomposition of $\boldsymbol{P}$ is defined in terms of a coupled (active) $\boldsymbol{P}_{\text {Active }}$ contribution and a purely mechanical (passive) $\boldsymbol{P}_{\text {Passive }}$ contribution as

$$
\boldsymbol{P}=\boldsymbol{P}_{\text {Active }}+\boldsymbol{P}_{\text {Passive }} ; \quad \boldsymbol{P}_{\text {Active }}=T_{a} \boldsymbol{F}_{\boldsymbol{x}} \boldsymbol{f}_{0} \otimes \boldsymbol{f}_{0} ; \quad \boldsymbol{P}_{\text {Passive }}=\partial_{\boldsymbol{F}_{\boldsymbol{x}}} W+\partial_{\boldsymbol{H}_{\boldsymbol{x}}} W \times \boldsymbol{F}_{\boldsymbol{x}}+\partial_{J_{\boldsymbol{x}}} W \boldsymbol{H}_{\boldsymbol{x}},
$$

where $T_{a}$ represents the active cardiomyocite contraction stress and the passive term $\boldsymbol{P}_{\text {Passive }}$ adopts an identical representation to that in (10). Evolution equations relating $T_{a}$ with the transmembrane potential $\phi$ will be presented in Section 4.3.1.

In this approach, the tangent operator of the active contribution $\boldsymbol{P}_{\text {Active }}$ can be written as

$$
D\left(\boldsymbol{P}_{\text {Active }}: \nabla_{0} \delta \boldsymbol{x}\right)[\Delta \boldsymbol{x}]=\nabla_{0} \delta \boldsymbol{x}:\left[T_{a} \mathcal{T}_{f_{0} f_{0}}\right]: \nabla_{0} \Delta \boldsymbol{x} ; \quad\left(\mathcal{T}_{f_{0} f_{0}}\right)_{i I j J}=\delta_{i j}\left(f_{0}\right)_{I}\left(f_{0}\right)_{J} .
$$

Therefore, in addition to the material and geometrical components $\mathcal{C}_{m}$ and $\mathcal{C}_{g}$, the elasticity tensor includes an additional active contribution $\mathcal{C}_{T_{a}}$ as

$$
\mathcal{C}=\mathcal{C}_{m}+\mathcal{C}_{g}+\mathcal{C}_{T_{a}} ; \quad \mathcal{C}_{T_{a}}=T_{a} \mathcal{T}_{f_{0} f_{0}}
$$

The ellipticity condition for the active stress approach ${ }^{4}$ can then be written as

$$
(\boldsymbol{u} \otimes \boldsymbol{V}): \mathcal{C}:(\boldsymbol{u} \otimes \boldsymbol{V})=\left[\begin{array}{c}
\boldsymbol{u} \otimes \boldsymbol{V}: \\
\boldsymbol{F}_{\boldsymbol{x}} \times(\boldsymbol{u} \otimes \boldsymbol{V}): \\
\boldsymbol{H}_{\boldsymbol{x}}:(\boldsymbol{u} \otimes \boldsymbol{V})
\end{array}\right]^{T}\left[\widetilde{\mathbb{H}}_{W}\right]\left[\begin{array}{c}
: \boldsymbol{u} \otimes \boldsymbol{V} \\
: \boldsymbol{F}_{\boldsymbol{x}} \times(\boldsymbol{u} \otimes \boldsymbol{V}) \\
\boldsymbol{H}_{\boldsymbol{x}}:(\boldsymbol{u} \otimes \boldsymbol{V})
\end{array}\right] \geq 0,
$$

\footnotetext{
${ }^{4}$ Taking $\nabla_{0} \delta \boldsymbol{x}=\nabla_{0} \Delta \boldsymbol{x}=\boldsymbol{u} \otimes \boldsymbol{V}$.
} 


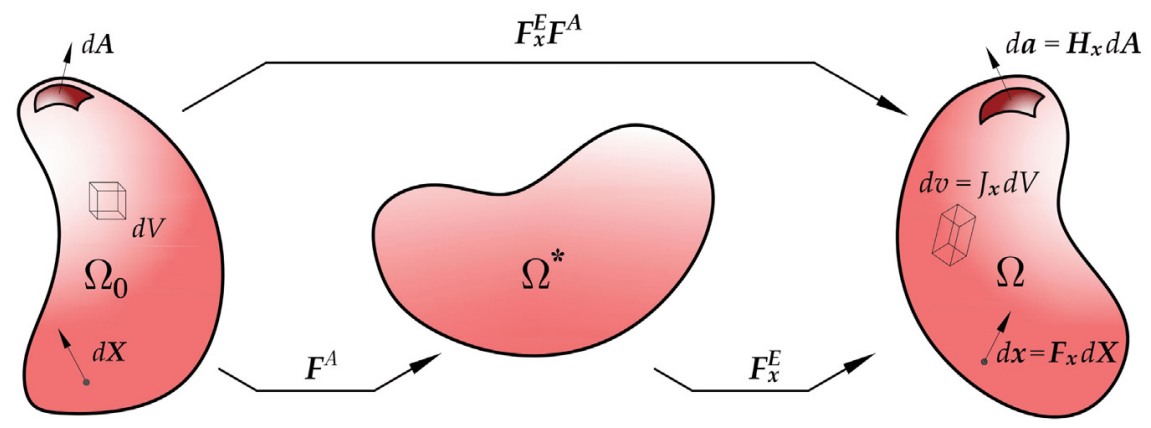

Fig. 3. Multiplicative decomposition of the deformation gradient tensor $\boldsymbol{F}_{\boldsymbol{x}}$ for the active strain approach.

with

$$
\left[\widetilde{\mathbb{H}}_{W}\right]=\left[\mathbb{H}_{W}\right]+\left[\begin{array}{ccc}
T_{a} \boldsymbol{f}_{0} \otimes \boldsymbol{f}_{0} & \mathbf{0}_{3 \times 3} & \mathbf{0}_{3 \times 1} \\
\mathbf{0}_{3 \times 3} & \mathbf{0}_{3 \times 3} & \mathbf{0}_{3 \times 1} \\
\mathbf{0}_{1 \times 3} & \mathbf{0}_{1 \times 3} & 0
\end{array}\right] .
$$

Crucially, positive values of $T_{a}$ (associated with contractions along the fibre $f_{0}$ ) guarantee the ellipticity of the active contribution $\boldsymbol{P}_{\text {Active }}$ in $(23)_{b}$. Furthermore, positive values of $T_{a}$ can help stabilising the loss of ellipticity induced by the energetic contribution associated with the invariant $I_{8, f_{0} s_{0}}$ in (18) (refer to end of Section 4.1.1).

\subsubsection{Coupling of stresses with the transmembrane potential $\phi$ : active strain approach}

The second approach for the coupling of the mechanical and electrical physics, known as active strain [7,50,62], is based on a multiplicative decomposition of the deformation gradient tensor $\boldsymbol{F}_{\boldsymbol{x}}$ into its elastic (passive) $\boldsymbol{F}_{\boldsymbol{x}}^{E}$ and coupled (active) $\boldsymbol{F}^{A}$ contributions (refer to Fig. 3), namely

$$
\boldsymbol{F}_{\boldsymbol{x}}=\boldsymbol{F}_{\boldsymbol{x}}^{E} \boldsymbol{F}^{A} .
$$

In this approach, the coupling is embedded into the active component $\boldsymbol{F}^{A}$, related to the electrically activated stretches $\left\{\gamma_{f_{0}}, \gamma_{s_{0}}, \gamma_{n_{0}}\right\}$ along the fibres $\left\{\boldsymbol{f}_{0}, \boldsymbol{s}_{0}, \boldsymbol{n}_{0}\right\}$, defined as

$$
\boldsymbol{F}^{A}=\boldsymbol{I}+\gamma_{f_{0}} \boldsymbol{f}_{0} \otimes \boldsymbol{f}_{0}+\gamma_{s_{0}} \boldsymbol{s}_{0} \otimes \boldsymbol{s}_{0}+\gamma_{\boldsymbol{n}_{0}} \boldsymbol{n}_{0} \otimes \boldsymbol{n}_{0}
$$

Evolution equations relating $\gamma_{f_{0}}$ with the transmembrane potential $\phi$ will be presented in Section 4.3.2. The $\left\{\gamma_{s_{0}}, \gamma_{n_{0}}\right\}$ are usually related to $\gamma_{f_{0}}$ (see Remark 2).

Remark 2. It is customary [62,71] to assume that $\operatorname{det} \boldsymbol{F}^{A}=1$. Therefore, the widely used assumption of equal values for $\left\{\gamma_{f_{0}}, \gamma_{s_{0}}\right\}$ enables to relate both to $\gamma_{f_{0}}$ as

$$
\gamma_{s_{0}}=\gamma_{n_{0}}=\frac{1}{\sqrt{1+\gamma_{f_{0}}}}-1
$$

Nevertheless, the latter assumption fails to correctly reproduce the heart wall thickness reduction during a heartbeat [13]. In order to circumvent this shortcoming, Rossi et al. [7] proposed an alternative definition of $\left\{\gamma_{f_{0}}, \gamma_{s_{0}}\right\}$ (compatible with $\operatorname{det} \boldsymbol{F}^{A}=1$ ) as

$$
\gamma_{n_{0}}=4 \gamma_{f_{0}} ; \quad \gamma_{s_{0}}=\frac{1}{\left(1+\gamma_{f_{0}}\right)\left(1+\gamma_{n_{0}}\right)}-1 .
$$

In the active strain approach, the coupling is achieved after a redefinition of the strain energy $\Psi$, which must depend on the elastic components of $\boldsymbol{F}_{\boldsymbol{x}}$, namely

$$
\Psi\left(\nabla_{0} \boldsymbol{x}, \boldsymbol{F}^{A}\right)=W\left(\boldsymbol{F}_{\boldsymbol{x}}^{E}, \boldsymbol{H}_{\boldsymbol{x}}^{E}, J_{\boldsymbol{x}}^{E}\right),
$$

with $\left\{\boldsymbol{F}_{\boldsymbol{x}}^{E}, \boldsymbol{H}_{\boldsymbol{x}}^{E}, J_{\boldsymbol{x}}^{E}\right\}$ defined as

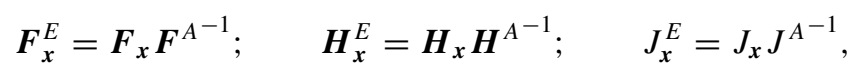


and

$$
\boldsymbol{H}^{A}=\frac{1}{2} \boldsymbol{F}^{A} \times \boldsymbol{F}^{A} ; \quad J^{A}=\frac{1}{3} \boldsymbol{H}^{A}: \boldsymbol{F}^{A} .
$$

This enables to re-express the strain energy in (32) in terms of the strain measures $\left\{\boldsymbol{F}_{\boldsymbol{x}}, \boldsymbol{H}_{\boldsymbol{x}}, J_{\boldsymbol{x}}\right\}$ and its active counterparts $\left\{\boldsymbol{F}^{A}, \boldsymbol{H}^{A}, J^{A}\right\}$ as

$$
\Psi\left(\nabla_{0} \boldsymbol{x}\right)=W\left(\boldsymbol{F}_{\boldsymbol{x}}^{E}, \boldsymbol{H}_{\boldsymbol{x}}^{E}, J_{\boldsymbol{x}}^{E}\right)=W^{E}\left(\boldsymbol{F}_{\boldsymbol{x}}, \boldsymbol{H}_{\boldsymbol{x}}, J_{\boldsymbol{x}}, \boldsymbol{F}^{A^{-1}}, \boldsymbol{H}^{A^{-1}}, J^{A^{-1}}\right) .
$$

The re-expression of the strain energy in terms of $\left\{\boldsymbol{F}_{\boldsymbol{x}}, \boldsymbol{H}_{\boldsymbol{x}}, J_{\boldsymbol{x}}\right\}$ leads to an expression for $\boldsymbol{P}$ as

$$
\boldsymbol{P}=\partial_{\boldsymbol{F}_{\boldsymbol{x}}} W^{E}+\partial_{\boldsymbol{H}_{\boldsymbol{x}}} W^{E} \times \boldsymbol{F}_{\boldsymbol{x}}+\partial_{J_{\boldsymbol{x}}} W^{E} \boldsymbol{H}_{\boldsymbol{x}} .
$$

The tangent operator of $W^{E}$ is

$$
\begin{aligned}
& D^{2} W[\delta \boldsymbol{x} ; \Delta \boldsymbol{x}]=D^{2} W^{E}[\delta \boldsymbol{x} ; \Delta \boldsymbol{x}]=\left[\begin{array}{c}
\boldsymbol{\nabla}_{0} \delta \boldsymbol{x}: \\
\boldsymbol{F}_{\boldsymbol{x}} \times \nabla_{0} \delta \boldsymbol{x}: \\
\boldsymbol{H}_{\boldsymbol{x}}: \boldsymbol{\nabla}_{0} \delta \boldsymbol{x}
\end{array}\right]^{T}\left[\mathbb{H}_{W^{E}}\right]\left[\begin{array}{c}
: \nabla_{0} \Delta \boldsymbol{x} \\
: \boldsymbol{F}_{\boldsymbol{x}} \times \nabla_{0} \Delta \boldsymbol{x} \\
\boldsymbol{H}_{\boldsymbol{x}}: \nabla_{0} \Delta \boldsymbol{x}
\end{array}\right] \\
& +\left(\partial_{\boldsymbol{H}_{\boldsymbol{x}}} W^{E}+\partial_{J_{\boldsymbol{x}}} W^{E} \boldsymbol{F}_{\boldsymbol{x}}\right):\left(\nabla_{0} \delta \boldsymbol{x} \times \nabla_{0} \Delta \boldsymbol{x}\right) \text {, }
\end{aligned}
$$

where $\left[\mathbb{H}_{W^{E}}\right]$ represents the Hessian operator of $W^{E}$. The ellipticity indicator $\mathcal{I}_{W}$ can now be expressed as

$$
\mathcal{I}_{W}=(\boldsymbol{u} \otimes \boldsymbol{V}): \mathcal{C}_{W^{E}}:(\boldsymbol{u} \otimes \boldsymbol{V})=\left[\begin{array}{c}
\boldsymbol{u} \otimes \boldsymbol{V}: \\
\boldsymbol{F}_{\boldsymbol{x}} \times(\boldsymbol{u} \otimes \boldsymbol{V}): \\
\boldsymbol{H}_{\boldsymbol{x}}:(\boldsymbol{u} \otimes \boldsymbol{V})
\end{array}\right]^{T}\left[\mathbb{H}_{W E}\right]\left[\begin{array}{c}
: \boldsymbol{u} \otimes \boldsymbol{V} \\
: \boldsymbol{F}_{\boldsymbol{x}} \times(\boldsymbol{u} \otimes \boldsymbol{V}) \\
\boldsymbol{H}_{\boldsymbol{x}}:(\boldsymbol{u} \otimes \boldsymbol{V})
\end{array}\right] \geq 0 .
$$

In this approach, the consideration of the strain energy $W^{E}$ as in (35) leads to re-expression of the model in Eq. (18) as

$$
\begin{array}{cc}
\hat{W}^{E}\left(\boldsymbol{F}_{\boldsymbol{x}}, J_{\boldsymbol{x}}, \boldsymbol{F}^{A^{-1}}\right)=\frac{a}{2 b} e^{b\left(I I_{\hat{\boldsymbol{F}}_{x}}^{E}-3\right)} ; & U^{E}\left(J_{\boldsymbol{x}} J^{A^{-1}}\right)=\frac{\kappa}{2}\left(J_{\boldsymbol{x}} J^{A^{-1}}-1\right)^{2} ; \\
W^{E \text { ani }}\left(\boldsymbol{F}_{\boldsymbol{x}}, \boldsymbol{F}^{A^{-1}}, \boldsymbol{f}_{0}, \boldsymbol{s}_{0}\right)=\frac{a_{f}}{2 b_{f}}\left(e^{b_{f}\left\langle I_{4, f_{0}}^{E}-1\right\rangle^{2}}-1\right)+\frac{a_{s}}{2 b_{s}}\left(e^{b_{s}\left\langle I_{4, s_{0}}^{E}-1\right\rangle^{2}}-1\right)+\frac{a_{f s}}{2 b_{f s}}\left(e^{b_{f s} I_{8, f_{0} s_{0}}^{E}}-1\right),
\end{array}
$$

with

$$
\begin{array}{rlrl}
I I_{\hat{\boldsymbol{F}}_{x}}^{E} & =J_{\boldsymbol{x}}^{-2 / 3} J^{A^{2 / 3}}\left(\boldsymbol{F}_{\boldsymbol{x}} \boldsymbol{F}^{A}\right):\left(\boldsymbol{F}_{\boldsymbol{x}} \boldsymbol{F}^{A^{-1}}\right) ; & I_{4, f_{0}}^{E}=\left(\boldsymbol{F}_{\boldsymbol{x}} \boldsymbol{F}^{A^{-1}} \boldsymbol{f}_{0}\right) \cdot\left(\boldsymbol{F}_{\boldsymbol{x}} \boldsymbol{F}^{A^{-1}} \boldsymbol{f}_{0}\right) ; \\
I_{4, \boldsymbol{s}_{0}}^{E}=\left(\boldsymbol{F}_{\boldsymbol{x}} \boldsymbol{F}^{A^{-1}} \boldsymbol{s}_{0}\right) \cdot\left(\boldsymbol{F}_{\boldsymbol{x}} \boldsymbol{F}^{A^{-1}} \boldsymbol{s}_{0}\right) ; & I_{8, \boldsymbol{f}_{0} \boldsymbol{s}_{0}}^{E}=\left(\boldsymbol{F}_{\boldsymbol{x}} \boldsymbol{F}^{A^{-1}} \boldsymbol{f}_{0}\right) \cdot\left(\boldsymbol{F}_{\boldsymbol{x}} \boldsymbol{F}^{A^{-1}} \boldsymbol{s}_{0}\right) .
\end{array}
$$

For the specific constitutive model defined in (18), the elements of the set $\left\{\partial_{\boldsymbol{F}_{\boldsymbol{x}}} W, \partial_{\boldsymbol{H}_{\boldsymbol{x}}} W, \partial_{J_{\boldsymbol{x}}} W\right\}$ featuring in the definition of $\boldsymbol{P}$ in (36) are

$$
\begin{aligned}
\partial_{\boldsymbol{F}_{\boldsymbol{x}}} W^{E} & =a e^{b\left(I I_{\hat{\boldsymbol{F}}_{x}}^{E}-3\right)} J_{\boldsymbol{x}}^{-\frac{2}{3}} J^{A^{\frac{2}{3}}}\left(\boldsymbol{F}_{\boldsymbol{x}} \boldsymbol{F}^{A^{-1}} \boldsymbol{F}^{A^{-T}}\right)+2 a_{f} e^{b_{f}\left\langle I_{4, f_{0}}^{E}-1\right)^{2}}\left\langle I_{4, f_{0}}-1\right\rangle\left(\boldsymbol{F}_{\boldsymbol{x}} \boldsymbol{F}^{A^{-1}} \boldsymbol{f}_{0} \otimes \boldsymbol{F}^{A^{-1}} \boldsymbol{f}_{0}\right) \\
& +2 a_{s} e^{b_{s}\left(I_{4, s_{0}}^{E}-1\right)^{2}}\left\langle I_{4, s_{0}}^{E}-1\right\rangle\left(\boldsymbol{F}_{\boldsymbol{x}} \boldsymbol{F}^{A^{-1}} \boldsymbol{s}_{0} \otimes \boldsymbol{F}^{A^{-1}} \boldsymbol{s}_{0}\right) \\
& +a_{f s} e^{b_{f s} I_{8, f_{0} s_{0}}^{E}} I_{8, f_{0} \boldsymbol{s}_{0}}^{E}\left(\boldsymbol{F}_{\boldsymbol{x}} \boldsymbol{F}^{A^{-1}} \boldsymbol{f}_{0} \otimes \boldsymbol{s}_{0}+\boldsymbol{F}_{\boldsymbol{x}} \boldsymbol{F}^{A^{-1}} \boldsymbol{s}_{0} \otimes \boldsymbol{f}_{0}\right) ; \\
\partial_{\boldsymbol{H}_{\boldsymbol{x}}} W^{E} & =\mathbf{0} \\
\partial_{J_{\boldsymbol{x}}} W^{E} & =-\frac{a}{3} J_{\boldsymbol{x}}^{-1} I I_{\hat{\boldsymbol{F}}_{x}}^{E} e^{b\left(I I_{\hat{\boldsymbol{F}}_{x}}^{E}-3\right)}+J^{A^{-1}} U^{\prime}\left(J_{\boldsymbol{x}}^{-1}\right) .
\end{aligned}
$$


Remark 3. The isotropic invariant isochoric contribution $\hat{W}$ in (18) has been replaced in (39) by its elastic counterpart $\hat{W}^{E}$. Notice that $\hat{W}^{E}$ depends upon invariant $I I_{\hat{\boldsymbol{F}}_{x}}^{E}$ in (40), which can be written as

$$
I I_{\hat{\boldsymbol{F}}_{x}}^{E}=J_{\boldsymbol{x}}^{-2 / 3} J^{A^{2 / 3}}\left(\boldsymbol{F}_{\boldsymbol{x}} \boldsymbol{F}^{A^{-1}}\right):\left(\boldsymbol{F}_{\boldsymbol{x}} \boldsymbol{F}^{A^{-1}}\right)=J_{\boldsymbol{x}}^{-2 / 3} J^{A^{2 / 3}}\left(\boldsymbol{C}^{A^{-1}}: \boldsymbol{F}_{\boldsymbol{x}}^{T} \boldsymbol{F}_{\boldsymbol{x}}\right),
$$

with $\boldsymbol{C}^{A^{-1}}=\boldsymbol{F}^{A^{-1}} \boldsymbol{F}^{A^{-T}}$. In this case, $\boldsymbol{C}^{A^{-1}}$ plays a similar role to that of a structured tensor used to embed anisotropy.

\subsection{Electrophysiology of the heart}

The objective of this section is to briefly describe the phenomenological aspects underlying the governing equation (4). Specifically, the definition of the flux vector $\boldsymbol{Q}$ and the source term $f_{\phi}$ will be presented. In analogy with the Fick's Law, it is customary [7,46-49,51,53,62] to relate $\boldsymbol{Q}$ with the material gradient of the transmembrane potential $\phi$ as

$$
Q=D \nabla_{0} \phi
$$

where $\boldsymbol{D}$ represents the second order conductivity tensor [53], defined in terms of the electrical conductivities $d_{\text {iso }}$ and $d_{\text {ani }}[7,53,62]$ as

$$
\boldsymbol{D}=d_{\text {iso }} J_{\boldsymbol{x}}^{-2} \boldsymbol{H}_{\boldsymbol{x}}^{T} \boldsymbol{H}_{\boldsymbol{x}}+d_{\text {ani }} \boldsymbol{f}_{0} \otimes \boldsymbol{f}_{0} .
$$

The source term $f_{\phi}$ depends on the transmembrane potential $\phi$, gating variables ${ }^{5}$ and ion concentrations [32,41-43]. Both sets of (scalar) gate and ion concentration variables are represented by the vector $\boldsymbol{q} \in \mathbb{R}^{n_{\boldsymbol{q}}}$, with $n_{\boldsymbol{q}}$ the total number of gate numbers and ion concentrations. The evolution equations for $\boldsymbol{q}$ can be mathematically modelled via a set of first order differential equations [32,46-49], allowing them to be treated as internal variables, as

$$
\dot{\boldsymbol{q}}(\phi)=\boldsymbol{g}(\phi, \boldsymbol{q}(\phi)),
$$

with $g: \mathbb{R}^{n_{q}+1} \rightarrow \mathbb{R}^{n_{q}}$. It is customary to assume an additive decomposition of the source term as

$$
f_{\phi}(\phi)=\tilde{f}_{\phi}(\phi, \boldsymbol{q}(\phi)) ; \quad \tilde{f}_{\phi}(\phi, \boldsymbol{q}(\phi))=I_{\text {stim }}+I_{\text {sum }}(\phi, \boldsymbol{q}(\phi)),
$$

with $I_{\text {sum }}: \mathbb{R}^{n_{q}+1} \rightarrow \mathbb{R}$. The first term $I_{\text {stim }}$ in $(46)_{b}$ enables the initial propagation of the electrical wave (4). The choice of the ionic model dictates the form of the nonlinear term $I_{\text {sum }}$ in $(46)_{b}$. Two ionic models are considered in this paper. In the first, known as Bueno-Orovio model [49], $I_{\text {sum }}$ is defined as

$$
I_{\mathrm{sum}}=I_{\mathrm{fi}}+I_{\mathrm{si}}+I_{\mathrm{so}},
$$

where $I_{\mathrm{fi}}, I_{\mathrm{si}}$ and $I_{\mathrm{so}}$ represent three electrical currents which depend linearly on three gating variables. ${ }^{6}$ The reader is referred to [49] for the expressions of each of the current contributions of $I_{\text {sum }}$ in (47). Finally, the evolution equations for $\boldsymbol{q}(45)_{b}$, also linear in this case, can be found in [49]. The second model considered, namely the Ten Tusscher model [47,48], incorporates a more complex representation of $I_{\text {sum }}$, additively decomposed as

$$
I_{\mathrm{sum}}=I_{\mathrm{Na}}+I_{\mathrm{bNa}}+I_{\mathrm{NaK}}+I_{\mathrm{NaCa}}+I_{\mathrm{K} 1}+I_{\mathrm{Kr}}+I_{\mathrm{Ks}}+I_{\mathrm{pK}}+I_{\mathrm{t} 0}+I_{\mathrm{CaL}}+I_{\mathrm{bCa}}+I_{\mathrm{pCa}},
$$

where each electrical current is nonlinearly related to thirteen gating variables and four ion concentrations. The reader is referred to $[47,48]$ for the expressions of each of the current contributions of $I_{\text {sum }}$ in (48). In addition, the evolution equations for $\boldsymbol{q}(45)_{b}$, can be found in [48].

\subsection{Electro-mechanical activation of the heart}

This section presents the mechanism responsible for the coupling between the mechanical physics with the transmembrane potential $\phi$. Specifically, evolution equations for $T_{a}$ and $\gamma_{f_{0}}$ for both active stress and active strain approaches in Sections 4.1.2 and 4.1.3 will be presented.

5 Gate variables represent the probability of a gate in the cell membrane associated with a specific ion to be open.

6 Therefore, the vector $\boldsymbol{q}$ exclusively includes these three gating variables. 


\subsubsection{Active stress approach}

In the case of active stress, the evolution equations for $T_{a}$ can be expressed as

$$
\dot{T}_{a}=h_{T_{a}}(\phi, \boldsymbol{q}(\phi)),
$$

with $h_{T_{a}}: \mathbb{R}^{n_{\boldsymbol{q}}+1} \rightarrow \mathbb{R}$. The model proposed by Nash and Panfilov [51] considers the following expression for $h_{T_{a}}$ (49)

$$
h_{T_{a}}=\varepsilon(u)\left(k_{T_{a}} u-T_{a}\right) ; \quad \varepsilon(u)=\left\{\begin{array}{ll}
1 & \text { if } u>0 \\
10 & \text { if } u<0
\end{array} ; \quad u=\frac{(\phi+84)}{85.7}(\mathrm{mV}),\right.
$$

where $k_{T_{a}}$ is a constant with dimensions of stress [51]. Alternatively, Wong et al. [53] adapted the model in (50) to be used in conjunction with the Ten Tusscher model in (48). In this case, the evolution equation for $T_{a}$ depends on the calcium concentration $c_{\mathrm{Ca}}$ as

$$
h_{T_{a}}=\varepsilon\left(c_{\mathrm{Ca}}\right)\left(\eta\left(c_{\mathrm{Ca}}-c_{\mathrm{Ca}, 0}\right)-T_{a}\right) ; \quad \varepsilon\left(c_{\mathrm{Ca}}\right)=\varepsilon_{0}+\left(\varepsilon_{\infty}-\varepsilon_{0}\right) e^{A} ; \quad A=-e^{-\xi\left(c_{\mathrm{Ca}}-c_{\mathrm{Ca}}^{\mathrm{crit}}\right)},
$$

where $\left\{\varepsilon_{0}, \varepsilon_{\infty}, \xi, \eta\right\}$ constants and $\left\{c_{\mathrm{Ca}, 0}, c_{\mathrm{Ca}}^{\text {crit }}\right\}$, the resting and critical calcium concentrations respectively (refer to [53]).

\subsubsection{Active strain approach}

In the case of active strain, the evolution equations for $\gamma_{f_{0}}$ can be expressed as

$$
\dot{\gamma}_{f_{0}}=h_{\gamma_{f_{0}}}(\phi, \boldsymbol{q}(\phi)),
$$

with $=h_{\gamma_{f_{0}}}: \mathbb{R}^{n_{q}+1} \rightarrow \mathbb{R}$. In reference [7], Rossi et al. proposed the following thermodynamically consistent expression for the function $h_{\gamma_{f_{0}}}$ in (52)

$$
h_{\gamma_{f_{0}}}=\frac{1}{\hat{\mu}_{A} c_{\mathrm{Ca}}^{2}}\left(F_{A}+\frac{2 I_{4, f_{0}}}{\left(1+\gamma_{f_{0}}\right)^{3}}-2 I_{4, f_{0}}\right) \text {, }
$$

where $\hat{\mu}_{A}$ represents a viscous-type term, $c_{\mathrm{Ca}}$ denotes the calcium concentration and $F_{A}$ is the dimensionless active force along the fibre direction $\boldsymbol{f}_{0}$, defined as

$$
\begin{array}{rlrl}
F_{A} & =\alpha\left(c_{\mathrm{Ca}}-c_{\mathrm{Ca}, 0}\right)^{2} \chi_{\left[l_{\min }, l_{\max }\right]}(\mathcal{F}(l)) ; & \chi_{\left[l_{\min }, l_{\max }\right]}(\mathcal{F}(l))= \begin{cases}\mathcal{F}(l) & \text { if } l \in\left[l_{\min }, l_{\max }\right] \\
0 & \text { if } l \notin\left[l_{\min }, l_{\max }\right]\end{cases} \\
l=I_{4, f_{0}} l_{0} ; & \mathcal{F}(l)=\frac{d_{0}}{2}+\sum_{n=1}^{3} d_{n} \cos (n l)+e_{n} \sin (n l) .
\end{array}
$$

In (54), $\alpha$ represents the active force of a sarcomere, $c_{\mathrm{Ca}, 0}$, the resting calcium concentration, $\left\{l_{\min }, l_{\max }\right\}$, the minimum and maximum measured sarcomere lengths and $\left\{d_{0}, d_{n}, e_{n}\right\}$, constants calibrated to match the experimental data reported in reference [72].

Remark 4. Notice in Eqs. (53) and (54) $)_{a}$ that both $\dot{\gamma}_{f_{0}}$ and $F_{A}$ depend on the calcium concentration $c_{\mathrm{Ca}}$. In principle, this precludes the use of the Bueno-Orovio model in (47) as this model does not incorporate ionic concentrations as part of the internal variables $\boldsymbol{q}$ (45). In order to circumvent this drawback, some authors [7] use a simplified approach where the calcium concentration $c_{\mathrm{Ca}}$ is assumed to be equivalent to the internal variable $s$ in [49]. This simplification allows the use of the Bueno-Orovio model in conjunction with the active strain approach.

\section{Variational formulation for cardiac electro-mechanics}

\subsection{Standard three-field $\boldsymbol{x}$ - $\phi$ - $p$ formulation}

In this section, the weak forms associated with the governing equations in Section 3 will be presented for the case of truly incompressibility. These include the conservation of linear momentum equation (3), denoted as $\mathcal{W}_{\boldsymbol{x}}$; the monodomain model for the evolution of the transmembrane potential (4), denoted as $\mathcal{W}_{\phi}$; and the incompressibility 
constraint (i.e. $J=1$ ), denoted as $\mathcal{W}_{p}$. In this formulation, the unknown fields are $\{\boldsymbol{x}, \phi, p\} \in \mathbb{V}^{x} \times \mathbb{V}^{\phi} \times \mathbb{V}^{p}$, as

$$
\begin{aligned}
& \mathbb{V}^{x}=\left\{\boldsymbol{x}: \Omega_{0} \rightarrow \mathbb{R}^{3} ;\right.\left.(\boldsymbol{x})_{i} \in H^{1}\left(\Omega_{0}\right)\right\} ; \\
& \mathbb{V}^{\phi}=\left\{\phi: \Omega_{0} \rightarrow \mathbb{R} ; \quad \phi \in H^{1}\left(\Omega_{0}\right)\right\} ; \\
& \mathbb{V}^{p}=\left\{p: \Omega_{0} \rightarrow \mathbb{R} ; \quad p \in \mathbb{L}_{2}\left(\Omega_{0}\right)\right\},
\end{aligned}
$$

where $p$ represents the Lagrange multiplier field for the weak enforcement of the incompressibility constraint. Let the virtual variations of $\{\boldsymbol{x}, \phi, p\}$ be defined as $\{\delta \boldsymbol{x}, \delta \phi, \delta p\} \in \mathbb{V}_{0}^{\boldsymbol{x}} \times \mathbb{V}^{\phi} \times \mathbb{V}^{p}$, with

$$
\mathbb{V}_{0}^{x}=\left\{x \in \mathbb{V}^{x}, \quad x=\mathbf{0} \text { on } \partial_{x} \Omega_{0}\right\}
$$

The weak forms $\left\{\mathcal{W}_{\boldsymbol{x}}, \mathcal{W}_{\phi}, \mathcal{W}_{p}\right\}$ can then be written as

$$
\begin{aligned}
\mathcal{W}_{\boldsymbol{x}}(\boldsymbol{x}, \phi, p) & =\int_{\Omega_{0}}\left(\boldsymbol{P}+p \boldsymbol{H}_{\boldsymbol{x}}\right): \nabla_{0} \delta \boldsymbol{x} d \Omega_{0}-\int_{\Omega_{0}} \delta \boldsymbol{x} \cdot \boldsymbol{b}_{0} d \Omega_{0}-\int_{\partial \Omega_{0}} \delta \boldsymbol{x} \cdot \boldsymbol{t}_{0} d \Gamma=0 ; \\
\mathcal{W}_{\phi}(\boldsymbol{x}, \phi) & =\int_{\Omega_{0}} \delta \phi \frac{\partial \phi}{\partial t} d \Omega_{0}+\int_{\Omega_{0}} \nabla_{0} \delta \phi \cdot \boldsymbol{Q} d \Omega_{0}-\int_{\Omega_{0}} \delta \phi f_{\phi} d \Omega_{0}=0 ; \\
\mathcal{W}_{p}(\boldsymbol{x}) & =\int_{\Omega_{0}} \delta p\left(J_{\boldsymbol{x}}-1\right) d \Omega_{0}=0,
\end{aligned}
$$

with $\boldsymbol{P}$ as in (23) for active stress or as in (10) for active strain and with $\boldsymbol{Q}$ defined as in (43)-(44).

\subsection{New mixed formulations for cardiac electro-mechanics}

Following the work by the authors in $[30,58,73,74]$ in the context of nonlinear elasticity and electro-elasticity, new tailor-made mixed variational formulations will be presented for cardiac electro-mechanics.

\subsubsection{Mixed formulation for active stress approach}

A new mixed formulation is presented for the active stress approach, named MFA- $T_{a}$. In this formulation, the unknown fields are $\{\boldsymbol{x}, \phi, p\} \in \mathbb{V}^{x} \times \mathbb{V}^{\phi} \times \mathbb{V}^{p}$ (as in the three-field formulation in Section 5.1) and an additional set of fields denoted as $\{\boldsymbol{F}, \boldsymbol{H}, J, \boldsymbol{A}\} \in \mathbb{V}^{\boldsymbol{F}} \times \mathbb{V}^{\boldsymbol{H}} \times \mathbb{V}^{J} \times \mathbb{V}^{\boldsymbol{A}}$ and their work conjugates $\left\{\boldsymbol{\Sigma}_{\boldsymbol{F}}, \boldsymbol{\Sigma}_{\boldsymbol{H}}, \Sigma_{J}, \boldsymbol{\Sigma}_{\boldsymbol{A}}\right\} \in$ $\mathbb{V}^{F} \times \mathbb{V}^{H} \times \mathbb{V}^{J} \times \mathbb{V}^{A}$, with

$$
\begin{aligned}
& \mathbb{V}^{\boldsymbol{F}}=\left\{\boldsymbol{F}: \Omega_{0} \rightarrow \mathbb{R}^{3 \times 3} ;\right.\left.(\boldsymbol{F})_{i I} \in \mathbb{L}_{2}\left(\Omega_{0}\right)\right\} ; \\
& \mathbb{V}^{\boldsymbol{H}}=\left\{\boldsymbol{H}: \Omega_{0} \rightarrow \mathbb{R}^{3 \times 3} ;\right.\left.(\boldsymbol{H})_{i I} \in \mathbb{L}_{2}\left(\Omega_{0}\right)\right\} ; \\
& \mathbb{V}^{J}=\left\{J: \Omega_{0} \rightarrow \mathbb{R} ; \quad J \in \mathbb{L}_{2}\left(\Omega_{0}\right)\right\} ; \\
& \mathbb{V}^{\boldsymbol{A}}=\left\{\boldsymbol{H}: \Omega_{0} \rightarrow \mathbb{R}^{3} ; \quad(\boldsymbol{A})_{I} \in \mathbb{L}_{2}\left(\Omega_{0}\right)\right\},
\end{aligned}
$$

where both set of fields are forced to converge weakly to the following set of fields

$$
\{\boldsymbol{F}, \boldsymbol{H}, J, \boldsymbol{A}\} \rightarrow\left\{\boldsymbol{F}_{\boldsymbol{x}}, \boldsymbol{H}_{\boldsymbol{x}}, J_{\boldsymbol{x}}, \boldsymbol{\nabla}_{0} \phi\right\} ; \quad\left\{\boldsymbol{\Sigma}_{\boldsymbol{F}}, \boldsymbol{\Sigma}_{\boldsymbol{H}}, \Sigma_{J}, \boldsymbol{\Sigma}_{\boldsymbol{A}}\right\} \rightarrow\left\{\partial_{\boldsymbol{F}} W, \partial_{\boldsymbol{H}} W, \partial_{J} W, \boldsymbol{D} \boldsymbol{A}\right\} .
$$

Notice that Eq. (59) $)_{a}$ represents the compatibility conditions whereas $(59)_{b}$ represents the constitutive equations. In this formulation, the weak forms $\left\{\mathcal{W}_{x}, \mathcal{W}_{\phi}, \mathcal{W}_{p}\right\}$ are expressed as

$$
\begin{aligned}
\mathcal{W}_{\boldsymbol{x}}\left(\boldsymbol{x}, \boldsymbol{\phi}, p, \boldsymbol{F}, \boldsymbol{\Sigma}_{\boldsymbol{F}}, \boldsymbol{\Sigma}_{\boldsymbol{H}}, \Sigma_{J}\right) & =\int_{\Omega_{0}}\left(\boldsymbol{P}+p \boldsymbol{H}_{\boldsymbol{x}}\right): \nabla_{0} \delta \boldsymbol{x} d \Omega_{0}-\int_{\Omega_{0}} \delta \boldsymbol{x} \cdot \boldsymbol{b}_{0} d \Omega_{0}-\int_{\partial \Omega_{0}} \delta \boldsymbol{x} \cdot \boldsymbol{t}_{0} d \Gamma \\
\mathcal{W}_{\phi}\left(\phi, \boldsymbol{\Sigma}_{\boldsymbol{A}}\right) & =\int_{\Omega_{0}} \delta \phi \frac{\partial \phi}{\partial t} d \Omega_{0}+\int_{\Omega_{0}} \nabla_{0} \delta \phi \cdot \boldsymbol{\Sigma}_{\boldsymbol{A}} d \Omega_{0}-\int_{\Omega_{0}} \delta \phi f_{\phi} d \Omega_{0} \\
\mathcal{W}_{p}(\boldsymbol{x}) & =\int_{\Omega_{0}} \delta p\left(J_{\boldsymbol{x}}-1\right) d \Omega_{0},
\end{aligned}
$$


where $\boldsymbol{P}(60)_{a}$ adopts a similar expression to that in (23) but now in terms of the unknown fields $\left\{\boldsymbol{\Sigma}_{\boldsymbol{F}}, \boldsymbol{\Sigma}_{\boldsymbol{H}}, \Sigma_{J}, \boldsymbol{F}, \boldsymbol{H}\right.$, $J\}$ as

$$
\boldsymbol{P}=\boldsymbol{\Sigma}_{\boldsymbol{F}}+\boldsymbol{\Sigma}_{\boldsymbol{H}} \times \boldsymbol{F}_{\boldsymbol{x}}+\Sigma_{J} \boldsymbol{H}_{\boldsymbol{x}}+T_{a}(\phi) \boldsymbol{F} \boldsymbol{f}_{0} \otimes \boldsymbol{f}_{0} .
$$

Eight additional weak forms denoted as $\left\{\mathcal{W}_{\Sigma_{F}}, \mathcal{W}_{\Sigma_{H}}, \mathcal{W}_{\Sigma_{J}}, \mathcal{W}_{\Sigma_{A}}\right\}$ and $\left\{\mathcal{W}_{F}, \mathcal{W}_{H}, \mathcal{W}_{J}, \mathcal{W}_{F}\right\}$ enforcing Eq. (59) are part of this formulation and are defined as

$$
\begin{array}{rlrl}
\mathcal{W}_{\boldsymbol{\Sigma}_{\boldsymbol{F}}}(\boldsymbol{x}, \boldsymbol{F}) & =\int_{\Omega_{0}} \delta \boldsymbol{\Sigma}_{\boldsymbol{F}}:\left(\boldsymbol{F}_{x}-\boldsymbol{F}\right) d \Omega_{0} ; & & \mathcal{W}_{\boldsymbol{F}}\left(\boldsymbol{F}, \boldsymbol{H}, J, \boldsymbol{\Sigma}_{\boldsymbol{F}}\right)=\int_{\Omega_{0}} \delta \boldsymbol{F}:\left(\partial_{\boldsymbol{F}} W-\boldsymbol{\Sigma}_{\boldsymbol{F}}\right) d \Omega_{0} ; \\
\mathcal{W}_{\boldsymbol{\Sigma}_{\boldsymbol{H}}}(\boldsymbol{x}, \boldsymbol{H})=\int_{\Omega_{0}} \delta \boldsymbol{\Sigma}_{\boldsymbol{H}}:\left(\boldsymbol{H}_{x}-\boldsymbol{H}\right) d \Omega_{0} ; & \mathcal{W}_{\boldsymbol{H}}\left(\boldsymbol{F}, \boldsymbol{H}, J, \boldsymbol{\Sigma}_{\boldsymbol{H}}\right)=\int_{\Omega_{0}} \delta \boldsymbol{H}:\left(\partial_{\boldsymbol{H}} W-\boldsymbol{\Sigma}_{\boldsymbol{H}}\right) d \Omega_{0} ; \\
\mathcal{W}_{\Sigma_{J}}(\boldsymbol{x}, J)=\int_{\Omega_{0}} \delta \Sigma_{J}\left(J_{x}-J\right) d \Omega_{0} ; & \mathcal{W}_{J}\left(\boldsymbol{F}, \boldsymbol{H}, J, \Sigma_{J}\right)=\int_{\Omega_{0}} \delta J\left(\partial_{J} W-\Sigma_{J}\right) d \Omega_{0} ; \\
\mathcal{W}_{\boldsymbol{\Sigma}_{\boldsymbol{A}}}(\phi, \boldsymbol{A})=\int_{\Omega_{0}} \delta \boldsymbol{\Sigma}_{\boldsymbol{A}} \cdot\left(\nabla_{0} \phi-\boldsymbol{A}\right) d \Omega_{0} ; & \mathcal{W}_{\boldsymbol{A}}\left(\boldsymbol{H}, J, \boldsymbol{A}, \boldsymbol{\Sigma}_{\boldsymbol{A}}\right)=\int_{\Omega_{0}} \delta \boldsymbol{A} \cdot\left(\boldsymbol{D} \boldsymbol{A}-\boldsymbol{\Sigma}_{\boldsymbol{A}}\right) d \Omega_{0},
\end{array}
$$

where the strain energy $W$ depends on the fields $\{\boldsymbol{F}, \boldsymbol{H}, J\}$ and not on $\left\{\boldsymbol{F}_{\boldsymbol{x}}, \boldsymbol{H}_{\boldsymbol{x}}, J_{\boldsymbol{x}}\right\}$ as in (5). Moreover, $\{\delta \boldsymbol{F}, \delta \boldsymbol{H}, \delta J, \delta \boldsymbol{A}\} \in \mathbb{V}^{\boldsymbol{F}} \times \mathbb{V}^{\boldsymbol{H}} \times \mathbb{V}^{J} \times \mathbb{V}^{\boldsymbol{A}}$ and $\left\{\delta \boldsymbol{\Sigma}_{\boldsymbol{F}}, \delta \boldsymbol{\Sigma}_{\boldsymbol{H}}, \delta \Sigma_{J}, \delta \boldsymbol{\Sigma}_{\boldsymbol{A}}\right\} \in \mathbb{V}^{\boldsymbol{F}} \times \mathbb{V}^{\boldsymbol{H}} \times \mathbb{V}^{J} \times \mathbb{V}^{\boldsymbol{A}}$ in (62). Finally, $\boldsymbol{D}$ in $(62)_{i}$ adopts an equivalent expression to that in (44) in terms of $\{\boldsymbol{H}, J\}$ as

$$
\boldsymbol{D}(\boldsymbol{H}, J)=d_{\text {iso }} J^{-2} \boldsymbol{H}^{T} \boldsymbol{H}+d_{\text {ani }} \boldsymbol{f}_{0} \otimes \boldsymbol{f}_{0} .
$$

\subsubsection{Mixed formulation for active strain approach}

The mixed formulation presented in Section 5.2.1 will be adapted to the active strain approach in this section. In this formulation, named as MFA- $\gamma$, the weak forms $\mathcal{W}_{\phi}$ and $\mathcal{W}_{p}$ are completely equivalent to those in (60). However, the multiplicative nature of the deformation gradient tensor in (28) introduces a different dependence of the weak form $\mathcal{W}_{\boldsymbol{x}}$ which, on the contrary to its counterpart in (60), does not depend on the fields $\{\phi, \boldsymbol{F}\}$, i.e.

$$
\mathcal{W}_{\boldsymbol{x}}\left(\boldsymbol{x}, p, \boldsymbol{\Sigma}_{\boldsymbol{F}}, \boldsymbol{\Sigma}_{\boldsymbol{H}}, \Sigma_{J}\right)=\int_{\Omega_{0}}\left(\boldsymbol{P}+p \boldsymbol{H}_{\boldsymbol{x}}\right): \nabla_{0} \delta \boldsymbol{x} d \Omega_{0}-\int_{\Omega_{0}} \delta \boldsymbol{x} \cdot \boldsymbol{b}_{0} d \Omega_{0}-\int_{\partial \Omega_{0}} \delta \boldsymbol{x} \cdot \boldsymbol{t}_{0} d \Gamma,
$$

where $\boldsymbol{P}$ is now expressed similarly to (10) as

$$
P=\Sigma_{F}+\Sigma_{H} \times F_{x}+\Sigma_{J} H_{x} .
$$

For this formulation, the weak forms (and their dependence) associated with the compatibility equations, namely $\left\{\mathcal{W}_{\Sigma_{F}}, \mathcal{W}_{\Sigma_{H}}, \mathcal{W}_{\Sigma_{J}}, \mathcal{W}_{\Sigma_{A}}\right\}$, are equivalent to those in the active stress approach in (62). However, the multiplicative nature of the deformation gradient tensor in (28) introduces a different dependence of the weak forms associated with the constitutive equations, namely

$$
\begin{aligned}
\mathcal{W}_{\boldsymbol{F}}\left(\phi, \boldsymbol{F}, \boldsymbol{H}, J, \boldsymbol{\Sigma}_{\boldsymbol{F}}\right) & =\int_{\Omega_{0}} \delta \boldsymbol{F}:\left(\partial_{\boldsymbol{F}} W^{E}-\boldsymbol{\Sigma}_{\boldsymbol{F}}\right) d \Omega_{0} ; \\
\mathcal{W}_{\boldsymbol{H}}\left(\phi, \boldsymbol{F}, \boldsymbol{H}, J, \boldsymbol{\Sigma}_{\boldsymbol{H}}\right) & =\int_{\Omega_{0}} \delta \boldsymbol{H}:\left(\partial_{\boldsymbol{H}} W^{E}-\boldsymbol{\Sigma}_{\boldsymbol{H}}\right) d \Omega_{0} ; \\
\mathcal{W}_{J}\left(\phi, \boldsymbol{F}, \boldsymbol{H}, J, \Sigma_{J}\right) & =\int_{\Omega_{0}} \delta J\left(\partial_{J} W^{E}-\Sigma_{J}\right) d \Omega_{0},
\end{aligned}
$$

where $W^{E}$ depends on the fields $\left\{\boldsymbol{F}, \boldsymbol{H}, J, \boldsymbol{F}^{A}, \boldsymbol{H}^{A}, J^{A}\right\}$ and not on $\left\{\boldsymbol{F}_{\boldsymbol{x}}, \boldsymbol{H}_{\boldsymbol{x}}, J_{\boldsymbol{x}}, \boldsymbol{F}^{A}, \boldsymbol{H}^{A}, J^{A}\right\}$ as in (35). Notice in above Eq. (66) the extra dependence with respect to the field $\phi$ (due to the dependence of $W$ with respect to the active contribution of the deformation gradient tensor $\boldsymbol{F}^{A}$, see Eq. (28)) in contrast to those in (60). 


\begin{tabular}{|c|c|}
\hline \multicolumn{2}{|c|}{ ELEM1 } \\
\hline $\boldsymbol{x}$ & $P_{2}$ \\
\hline$\phi$ & $P_{2}$ \\
\hline$p$ & $P_{0}$ \\
\hline$\left\{\boldsymbol{F}, \boldsymbol{\Sigma}_{\boldsymbol{F}}\right\}$ & $P_{1}^{D}$ \\
\hline$\left\{\boldsymbol{H}, \boldsymbol{\Sigma}_{\boldsymbol{H}}\right\}$ & $P_{1}^{D}$ \\
\hline$\left\{J, \Sigma_{J}\right\}$ & $P_{0}$ \\
\hline$\left\{\boldsymbol{A}, \boldsymbol{\Sigma}_{\boldsymbol{A}}\right\}$ & $P_{1}^{D}$ \\
\hline
\end{tabular}

\begin{tabular}{|c|c|}
\hline \multicolumn{2}{|c|}{ ELEM2 } \\
\hline $\boldsymbol{x}$ & $P_{2}^{+}$ \\
\hline$\phi$ & $P_{2}$ \\
\hline$p$ & $P_{1}^{D}$ \\
\hline$\left\{\boldsymbol{F}, \boldsymbol{\Sigma}_{\boldsymbol{F}}\right\}$ & $P_{2}^{D}$ \\
\hline$\left\{\boldsymbol{H}, \boldsymbol{\Sigma}_{\boldsymbol{H}}\right\}$ & $P_{2}^{D}$ \\
\hline$\left\{J, \Sigma_{J}\right\}$ & $P_{1}^{D}$ \\
\hline$\left\{\boldsymbol{A}, \boldsymbol{\Sigma}_{\boldsymbol{A}}\right\}$ & $P_{1}^{D}$ \\
\hline
\end{tabular}

Fig. 4. Summary of the interpolation types for each variable in the different mixed variables formulations assessed in this manuscript. The superscript + denotes the use of a Crouzeix-Raviart element type [75].

\section{Computational implementation}

\subsection{General remarks}

As standard in Finite Elements, the domain $\Omega_{0}$ described in Section 2 and representing the myocardium is subdivided into a finite of non-overlapping elements $e \in \mathbb{E}$ such that

$$
\Omega_{0} \approx \Omega_{0}^{h}=\bigcup_{e \in \mathbb{E}} \Omega_{0}^{e} .
$$

The unknown fields in the mixed formulations in Sections 5.2.1 band 5.2.2 are discretised using the following functional spaces $\{\boldsymbol{x}, \phi, p\} \in \mathbb{V}^{x^{h}} \times \mathbb{V}^{\phi^{h}} \times \mathbb{V}^{p^{h}}$ and $\{\boldsymbol{F}, \boldsymbol{H}, \boldsymbol{J}, \boldsymbol{A}\} \in \mathbb{V}^{\boldsymbol{F}^{h}} \times \mathbb{V}^{\boldsymbol{H}^{h}} \times \mathbb{V}^{J^{h}} \times \mathbb{V}^{\boldsymbol{A}^{h}}$ and $\left\{\boldsymbol{\Sigma}_{\boldsymbol{F}}, \boldsymbol{\Sigma}_{\boldsymbol{H}}, \Sigma_{J}, \boldsymbol{\Sigma}_{\boldsymbol{A}}\right\} \in$ $\mathbb{V}^{h} \times \mathbb{V}^{\boldsymbol{H}^{h}} \times \mathbb{V}^{J^{h}} \times \mathbb{V}^{\boldsymbol{A}^{h}}$, with

$$
\begin{aligned}
\mathbb{V}^{x^{h}}=\left\{\boldsymbol{x} \in \mathbb{V}^{\boldsymbol{x}} ; \quad \boldsymbol{x}=\sum_{a=1}^{n_{\boldsymbol{x}}} \boldsymbol{x}_{a} N_{a}^{\boldsymbol{x}}\right\} ; & \mathbb{V}^{\phi^{h}}=\left\{\phi^{h} \in \mathbb{V}^{\phi} ; \quad \boldsymbol{\phi}=\sum_{a=1}^{n_{\phi}} \phi_{a} N_{a}^{\phi}\right\} ; \\
\mathbb{V}^{p^{h}}=\left\{p^{h} \in \mathbb{V}^{p} ; \quad p=\sum_{a=1}^{n_{p}} p_{a} N_{a}^{p}\right\} ; & \mathbb{V}^{\boldsymbol{F}^{h}}=\left\{\boldsymbol{F}^{h} \in \mathbb{V}^{\boldsymbol{F}} ; \quad \boldsymbol{F}=\sum_{a=1}^{n_{\boldsymbol{F}}} \boldsymbol{F}_{a} N_{a}^{\boldsymbol{F}}\right\} ; \\
\mathbb{V}^{\boldsymbol{H}^{h}}=\left\{\boldsymbol{H}^{h} \in \mathbb{V}^{\boldsymbol{H}} ; \quad \boldsymbol{H}=\sum_{a=1}^{n_{\boldsymbol{H}}} \boldsymbol{H}_{a} N_{a}^{\boldsymbol{H}}\right\} ; & \mathbb{V}^{J^{h}}=\left\{J^{h} \in \mathbb{V}^{J} ; \quad J=\sum_{a=1}^{n_{J}} J_{a} N_{a}^{J}\right\} ; \\
\mathbb{V}^{\boldsymbol{A}^{h}}=\left\{\boldsymbol{A}^{h} \in \mathbb{V}^{\boldsymbol{A}} ; \quad \boldsymbol{A}=\sum_{a=1}^{n_{\boldsymbol{A}}} \boldsymbol{A}_{a} N_{a}^{\boldsymbol{A}}\right\}, &
\end{aligned}
$$

where $a$ denotes the nodes used for the interpolation of the above variables and $n_{(\bullet)}$, the number of nodes associated with the variable $(\bullet)$. The functional spaces used for the virtual (and incremental) variations of the unknown fields are $\{\delta \boldsymbol{x}, \delta \phi, \delta p\} \in \mathbb{V}_{0}^{x^{h}} \times \mathbb{V}^{\phi^{h}} \times \mathbb{V}^{p^{h}}$ and $\{\delta \boldsymbol{F}, \delta \boldsymbol{H}, \delta J, \delta \boldsymbol{A}\} \in \mathbb{V}^{\boldsymbol{F}^{h}} \times \mathbb{V}^{\boldsymbol{H}^{h}} \times \mathbb{V}^{J^{h}} \times \mathbb{V}^{\boldsymbol{A}^{h}}$ and $\left\{\delta \boldsymbol{\Sigma}_{\boldsymbol{F}}, \delta \boldsymbol{\Sigma}_{\boldsymbol{H}}, \delta \Sigma_{J}, \delta \boldsymbol{\Sigma}_{\boldsymbol{A}}\right\} \in$ $\mathbb{V}^{h} \times \mathbb{V}^{\boldsymbol{H}^{h}} \times \mathbb{V}^{J^{h}} \times \mathbb{V}^{\boldsymbol{A}^{h}}$, with

$$
\mathbb{V}_{0}^{x^{h}}=\left\{\boldsymbol{x} \in \mathbb{V}^{x^{h}} ; \quad \boldsymbol{x}=\mathbf{0} ; \text { on } \partial_{t} \Omega_{0}\right\} .
$$

In order to avoid an excessive computational cost associated with the large number of unknown fields in this mixed formulation, a piecewise discontinuous interpolation of the fields $\{\boldsymbol{F}, \boldsymbol{H}, J, \boldsymbol{A}\}$ and $\left\{\boldsymbol{\Sigma}_{\boldsymbol{F}}, \boldsymbol{\Sigma}_{\boldsymbol{H}}, \Sigma_{J}, \boldsymbol{\Sigma}_{\boldsymbol{A}}\right\}$ is carried out in this work, which enables to condense out these fields using standard static condensation procedure. Specifically, two new Finite Elements have been used in this work, denoted as ELEM1 and ELEM2 . In the first, a quadratic tetrahedral element for $\boldsymbol{x}$ and $\phi$ is used in conjunction with a discontinuous linear interpolation for the fields $\{\boldsymbol{F}, \boldsymbol{H}, \boldsymbol{A}\}$ and their work conjugates and a constant interpolation for $\left\{J, \Sigma_{J}, p\right\}$. In the second, a quadratic tetrahedral element for $\boldsymbol{x}$ and $\phi$ where only the interpolation of $\boldsymbol{x}$ is enhanced with five bubble functions in analogy to the $P_{2}^{+} P_{1}^{D}$ CrouzeixRaviart [75,76]. Additionally, a discontinuous quadratic interpolation is used for the fields $\{\boldsymbol{F}, \boldsymbol{H}\}$ and their work 
conjugates and a linear interpolation for all the remaining fields. Notice that the superscript $D$ in the definition of both elements is associated with the discontinuous nature of a particular field. Fig. 4 summarises the different Finite Element spaces employed for both elements.

\subsection{Finite element semi-discretisation}

In this section, the Finite Element implementation of the set of weak forms associated with the mixed formulation MFA- $\gamma$ in Section 5.2.2 will be presented. Standard Finite Element discretisation enables the discrete form of the weak forms $\left\{\mathcal{W}_{x}, \mathcal{W}_{\phi}, \mathcal{W}_{p}\right\}$ in (60) to be expressed as

$$
\mathcal{W}_{\boldsymbol{x}}=\sum_{a=1}^{n_{x}} \boldsymbol{R}_{\boldsymbol{x}}^{a} \cdot \delta \boldsymbol{x}^{a} ; \quad \mathcal{W}_{\phi}=\sum_{a=1}^{n_{\phi}} R_{\phi}^{a} \delta \phi^{a} ; \quad \mathcal{W}_{p}=\sum_{a=1}^{n_{p}} R_{p}^{a} \delta p^{a} ;
$$

with the associated residuals $R_{x}^{a}, R_{\phi}^{a}$ and $R_{p}^{a}$ defined as

$$
\begin{aligned}
\boldsymbol{R}_{x}^{a} & =\int_{\Omega_{0}}\left(\boldsymbol{P}+p \boldsymbol{H}_{\boldsymbol{x}}\right) \nabla_{0} N_{x}^{a} d \Omega_{0}-\int_{\Omega_{0}} N_{x}^{a} \boldsymbol{b}_{0} d \Omega_{0}-\int_{\partial \Omega_{0}} N_{x}^{a} \boldsymbol{t}_{0} d \Gamma ; \\
R_{\phi}^{a} & =\int_{\Omega_{0}} N_{\phi}^{a} \frac{\partial \phi}{\partial t} d \Omega_{0}+\int_{\Omega_{0}} \nabla_{0} N_{\phi}^{a} \cdot \boldsymbol{\Sigma}_{\boldsymbol{A}} d \Omega_{0}-\int_{\Omega_{0}} N_{\phi}^{a} f_{\phi} d \Omega_{0} ; \\
R_{p}^{a} & =\int_{\Omega_{0}} N_{p}^{a}\left(J_{x}-1\right) d \Omega_{0} .
\end{aligned}
$$

Similarly, the discrete form of the compatibility equations and the constitutive equations enables their associated residuals $\left\{\boldsymbol{R}_{\Sigma_{F}}, \boldsymbol{R}_{\Sigma_{H}}, R_{\Sigma_{J}}, \boldsymbol{R}_{\Sigma_{A}}\right\}$ and $\left\{\boldsymbol{R}_{\boldsymbol{F}}, \boldsymbol{R}_{\boldsymbol{H}}, R_{J}, \boldsymbol{R}_{A}\right\}$ to be defined as

$$
\begin{aligned}
\boldsymbol{R}_{\boldsymbol{\Sigma}_{\boldsymbol{F}}}^{a} & =\int_{\Omega_{0}} N_{\boldsymbol{F}}^{a}\left(\boldsymbol{F}_{\boldsymbol{x}}-\boldsymbol{F}\right) d \Omega_{0} ; & \boldsymbol{R}_{\boldsymbol{F}}^{a} & =\int_{\Omega_{0}} N_{\boldsymbol{F}}^{a}\left(\partial_{\boldsymbol{F}} W-\boldsymbol{\Sigma}_{\boldsymbol{F}}\right) d \Omega_{0} ; \\
\boldsymbol{R}_{\boldsymbol{\Sigma}_{\boldsymbol{H}}}^{a} & =\int_{\Omega_{0}} N_{\boldsymbol{H}}^{a}\left(\boldsymbol{H}_{\boldsymbol{x}}-\boldsymbol{H}\right) d \Omega_{0} ; & \boldsymbol{R}_{\boldsymbol{H}}^{a} & =\int_{\Omega_{0}} N_{\boldsymbol{H}}^{a}\left(\partial_{\boldsymbol{H}} W-\boldsymbol{\Sigma}_{\boldsymbol{H}}\right) d \Omega_{0} ; \\
R_{\Sigma_{J}}^{a} & =\int_{\Omega_{0}} N_{J}^{a}\left(J_{\boldsymbol{x}}-J\right) d \Omega_{0} ; & R_{J}^{a} & =\int_{\Omega_{0}} N_{J}^{a}\left(\partial_{J} W-\Sigma_{J}\right) d \Omega_{0} ; \\
\boldsymbol{R}_{\boldsymbol{\Sigma}_{\boldsymbol{A}}}^{a} & =\int_{\Omega_{0}} N_{\boldsymbol{A}}^{a}\left(\nabla_{0} \phi-\boldsymbol{A}\right) d \Omega_{0} ; & \boldsymbol{R}_{\boldsymbol{A}}^{a} & =\int_{\Omega_{0}} N_{\boldsymbol{A}}^{a}\left(\boldsymbol{D} \boldsymbol{A}-\boldsymbol{\Sigma}_{\boldsymbol{A}}\right) d \Omega_{0} .
\end{aligned}
$$

Let us denote $\mathcal{U}=\left\{\boldsymbol{x}, \phi, p, \mathcal{Y}, \boldsymbol{\Sigma}_{\mathcal{Y}}\right\}$, with $\mathcal{Y}=\{\boldsymbol{F}, \boldsymbol{H}, J, \boldsymbol{A}\}$ and $\boldsymbol{\Sigma}_{\mathcal{Y}}=\left\{\boldsymbol{\Sigma}_{\boldsymbol{F}}, \boldsymbol{\Sigma}_{\boldsymbol{H}}, \Sigma_{J}, \boldsymbol{\Sigma}_{\boldsymbol{A}}\right\}$. Consistent linearisation of the above residuals with respect to the incremental variations $\Delta \mathcal{U}$ leads to the following NewtonRaphson-based update algorithm

$$
\boldsymbol{K}\left(\mathcal{U}_{k}\right) \Delta \mathcal{U}=-\mathcal{R}\left(\mathcal{U}_{k}\right) ; \quad \mathcal{U}_{k+1}=\mathcal{U}_{k}+\Delta \mathcal{U}
$$

where the stiffness matrix $\boldsymbol{K}$, emerging as a result of the consistent linearisation of the residuals in Eqs. (71) and (72), before static condensation is carried out can be expressed as

$$
\boldsymbol{K}=\left[\begin{array}{ccccc}
\boldsymbol{K}_{x \boldsymbol{x}} & \mathbf{0} & \boldsymbol{K}_{\boldsymbol{x} p} & \mathbf{0} & \boldsymbol{K}_{\boldsymbol{x} \Sigma_{\mathcal{Y}}} \\
\mathbf{0} & \boldsymbol{K}_{\phi \phi} & \mathbf{0} & \mathbf{0} & \boldsymbol{K}_{\phi \Sigma_{\mathcal{Y}}} \\
\boldsymbol{K}_{\boldsymbol{x} p}^{T} & \mathbf{0} & \mathbf{0} & \mathbf{0} & \mathbf{0} \\
\mathbf{0} & \boldsymbol{K}_{\mathcal{Y} \phi} & \mathbf{0} & \boldsymbol{K}_{\mathcal{Y} \mathcal{Y}} & \boldsymbol{K}_{\mathcal{Y} \Sigma_{\mathcal{Y}}} \\
\boldsymbol{K}_{\boldsymbol{x} \Sigma_{\mathcal{Y}}}^{T} & \boldsymbol{K}_{\boldsymbol{\phi} \Sigma_{\mathcal{Y}}}^{T} & \mathbf{0} & \boldsymbol{K}_{\mathcal{Y} \Sigma_{\mathcal{Y}}}^{T} & \mathbf{0}
\end{array}\right] .
$$

The expression of each of the components of $\boldsymbol{K}$ in above Eq. (74) will be presented in the following derivations. As shown in reference [60], the stiffness matrix $\boldsymbol{K}_{\boldsymbol{x} x}$, corresponding to the linearisation of $\boldsymbol{R}_{\boldsymbol{x}}$ in $(71)_{a}$ with respect to 
$\Delta \boldsymbol{x}$ is obtained as

$$
\boldsymbol{K}_{\boldsymbol{x} \boldsymbol{x}}^{a b}=\mathcal{E}: \int_{\Omega_{0}}\left(\boldsymbol{\Sigma}_{\boldsymbol{H}}+\left(\Sigma_{\boldsymbol{J}}+p\right) \boldsymbol{F}_{\boldsymbol{x}}\right)\left(\nabla_{0} N_{\boldsymbol{x}}^{a} \times \nabla_{0} N_{\boldsymbol{x}}^{b}\right) d \Omega_{0} .
$$

In addition, the linearisation of $\boldsymbol{R}_{\boldsymbol{x}}$ with respect to $\Delta p$ leads to the stiffness matrix contribution $\boldsymbol{K}_{\boldsymbol{x} p}$, obtained as

$$
\boldsymbol{K}_{\boldsymbol{x} p}^{a b}=\int_{\Omega_{0}} N_{p}^{b}\left(\boldsymbol{H}_{\boldsymbol{x}} \nabla_{0} N_{\boldsymbol{x}}^{a}\right) d \Omega_{0} .
$$

Moreover, the linearisation of this residual, namely $\boldsymbol{R}_{\boldsymbol{x}}$, with respect to the set $\boldsymbol{\Sigma}_{\mathcal{Y}}$ leads to the stiffness contribution $\boldsymbol{K}_{\boldsymbol{x} \boldsymbol{\Sigma}_{\boldsymbol{Y}}}$ defined as

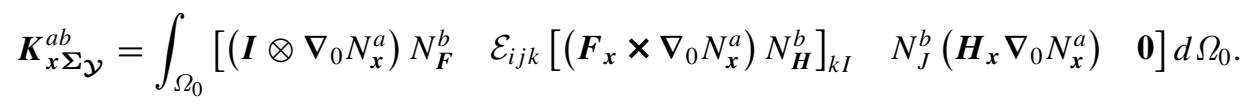

The linearisation of the residual $\boldsymbol{R}_{\phi}$ in Eq. $(71)_{b}$ leads to the stiffness contribution $\boldsymbol{K}_{\phi \phi}$. In the case of using a backward-Euler time integrator for the (time dependent) first term on the right hand side of this equation, $\boldsymbol{K}_{\phi \phi}$ can be obtained as

$$
\boldsymbol{K}_{\phi \phi}^{a b}=\int_{\Omega_{0}} N_{\phi}^{a} N_{\phi}^{b} \frac{1}{\Delta t} d \Omega_{0}+\int_{\Omega_{0}} N_{\phi}^{a} N_{\phi}^{b} \frac{D f_{\phi}}{D \phi} d \Omega_{0},
$$

where the derivation of $\frac{D f_{\phi}}{D \phi}$ can be found in Remark 4. In addition, the linearisation of residual $\boldsymbol{R}_{\phi}$ respect to the work conjugate $\Sigma_{\boldsymbol{A}}$ leads to the following expression

$$
\boldsymbol{K}_{\phi \Sigma_{\boldsymbol{A}}}^{a b}=\int_{\Omega_{0}} \nabla_{0} N_{\phi}^{a} N_{A}^{b} d \Omega_{0} .
$$

Linearisation of the residual $\boldsymbol{R}_{\mathcal{Y}}$ with respect to $\boldsymbol{\Sigma}_{\mathcal{Y}}$ leads to the stiffness $\boldsymbol{K}_{\mathcal{Y} \boldsymbol{\Sigma}_{\mathcal{Y}}}$, defined as

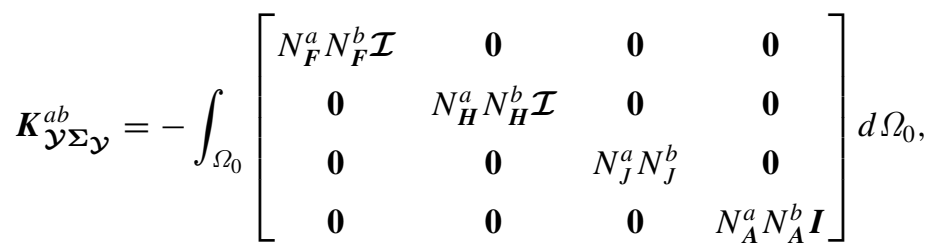

where $\mathcal{I}$ represents the fourth order identity tensor $\mathcal{I}_{i I j J}=\delta_{i j} \delta_{I J}$ and $\boldsymbol{I}$, the second order identity tensor. Linearisation of the residual $\boldsymbol{R}_{\mathcal{Y}}$ with respect to $\mathcal{Y}$ leads to the stiffness $\boldsymbol{K}_{\mathcal{Y} \mathcal{Y}}$, defined as

$$
\boldsymbol{K}_{\mathcal{Y} \mathcal{Y}}^{a b}=-\int_{\Omega_{0}}\left[\begin{array}{cccc}
N_{\boldsymbol{F}}^{a} N_{\boldsymbol{F}}^{b} W_{\boldsymbol{F} \boldsymbol{F}} & N_{\boldsymbol{F}}^{a} N_{\boldsymbol{H}}^{b} W_{\boldsymbol{F} \boldsymbol{H}} & N_{\boldsymbol{F}}^{a} N_{J}^{b} W_{\boldsymbol{F} J} & \mathbf{0} \\
N_{\boldsymbol{H}}^{a} N_{\boldsymbol{F}}^{b} W_{\boldsymbol{H} \boldsymbol{F}} & N_{\boldsymbol{H}}^{a} N_{\boldsymbol{H}}^{b} W_{\boldsymbol{H} \boldsymbol{H}} & N_{\boldsymbol{H}}^{a} N_{J}^{b} W_{\boldsymbol{H} J} & \mathbf{0} \\
N_{J}^{a} N_{\boldsymbol{F}}^{b} W_{J \boldsymbol{F}} & N_{J}^{a} N_{\boldsymbol{H}}^{b} W_{J \boldsymbol{H}} & N_{J}^{a} N_{J}^{b} W_{J J} & \mathbf{0} \\
\mathbf{0} & \boldsymbol{k}_{\boldsymbol{A} \boldsymbol{H}} & \boldsymbol{k}_{\boldsymbol{A} J} & N_{\boldsymbol{A}}^{a} N_{\boldsymbol{A}}^{b} \boldsymbol{Q}
\end{array}\right] d \Omega_{0},
$$

where $W_{\boldsymbol{B} \boldsymbol{C}}=\frac{\partial^{2} W}{\partial \boldsymbol{B} \partial \boldsymbol{C}}$ with $\boldsymbol{B}$ and $\boldsymbol{C}$ any of the fields of the set $\{\boldsymbol{F}, \boldsymbol{H}, J\}$. Moreover, $\boldsymbol{k}_{\boldsymbol{A} \boldsymbol{H}}$ and $\boldsymbol{k}_{\boldsymbol{A} J}$ in above equation are obtained as

$$
\left[\boldsymbol{k}_{\boldsymbol{A} \boldsymbol{H}}\right]_{I j J}=d_{\mathrm{iso}} J^{-2} N_{\boldsymbol{A}}^{a} N_{\boldsymbol{H}}^{b}\left(H_{j I} A_{J}+\delta_{I J}[\boldsymbol{H} \boldsymbol{A}]_{j}\right) ; \quad \boldsymbol{k}_{\boldsymbol{A} J}=-2 d_{\mathrm{iso}} J^{-3} N_{\boldsymbol{A}}^{a} N_{J}^{b}\left(\boldsymbol{H}^{T} \boldsymbol{H} \boldsymbol{A}\right) .
$$

Finally, the non-vanishing $\left(\boldsymbol{K}_{\boldsymbol{A} \phi}=\mathbf{0}\right)$ contributions of the stiffness $\boldsymbol{K}_{\mathcal{Y}_{\phi}}$ are obtained as

$$
\boldsymbol{K}_{\boldsymbol{F} \phi}^{a b}=\int_{\Omega_{0}} N_{\boldsymbol{F}}^{a} N_{\phi}^{b} W_{\boldsymbol{F} \boldsymbol{F}^{A}}: \boldsymbol{U} d \Omega_{0} ; \quad \boldsymbol{K}_{\boldsymbol{H} \phi}^{a b}=\int_{\Omega_{0}} N_{\boldsymbol{H}}^{a} N_{\phi}^{b} W_{\boldsymbol{H} \boldsymbol{F}^{A}}: \boldsymbol{U} d \Omega_{0} ; \quad \boldsymbol{K}_{J \phi}^{a b}=\int_{\Omega_{0}} N_{J}^{a} N_{\phi}^{b} W_{J \boldsymbol{F}^{A}}: \boldsymbol{U} d \Omega_{0},
$$

where the second order tensor $\boldsymbol{U}$ is expressed as

$$
\boldsymbol{U}=\frac{D \boldsymbol{F}^{A}}{D \gamma_{f_{0}}} \frac{D \gamma_{f_{0}}}{D \phi}
$$


where the derivation of $\frac{D \gamma_{f_{0}}}{D \phi}$ can be found in Remark 5 .

Remark 5. Time integration of Eq. (52) via the implicit first order in time Backward Euler scheme enables to $\gamma_{f_{0}}$ at current time step $t_{n+1}$ as

$$
\gamma_{f_{0}}\left(t_{n+1}\right)=\gamma_{f_{0}}\left(t_{n}\right)+\Delta t h_{\gamma_{f_{0}}}\left(\phi\left(t_{n+1}\right), \boldsymbol{q}\left(\phi\left(t_{n+1}\right)\right)\right),
$$

where $\Delta t$ represents the magnitude of the time step used. The total derivative of both the source term $f_{\phi}$ in (46) and $\gamma_{f_{0}}$ in (52) in (85) leads to

$$
\frac{D f_{\phi}}{D \phi}=\frac{\partial f_{\phi}}{\partial \phi}+\frac{\partial f_{\phi}}{\partial \boldsymbol{q}} \cdot \frac{\partial \boldsymbol{q}}{\partial \phi} ; \quad \frac{D \gamma_{f_{0}}}{D \phi}=\frac{\partial h_{\gamma_{f_{0}}}}{\partial \phi}+\frac{\partial h_{\gamma_{f_{0}}}}{\partial \boldsymbol{q}} \cdot \frac{\partial \boldsymbol{q}}{\partial \phi} .
$$

The only field that remains to be determined in (86) is $\frac{\partial \boldsymbol{q}}{\partial \phi}$. Time integration of (45) via the implicit first order in time Backward Euler scheme enables to obtain $\boldsymbol{q}$ at $t_{n+1}$ as

$$
\boldsymbol{q}\left(t_{n+1}\right)=\boldsymbol{q}\left(t_{n}\right)+\Delta t \boldsymbol{g}\left(\phi\left(t_{n+1}\right), \boldsymbol{q}\left(t_{n+1}\right)\right) .
$$

Differentiation with respect to $\phi$ in (87) enables to obtain $\frac{\partial \boldsymbol{q}}{\partial \phi}$ as

$$
\frac{\partial \boldsymbol{q}}{\partial \phi}=\Delta t\left(\frac{\partial \boldsymbol{g}}{\partial \phi}+\frac{\partial \boldsymbol{g}}{\partial \boldsymbol{q}} \frac{\partial \boldsymbol{q}}{\partial \phi}\right) \rightarrow \frac{\partial \boldsymbol{q}}{\partial \phi}=\Delta t\left(\boldsymbol{I}_{n_{\boldsymbol{q}} \times n_{\boldsymbol{q}}}-\frac{\partial \boldsymbol{g}}{\partial \boldsymbol{q}}\right)^{-1} \frac{\partial \boldsymbol{g}}{\partial \phi} .
$$

\subsubsection{Static condensation}

In order to use the static condensation procedure for a given element $e$, the system of equations can be expressed as

$$
\left[\begin{array}{ccccc}
\boldsymbol{K}_{\boldsymbol{x} x}^{e} & \mathbf{0} & \boldsymbol{K}_{\boldsymbol{x} p}^{e} & \mathbf{0} & \boldsymbol{K}_{\boldsymbol{x} \boldsymbol{\Sigma}_{\mathcal{Y}}}^{e} \\
\mathbf{0} & \boldsymbol{K}_{\phi \phi}^{e} & \mathbf{0} & \mathbf{0} & \boldsymbol{K}_{\phi \boldsymbol{\Sigma}_{\mathcal{Y}}}^{e} \\
{\left[\boldsymbol{K}_{\boldsymbol{x} p}^{e}\right]^{T}} & \mathbf{0} & \mathbf{0} & \mathbf{0} & \mathbf{0} \\
\mathbf{0} & \boldsymbol{K}_{\mathcal{Y} \phi}^{e} & \mathbf{0} & \boldsymbol{K}_{\mathcal{Y} \mathcal{Y}}^{e} & \boldsymbol{K}_{\mathcal{Y} \Sigma_{\mathcal{Y}}^{e}}^{e} \\
{\left[\boldsymbol{K}_{\boldsymbol{x} \Sigma_{\mathcal{Y}}^{e}}^{e}\right]^{T}} & \boldsymbol{K}_{\boldsymbol{\Sigma}_{\mathcal{Y} \phi}}^{e} & \mathbf{0} & {\left[\boldsymbol{K}_{\mathcal{Y} \Sigma_{\mathcal{Y}}^{e}}^{e}\right]^{T}} & \mathbf{0}
\end{array}\right]\left[\begin{array}{c}
\Delta \boldsymbol{x}^{e} \\
\Delta \phi^{e} \\
\Delta p^{e} \\
\Delta \mathcal{Y}^{e} \\
\Delta \boldsymbol{\Sigma}_{\mathcal{Y}}^{e}
\end{array}\right]=-\left[\begin{array}{c}
\boldsymbol{R}_{\boldsymbol{x}}^{e} \\
\boldsymbol{R}_{\phi}^{e} \\
\boldsymbol{R}_{p}^{e} \\
\boldsymbol{R}_{\mathcal{Y}}^{e} \\
\boldsymbol{R}_{\Sigma_{\mathcal{Y}}^{e}}^{e}
\end{array}\right] .
$$

Replacing the values of $\Delta \mathcal{Y}^{e}$ and $\Delta \boldsymbol{\Sigma}_{\mathcal{Y}}^{e}$ from the fourth and fifth rows in above Eq. (89), enables to rewrite the element system of equations as

$$
\left[\begin{array}{ccc}
\overline{\boldsymbol{K}}_{\boldsymbol{x} x}^{e} & \overline{\boldsymbol{K}}_{\boldsymbol{x} \phi}^{e} & \boldsymbol{K}_{\boldsymbol{x} p}^{e} \\
\overline{\boldsymbol{K}}_{\phi \boldsymbol{x}}^{e} & \overline{\boldsymbol{K}}_{\phi \phi}^{e} & \mathbf{0} \\
{\left[\boldsymbol{K}_{\boldsymbol{x} p}^{e}\right]^{T}} & \mathbf{0} & \mathbf{0}
\end{array}\right]\left[\begin{array}{c}
\Delta \boldsymbol{x}^{e} \\
\Delta \phi^{e} \\
\Delta p^{e}
\end{array}\right]=-\left[\begin{array}{c}
\overline{\boldsymbol{R}}_{x}^{e} \\
\overline{\boldsymbol{R}}_{\phi}^{e} \\
\boldsymbol{R}_{p}^{e}
\end{array}\right] .
$$

where the modified matrices $\overline{\boldsymbol{K}}_{x \boldsymbol{x}}^{e}, \overline{\boldsymbol{K}}_{\boldsymbol{x} \phi}^{e}, \overline{\boldsymbol{K}}_{\phi x}^{e}, \overline{\boldsymbol{K}}_{\phi \phi}^{e}, \overline{\boldsymbol{R}}_{\boldsymbol{x}}^{e}$ and $\overline{\boldsymbol{R}}_{\phi}^{e}$ are defined as

$$
\begin{aligned}
& \overline{\boldsymbol{K}}_{\boldsymbol{x} \boldsymbol{x}}^{e}=\boldsymbol{K}_{\boldsymbol{x} \boldsymbol{\Sigma}_{\mathcal{Y}}}^{e}\left[\boldsymbol{K}_{\mathcal{Y} \Sigma_{\mathcal{Y}}}^{e}\right]^{-1} \boldsymbol{K}_{\mathcal{Y} \mathcal{Y}}^{e}\left[\boldsymbol{K}_{\mathcal{Y} \Sigma_{\mathcal{Y}}^{e}}\right]^{-T}\left[\boldsymbol{K}_{x \Sigma_{\mathcal{Y}}}^{e}\right]^{T}+\boldsymbol{K}_{\boldsymbol{x} \boldsymbol{x}}^{e} \\
& \overline{\boldsymbol{K}}_{\boldsymbol{x} \phi}^{e}=\boldsymbol{K}_{\boldsymbol{x} \boldsymbol{\Sigma} \mathcal{Y}}^{e}\left[\boldsymbol{K}_{\mathcal{Y} \boldsymbol{\Sigma}_{\mathcal{Y}}}^{e}\right]^{-1}\left(\boldsymbol{K}_{\mathcal{Y} \mathcal{Y}}^{e}\left[\boldsymbol{K}_{\mathcal{Y} \boldsymbol{\Sigma}_{\mathcal{Y}}^{e}}\right]^{-T} \boldsymbol{K}_{\boldsymbol{\Sigma}_{\mathcal{Y} \phi}^{e}}^{e}-\boldsymbol{K}_{\mathcal{Y} \phi}^{e}\right) \\
& \overline{\boldsymbol{K}}_{\phi \boldsymbol{x}}^{e}=\boldsymbol{K}_{\phi \boldsymbol{\Sigma} \mathcal{Y}}^{e}\left[\boldsymbol{K}_{\mathcal{Y} \Sigma_{\mathcal{Y}}}^{e}\right]^{-1} \boldsymbol{K}_{\mathcal{Y} \mathcal{Y}}^{e}\left[\boldsymbol{K}_{\mathcal{Y} \Sigma_{\mathcal{Y}}^{e}}\right]^{-T}\left[\boldsymbol{K}_{x \boldsymbol{\Sigma}}^{e}\right]^{T} \\
& \overline{\boldsymbol{K}}_{\phi \phi}^{e}=\boldsymbol{K}_{\phi \boldsymbol{\Sigma}_{\mathcal{Y}}}^{e}\left[\boldsymbol{K}_{\mathcal{Y} \Sigma_{\mathcal{Y}}}^{e}\right]^{-1}\left(\boldsymbol{K}_{\mathcal{Y} \mathcal{Y}}^{e}\left[\boldsymbol{K}_{\mathcal{Y} \Sigma_{\mathcal{Y}}}^{e}\right]^{-T} \boldsymbol{K}_{\boldsymbol{\Sigma}_{\mathcal{Y} \phi}}^{e}-\boldsymbol{K}_{\mathcal{Y} \phi}^{e}\right)+\boldsymbol{K}_{\phi \phi}^{e} \\
& \overline{\boldsymbol{R}}_{\boldsymbol{x}}^{e}=\boldsymbol{K}_{x \boldsymbol{\Sigma}_{\mathcal{Y}}}^{e}\left[\boldsymbol{K}_{\mathcal{Y} \Sigma_{\mathcal{Y}}}^{e}\right]^{-1}\left(\boldsymbol{K}_{\mathcal{Y} \mathcal{Y}}^{e}\left[\boldsymbol{K}_{\mathcal{Y} \Sigma_{\mathcal{Y}}^{e}}\right]^{-T} \boldsymbol{R}_{\Sigma_{\mathcal{Y}}^{e}}^{e}-\boldsymbol{R}_{\mathcal{Y}}^{e}\right)+\boldsymbol{R}_{\boldsymbol{x}}^{e} \\
& \overline{\boldsymbol{R}}_{\phi}^{e}=\boldsymbol{K}_{\phi \Sigma_{\mathcal{Y}}}^{e}\left[\boldsymbol{K}_{\mathcal{Y} \Sigma_{\mathcal{Y}}}^{e}\right]^{-1}\left(\boldsymbol{K}_{\mathcal{Y} \mathcal{Y}}^{e}\left[\boldsymbol{K}_{\mathcal{Y} \Sigma_{\mathcal{Y}}}^{e}\right]^{-T} \boldsymbol{R}_{\Sigma_{\mathcal{Y}}}^{e}-\boldsymbol{R}_{\mathcal{Y}}^{e}\right)+\boldsymbol{R}_{\phi}^{e},
\end{aligned}
$$




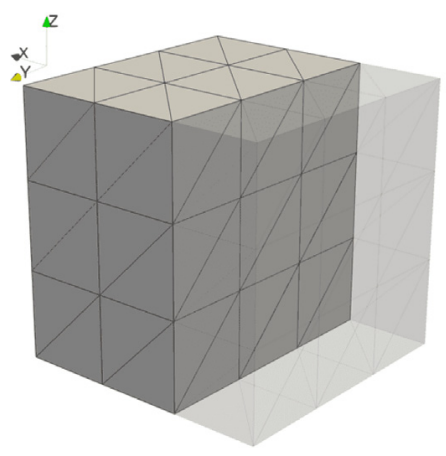

(a)

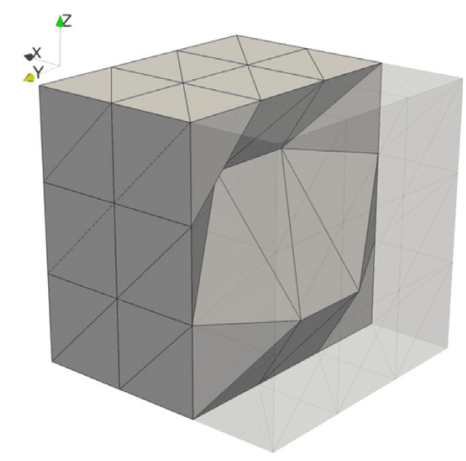

(b)

Fig. 5. Numerical example $1 .(3 \times 3 \times 3) \times 6$ tetrahedral discretisation with (a) structured and (b) unstructured mesh.

\begin{tabular}{|l|l|l|l|}
\hline \multicolumn{4}{|c|}{ Parameters for the Holzapfel-Ogden constitutive law } \\
\hline$a=0.059 \mathrm{kPa}$ & $a_{f}=0 \mathrm{kPa}$ & $a_{s}=0 \mathrm{kPa}$ & $a_{f s}=0 \mathrm{kPa}$ \\
\hline$b=8.023$ & $b_{f}=0$ & $b_{s}=0$ & $b_{f s}=0$ \\
\hline
\end{tabular}

Fig. 6. Numerical example 1. Material parameters chosen for the constitutive model in equation [21].

where $n_{\mathcal{Y}}$ denotes the total number of nodes within an element for the set $\mathcal{Y}$.

\section{Numerical examples}

A series of examples are included in this section in order to verify the robustness and applicability of the mixed formulations MFA- $T_{a}$ and MFA- $\gamma$ presented in Sections 5.2.1 and 5.2.2, respectively. One important aspect which is common to both formulations needs to be highlighted. Specifically, the reader must notice that the strain energy in (18) does not depend on the cofactor $\boldsymbol{H}$. Therefore, in this particular case, the weak form $\mathcal{W}_{\boldsymbol{H}}$ in (62) and its associated residual vector $\boldsymbol{R}_{\boldsymbol{H}}^{a}$ (72) are unnecessary, and so is the field $\boldsymbol{\Sigma}_{\boldsymbol{H}}$. Therefore, in the forthcoming numerical examples, the unknown fields for both MFA- $T_{a}$ and MFA- $\gamma$ formulations when considering the model in (18) will include $\{\boldsymbol{x}, \phi, p\},\{\boldsymbol{F}, \boldsymbol{H}, J, \boldsymbol{A}\}$ and $\left\{\boldsymbol{\Sigma}_{\boldsymbol{F}}, \Sigma_{J}, \boldsymbol{\Sigma}_{\boldsymbol{A}}\right\}$. On the contrary, for those examples where the strain energy depends on the cofactor (see Section 7.3.3), the field $\boldsymbol{\Sigma}_{\boldsymbol{H}}$ is included as part of the set of unknowns.

\subsection{Numerical example 1: patch test}

The objective of this example is:

- O1.I Assessment of the correctness of the computational implementation of the mixed formulations MFA- $T_{a}$ and MFA- $\gamma$ introduced in Sections 5.2.1 and 5.2.2 when using both Finite Elements ELEM1 and ELEM2 in Fig. 4.

In this example, we consider a portion of the myocardium defined by a cubic shaped domain of unit length. This domain is discretised using two $(3 \times 3 \times 3) \times 6$ tetrahedral discretisations characterised by: (a) structured mesh (refer to Fig. 5(a)) and (b) unstructured mesh (refer to Fig. 5(b)) where an arbitrary displacement of the interior nodes of the structured mesh is applied.

The constitutive model describing the passive response of the myocardium in this example is that in (18), and all the material parameters chosen can be found in Fig. 6 . In this example, the fibres $\left\{\boldsymbol{n}_{0}, \boldsymbol{s}_{0}, \boldsymbol{f}_{0}\right\}$ are chosen parallel to the axes $\{O X, O Y, O Z\}$, respectively. 


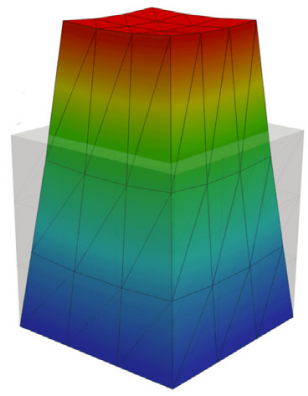

(a)

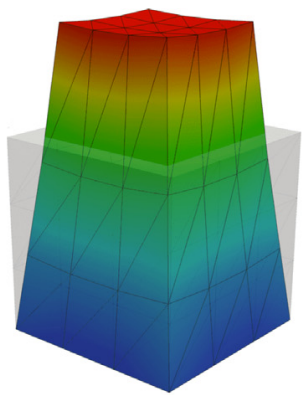

(e)

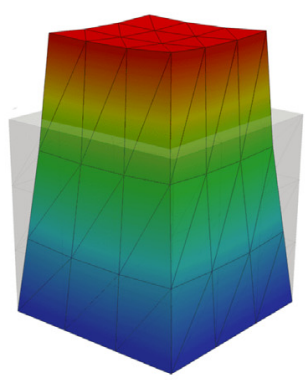

(b)

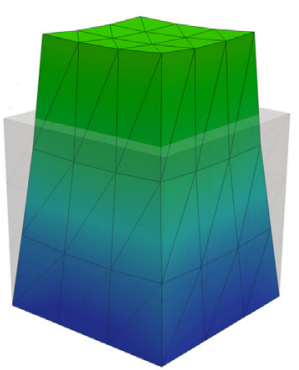

(f)

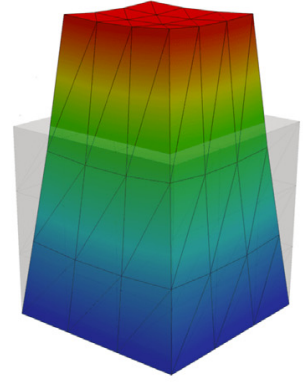

(c)

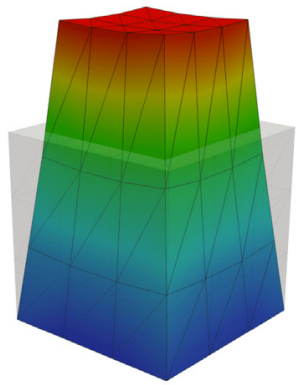

(g)

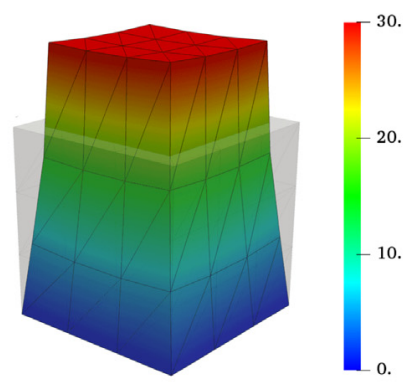

(d)

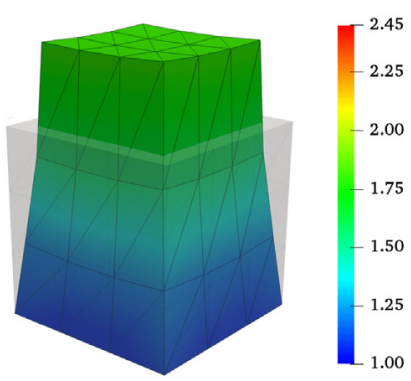

(h)

Fig. 7. Numerical example 1. Contour plot of $\phi$ on unstructured mesh for: (a) ELEM1-MFA- $T_{a}$; (b) ELEM1-MFA- $\gamma$; (c) ELEM2-MFA- $T_{a}$; (d) ELEM2-MFA- $\gamma$. Contour plot of $F_{z Z}$ on unstructured mesh for: (e) ELEM1-MFA- $T_{a}$; (f) ELEM1-MFA- $\gamma$; (g) ELEM2-MFA- $T_{a}$; (h) ELEM2MFA- $\gamma$.

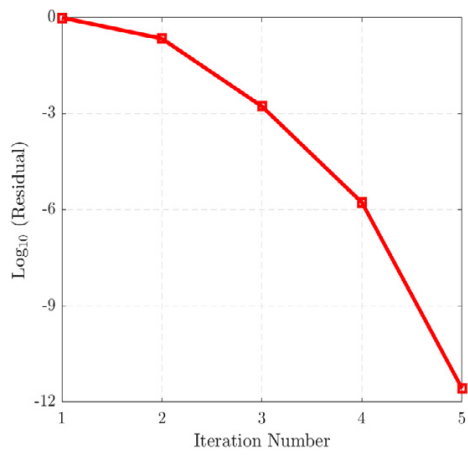

(a)

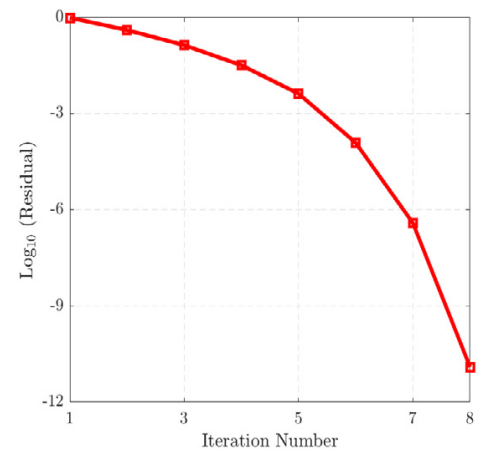

(b)

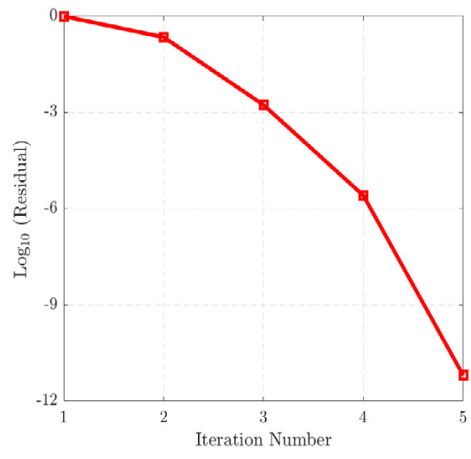

(c)

Fig. 8. Numerical example 1. Quadratic convergence of the Newton-Raphson algorithm for: (a) ELEM1-MFA- $\gamma$; (b) ELEM2-MFA- $\gamma$; (c) MFA$T_{a}$. All the results correspond to the unstructured mesh in Fig. $5_{b}$.

With regard to boundary conditions for the transmembrane potential $\phi$, this is prescribed as $\phi=0 \mathrm{mV}$ and $\phi=30 \mathrm{mV}$ on the faces perpendicular to the $O Z$ axis whilst homogeneous Neumann boundary conditions are applied elsewhere. Furthermore, homogeneous normal Dirichlet boundary conditions for the displacements are applied on the base perpendicular to the $O Z$ axis whilst homogeneous Neumann boundary conditions are applied elsewhere.

In order to focus strictly on above objective O1.I, the physics of the problem has been simplified. Specifically, the time derivative of the electric potential, i.e. $\dot{\phi}$ and the source term $f_{\phi}$ in (4) have been neglected. Furthermore, $a d$-hoc 
activation laws for both active stress and active strain approaches have been defined as

$$
T_{a}=\alpha \phi ; \quad \gamma_{f_{0}}=\beta \phi ; \quad \gamma_{s_{0}}=0 ; \quad \gamma_{n_{0}}=0,
$$

with $\alpha=-0.5$ and $\beta=0.05$.

As it can be seen from Fig. 7, a linear distribution for both the deformation gradient tensor $\boldsymbol{F}$ and the transmembrane potential $\phi$ are obtained along the $O Z$ axis, as expected. This distribution is identical for both structured and unstructured meshes and for both formulations MFA- $T_{a}$, MFA- $\gamma$, when using the Finite Elements ELEM1 and ELEM2 in Fig. 4. Therefore, both Finite Elements pass the patch test in both mixed formulations. For completeness, the quadratic convergence of the Newton-Raphson algorithm is shown in Fig. 8.

\subsection{Numerical example 2: convergence of new mixed formulations}

The objective of this example is:

- O2.I Demonstrate the $p$-order of accuracy of the mixed formulations MFA- $\gamma$ presented in Section 5.2.2 when using both Finite Elements ELEM1 and ELEM2.

The same cubic domain of unit length as in the previous example is considered. The constitutive model describing the passive response in this example is that in Eq. (18), with material parameters chosen as in Fig. 6. Furthermore, the directions of the fibres $\left\{\boldsymbol{f}_{0}, \boldsymbol{s}_{0}, \boldsymbol{n}_{0}\right\}$ are chosen parallel to the axes $\{O X, O Y, O Z\}$, respectively.

In order to study objective O2.I, the analysis of an ad hoc manufactured problem is carried out following a similar procedure as that described in reference [60]. For that, the following exact fields associated with the deformed configuration $\boldsymbol{x}$ and the transmembrane potential $\phi$ are chosen

$$
\boldsymbol{x}^{\text {exact }}=\boldsymbol{X}+\left[\begin{array}{c}
A X_{1}^{3} \\
B X_{2}^{3} \\
C X_{3}^{3}
\end{array}\right] ; \quad \phi^{\text {exact }}=D X_{1}^{4},
$$

with $\{A, B, C, D\}=\{0.01,0.01,0.01,100\}$. The exact deformation gradient tensor $\boldsymbol{F}^{\text {exact }}$ associated with $\boldsymbol{x}^{\text {exact }}$ and the material gradient of $\phi^{\text {exact }}$ can be computed as

$$
\boldsymbol{F}^{\text {exact }}=\left[\begin{array}{ccc}
1+3 A X_{1}^{2} & 0 & 0 \\
0 & 1+3 B X_{2}^{2} & 0 \\
0 & 0 & 1+3 C X_{3}^{2}
\end{array}\right] ; \quad \boldsymbol{A}^{\text {exact }}=\left[\begin{array}{c}
4 D X_{1}^{3} \\
0 \\
0
\end{array}\right],
$$

This enables to obtain the exact fields $\left\{\boldsymbol{H}^{\text {exact }}, J^{\text {exact }}, \boldsymbol{D}^{\text {exact }}\right\}$ using Eqs. (1) and (44) as

$$
\boldsymbol{H}^{\text {exact }}=\frac{1}{2} \boldsymbol{F}^{\text {exact }} \times \boldsymbol{F}^{\text {exact }} ; \quad J^{\text {exact }}=\frac{1}{3} \boldsymbol{H}^{\text {exact }}: \boldsymbol{F}^{\text {exact }} ; \quad D^{\text {exact }}=d_{\text {iso }} \frac{\left(\boldsymbol{H}^{\text {exact }}\right)^{T} \boldsymbol{H}^{\text {exact }}}{\left(J^{\text {exact }}\right)^{2}} .
$$

The following ad-hoc activation law for the active strain approach is considered

$$
\gamma_{f_{0}}^{\text {exact }}=\frac{\beta\left(\phi^{\text {exact }}\right)^{2}}{\left(\phi^{\text {exact }}\right)^{2}+1} ; \quad \gamma_{s_{0}}^{\text {exact }}=\gamma_{n_{0}}^{\text {exact }}=\frac{1}{\sqrt{\gamma_{f_{0}}^{\text {exact }}-1}},
$$

with $\beta=-0.1$. This enables us to compute the active component of the deformation tensor $\boldsymbol{F}^{A \text {,exact }}$ from (29) and its elastic counterpart $\boldsymbol{F}^{E \text {,exact }}$ from (28). With this, the first exact first Piola-Kirchhoff stress tensor $\boldsymbol{P}^{\text {exact }}$ can be obtained as in (36). In addition, using $d_{\text {iso }}=10^{-4}$ and $d_{\text {ani }}=0$ it is possible to compute $\boldsymbol{Q}^{\text {exact }}$ from (43)-(44). The computation of both $\left\{\boldsymbol{P}^{\text {exact }}, \boldsymbol{Q}^{\text {exact }}\right\}$, allows to obtain the associated volumetric force $\boldsymbol{b}_{0}$ in (3) and the source term $f_{\phi}$ in (4) as

$$
\boldsymbol{b}_{0}=\operatorname{DIV} \boldsymbol{P}^{\text {exact }} ; \quad f_{\phi}=-\operatorname{DIV} \boldsymbol{Q}^{\text {exact }},
$$

where the time dependent term $\dot{\phi}$ (refer to (4)) vanishes from the definition of $\phi^{\text {exact }}$ in (93). Dirichlet boundary conditions compatible with the exact fields $\left\{\boldsymbol{x}^{\text {exact }}, \phi^{\text {exact }}\right\}$ in (93) are applied on the boundary of the domain. Finally, 


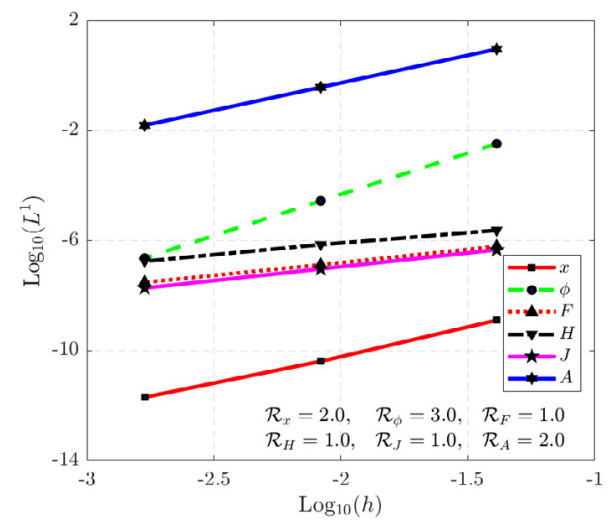

(a)

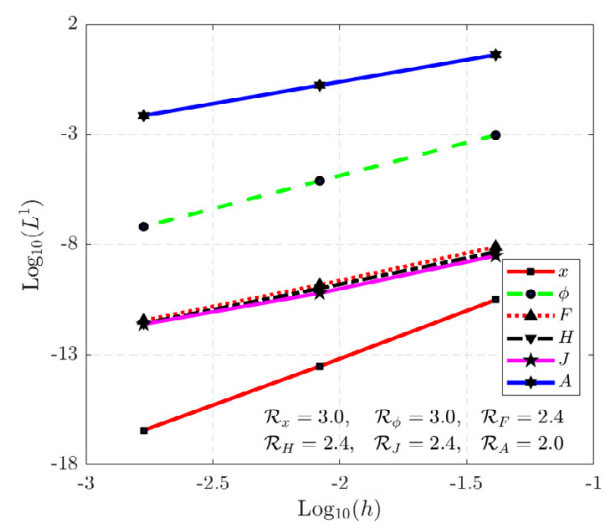

(c)

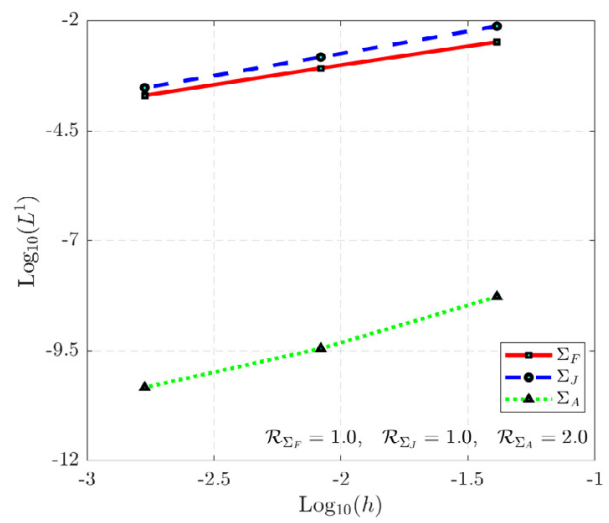

(b)

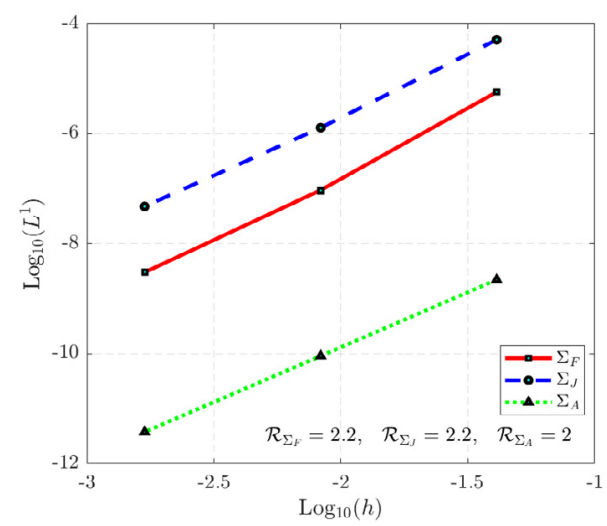

(d)

Fig. 9. Numerical example 2. $p$-order of accuracy for: $\{\boldsymbol{x}, \phi, \boldsymbol{F}, \boldsymbol{H}, J, \boldsymbol{A}\}$ for: (a) ELEM1-MFA- $\gamma$ and (c) ELEM2-MFA- $\gamma$. $p$-order of accuracy for: $\left\{\boldsymbol{\Sigma}_{\boldsymbol{F}}, \Sigma_{J}, \boldsymbol{\Sigma}_{\boldsymbol{A}}\right\}$ for: (b) ELEM1-MFA- $\gamma$ and (d) ELEM2-MFA- $\gamma$.

as it can be seen from the definition of $\boldsymbol{F}^{\text {exact }}$ in (94), the exact displacement field is not incompressible. Therefore, the pressure field $p$ will not be included as an unknown field for this specific example.

It can be seen from Fig. 9 that the convergence of the fields $\left\{\boldsymbol{x}, \boldsymbol{F}, \boldsymbol{H}, \boldsymbol{\Sigma}_{\boldsymbol{F}}, \boldsymbol{\Sigma}_{\boldsymbol{H}}\right\}$ is decreased by 1 due to the constant interpolation of the fields $\left\{J, \Sigma_{J}\right\}$. This has already been shown in Reference [60]. The remaining fields converge at the expected rate. On the contrary, for the Finite Element ELEM2, all the fields converge at the expected rate. Nonetheless, it has been shown (in the context of nonlinear elasticity, where no electrical coupling is present) $[60,77]$ that the equivalent to ELEM1 outperforms the classical $P_{2}$ (tetrahedral) element in bending dominated problems. Moreover, ELEM1 is computationally more effective than ELEM2 as the inclusion of bubble functions in the latter requires an increased order of quadrature with respect to ELEM1. Therefore, ELEM1 will be used for the remaining examples of this paper.

\subsection{Numerical example 3: benchmark problems}

The objective of this example is:

- O3.I Compare the accuracy of the new formulations presented in Sections 5.2.1 and 5.2.2 against available computer codes in the context of cardiac mechanics in a series of tailor-made benchmark examples reported in [78]. 


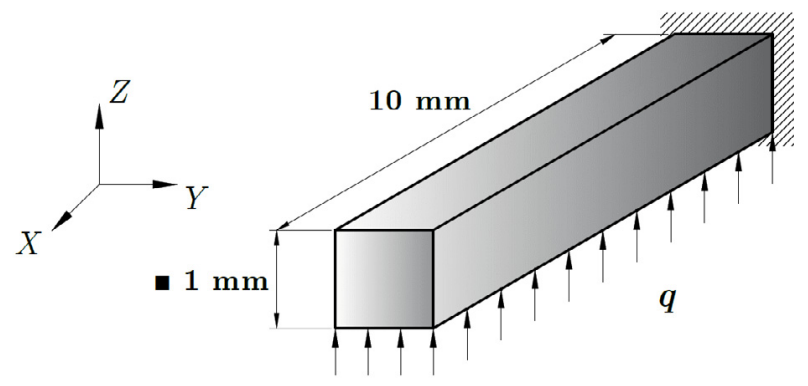

Fig. 10. Numerical example 3.1. Geometry and boundary conditions.
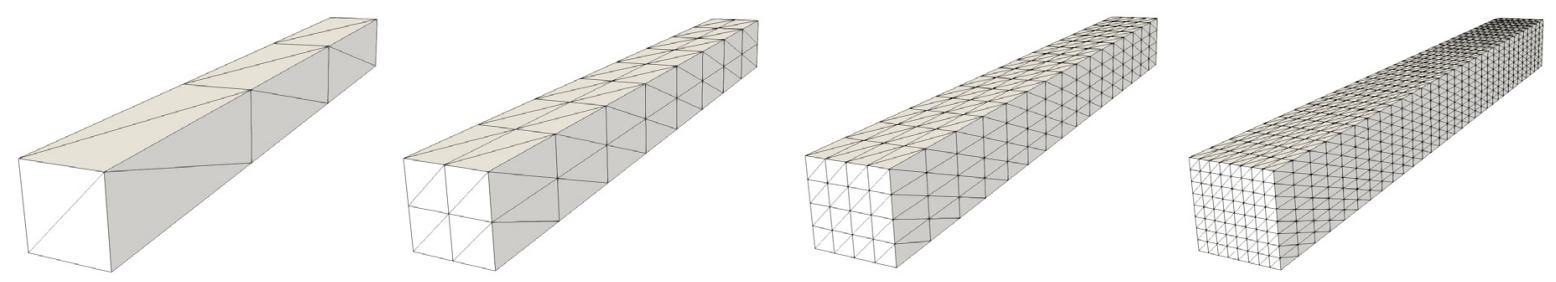

Fig. 11. Numerical example 3.1. Increasingly finer Finite Element discretisations for cantilever beam example. From left to right: $(1 \times 1 \times 3) \times 6$, $(2 \times 2 \times 8) \times 6,(4 \times 4 \times 16) \times 6$ and $(8 \times 8 \times 32) \times 6$ tetrahedral elements.

With the aim of aiding in the verification of current and future cardiac mechanics solvers, the authors in [78] proposed three benchmark problems for cardiac mechanics. In these, the constitutive model chosen for the passive response of the myocardium was that proposed by Guccione [19], defined as

$$
\Psi\left(\boldsymbol{E}^{\star}\right)=\frac{K}{2}\left(e^{Q\left(\boldsymbol{E}^{\star}\right)}-1\right),
$$

The exponent $Q\left(\boldsymbol{E}^{\star}\right)$ in (98) is defined in terms of the local Green-Lagrange strain tensor ${ }^{7}$ as

$$
Q\left(\boldsymbol{E}^{\star}\right)=c_{f}{E^{\star 2}}_{11}+c_{t}\left(E_{22}^{\star 2}+E_{33}^{\star 2}+E_{23}^{\star 2}+E_{32}^{\star 2}\right)+c_{f s}\left(E_{12}^{\star 2}+E_{21}^{\star 2}+E_{13}^{\star 2}+E_{31}^{\star 2}\right),
$$

where $\left\{K, c_{f}, c_{t}, c_{f s}\right\}$ represent material parameters. Alternatively, we have derived in Appendix B an invariant representation of $\boldsymbol{Q}\left(\boldsymbol{E}^{\star}\right)$ and an additional representation in terms of the (global) strain measures $\left\{\boldsymbol{F}_{\boldsymbol{x}}, \boldsymbol{H}_{\boldsymbol{x}}, J_{\boldsymbol{x}}\right\}$ as

$$
Q\left(\boldsymbol{E}^{\star}\right)=\widetilde{Q}\left(\boldsymbol{F}_{\boldsymbol{x}}, \boldsymbol{H}_{\boldsymbol{x}}, J_{\boldsymbol{x}}\right)=A \widetilde{Q}_{\mathrm{aniso}, 1}\left(\boldsymbol{F}_{\boldsymbol{x}}\right)+B \widetilde{Q}_{\text {aniso }, 2}\left(\boldsymbol{F}_{\boldsymbol{x}}, \boldsymbol{H}_{\boldsymbol{x}}\right)+C \widetilde{Q}_{\text {iso }}\left(\boldsymbol{F}_{\boldsymbol{x}}, \boldsymbol{H}_{\boldsymbol{x}}, \boldsymbol{J}_{\boldsymbol{x}}\right),
$$

with $\{A, B, C\}$ and $\left\{\widetilde{Q}_{\text {aniso, } 1}, \widetilde{Q}_{\text {aniso,2 }}, \widetilde{Q}_{\text {iso }}\right\}$ presented in Appendix B. Notice that above representation in (100) is more amenable for Finite Element implementation as it does not depend on the local axis of choice. Specifically, this representation permits the use of both mixed formulations presented in this paper, namely MFA- $T_{a}$ and MFA- $\gamma$ in Sections 5.2.1 and 5.2.2 in conjunction with Guccione's model, as these formulations are tailor-made for constitutive models expressed in terms of the strain measures $\left\{\boldsymbol{F}_{\boldsymbol{x}}, \boldsymbol{H}_{\boldsymbol{x}}, J_{\boldsymbol{x}}\right\}$.

\subsubsection{Numerical example 3.1: cantilever beam}

The first benchmark problem considers the beam with geometry and boundary conditions in Fig. 10. The beam is subjected to a follower load of $q=4 \mathrm{~Pa}$. The material parameters of Guccione's model in (98) are defined by $\left\{K, c_{f}, c_{t}, c_{f s}\right\}=\{2 \mathrm{kPa}, 8,2,4\}$ where the local axis $\left\{\boldsymbol{f}_{0}, \boldsymbol{s}_{0}, \boldsymbol{n}_{0}\right\}$ is chosen to be coincident with the global axis.

The four meshes in Fig. 11 will be used. Each mesh will be solved using the mixed formulation MFA- $T_{a}$ in Section 4.1.2 in conjunction with the Finite Element ELEM1 in Fig. 4. Notice that in this problem, electro-activation effects are neglected. Therefore, MFA- $T_{a}$ and MFA- $\gamma$ in Section 4.1.3 are identical in this specific scenario.

The results obtained with the ELEM1-MFA- $T_{a}$ formulation will be studied for two variables of interest: (a) the maximum Z-displacement for the four meshes in Fig. 11 will be compared against the results from authors in [78];

\footnotetext{
7 Let $\boldsymbol{R}$ be the rotation matrix from a local system of coordinates parallel to $\left\{\boldsymbol{f}_{0}, \boldsymbol{s}_{0}, \boldsymbol{n}_{0}\right\}$ to the global system of coordinates. $\boldsymbol{E}^{\star}$ can be related to its global counterpart $\boldsymbol{E}=\frac{1}{2}\left(\boldsymbol{F}_{x}^{T} \boldsymbol{F}_{\boldsymbol{x}}-\boldsymbol{I}\right)$ as $\boldsymbol{E}^{\star}=\boldsymbol{R}^{T} \boldsymbol{E} \boldsymbol{R}$.
} 


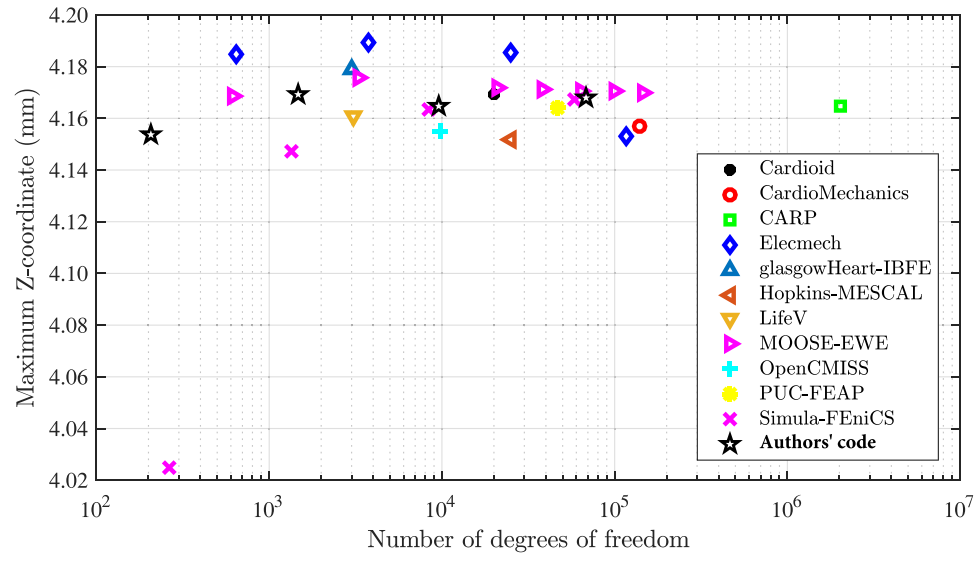

Fig. 12. Numerical example 3.1. Maximum $Z$-coordinate in the deformed configuration for the four discretisations in Fig. 11. Results obtained with ELEM1-MFA- $T_{a}$ formulation and by authors in Reference [78].
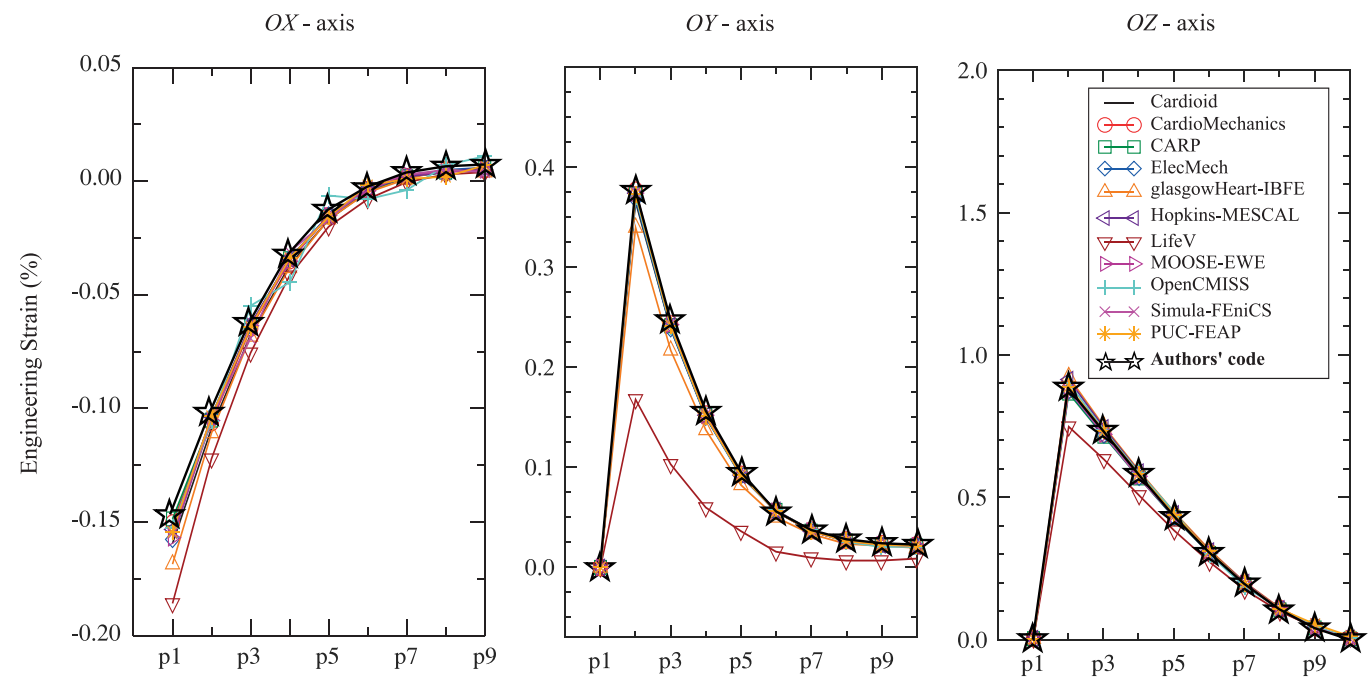

Fig. 13. Numerical example 3.1. Engineering strain $\varepsilon_{X}$ (left), $\varepsilon_{Y}$ (centre) and $\varepsilon_{Z}$ (right) for finest mesh in Fig. 11. $\left\{p_{1}, \ldots, p_{9}\right\}$ correspond to: $\left\{\overline{\boldsymbol{X}}_{X_{1}}, \ldots, \overline{\boldsymbol{X}}_{X_{9}}\right\}$ in left figure (refer to (102)); $\left\{\overline{\boldsymbol{X}}_{Y_{1}}, \ldots, \overline{\boldsymbol{Y}}_{X_{9}}\right\}$ in centre figure; $\left\{\overline{\boldsymbol{X}}_{Z_{1}}, \ldots, \overline{\boldsymbol{X}}_{Z_{9}}\right\}$ in right figure. Results obtained with ELEM1MFA- $T_{a}$ formulation and by authors in [78].

(b) the engineering strain measure along the three Cartesian directions for the finest mesh in Fig. 11 will be compared with other approaches. The engineering strain measures with respect to the axis $\{O X, O Y, O Z\}$ are defined at the material points $\left\{\overline{\boldsymbol{X}}_{X_{i}}, \overline{\boldsymbol{X}}_{Y_{i}}, \overline{\boldsymbol{X}}_{Z_{i}}\right\}$ as

$$
\varepsilon_{X}\left(\overline{\boldsymbol{X}}_{X_{i}}\right)=\frac{\left\|\hat{\boldsymbol{x}}_{i}-\overline{\boldsymbol{x}}_{X_{i}}\right\|}{\left\|\hat{\boldsymbol{X}}_{i}-\overline{\boldsymbol{X}}_{X_{i}}\right\|}-1 ; \quad \varepsilon_{Y}\left(\overline{\boldsymbol{X}}_{Y_{i}}\right)=\frac{\left\|\hat{\boldsymbol{x}}_{i}-\overline{\boldsymbol{x}}_{Y_{i}}\right\|}{\left\|\hat{\boldsymbol{X}}_{i}-\overline{\boldsymbol{X}}_{Y_{i}}\right\|}-1 ; \quad \varepsilon_{Z}\left(\overline{\boldsymbol{X}}_{Z_{i}}\right)=\frac{\left\|\hat{\boldsymbol{x}}_{i}-\overline{\boldsymbol{x}}_{Z_{i}}\right\|}{\left\|\hat{\boldsymbol{X}}_{i}-\overline{\boldsymbol{X}}_{Z_{i}}\right\|}-1,
$$

where the points $\left\{\hat{\boldsymbol{X}}_{i}, \overline{\boldsymbol{X}}_{X_{i}}, \overline{\boldsymbol{X}}_{Y_{i}}, \overline{\boldsymbol{X}}_{Z_{i}}\right\}$ in the reference configuration are defined as

$$
\begin{aligned}
& \hat{\boldsymbol{X}}_{i}=(i, 0.5,0.5) ; \quad \overline{\boldsymbol{X}}_{X_{i}}=(i+1,0.5,0.5) ; \quad i=\{0,1, \ldots, 9\} ; \\
& \overline{\boldsymbol{X}}_{Y_{i}}=(i, 0.9,0.5) ; \quad \overline{\boldsymbol{X}}_{Z_{i}}=(i, 0.5,0.9) ; \quad i=\{0,1, \ldots, 9\},
\end{aligned}
$$

and with $\left\{\hat{\boldsymbol{x}}_{i}, \overline{\boldsymbol{x}}_{X_{i}}, \overline{\boldsymbol{x}}_{Y_{i}}, \overline{\boldsymbol{x}}_{Z_{i}}\right\}$ their respective counterparts in the deformed configuration.

It can be seen from Fig. 12 that the maximum $Z$-coordinate obtained for the four discretisations is very similar. In fact, for the coarsest mesh, the results are considerably better than those obtained by the code Simula-FEniCS. In 

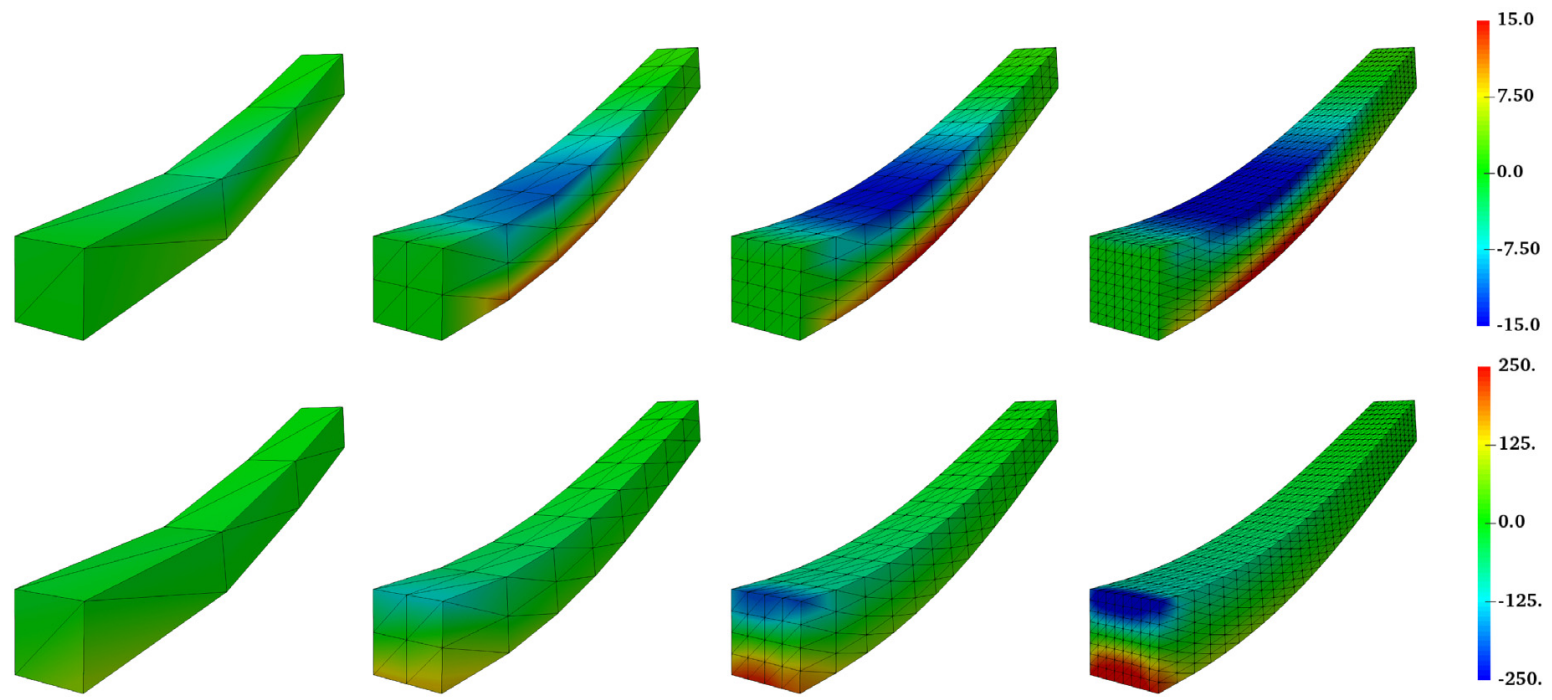

Fig. 14. Numerical example 3.1. Contour plot distribution of $P_{z X}$ and the Lagrange multiplier for incompressibility $p$ for the four discretisations considered and for the ELEM1-MFA- $T_{a}$ formulation (measured in Pa).

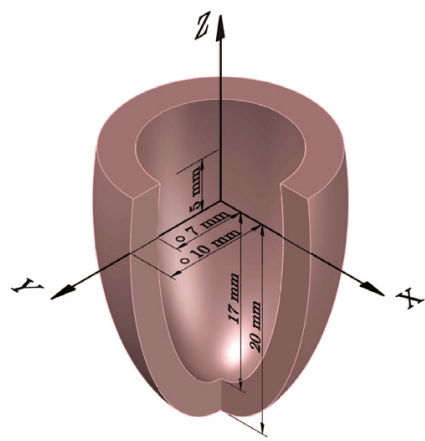

(a)

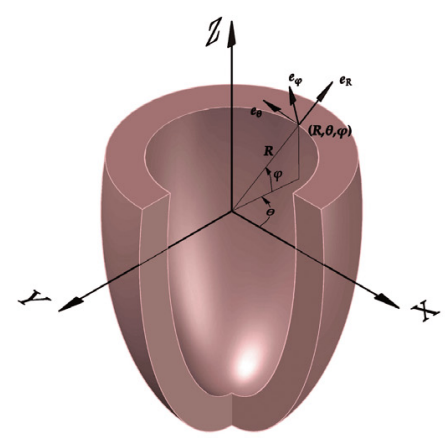

(b)

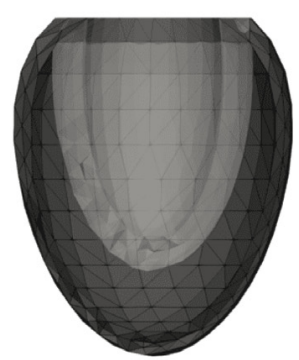

(c)

Fig. 15. Numerical example 3.2. (a) Geometry of the idealised ventricle. (b) Ellipsoidal coordinates of the idealised ventricle. (c) Initial configuration (grey) and final configuration (black) after inflation of the ventricle.

addition, Fig. 13 shows a good agreement of the results obtained by the ELEM1-MFA- $\gamma$ formulation with respect to those reported in [78] for the finest mesh in Fig. 11.

For the sake of completeness, the contour plot for the first Piola-Kirchhoff stress tensor $P_{z X}$ and the Lagrange multiplier $p$ enforcing the incompressibility constraint is shown in Fig. 14. A reasonable agreement for these variables is obtained for the four discretisations considered in this paper.

\subsubsection{Numerical example 3.2: inflation of a ventricle}

The second benchmark problem considers the idealised ventricle represented by an intersected set of ellipsoids with semiaxes $\{10,10,20\} \mathrm{mm}$ and $\{7,7,17\} \mathrm{mm}$ centred in the origin and truncated by the plane $Z=5 \mathrm{~mm}$ (see Fig. $15_{a}$ ). The material parameters of Guccione's model (98) are $\left\{K, c_{f}, c_{s}, c_{f s}\right\}=\{10 \mathrm{kPa}, 1,1,1\}$. Homogeneous Dirichlet boundary conditions are applied on the plane $Z=5 \mathrm{~mm}$ for all directions and a pressure (follower load) of $10 \mathrm{kPa}$ is applied on the inner face (endocardium), leading to the inflation of the ventricle (see Fig. $15_{c}$ ).

The engineering strain is defined now with respect to the ellipsoidal coordinates $\{\varphi, \theta, R\}$ of the ventricle (refer to Fig. 15). The engineering strains with respect to the axis $\{O \varphi, O \theta, O R\}$ at material points $\left\{\overline{\boldsymbol{X}}_{\varphi_{i}}, \overline{\boldsymbol{X}}_{\theta_{i}}, \overline{\boldsymbol{X}}_{R_{i}}\right\}$ are 
Table 1

Numerical example 3.2. Engineering strain $\varepsilon_{\theta}$ (left), $\varepsilon_{\phi}$ (centre) and $\varepsilon_{R}$ (right). $\left\{p_{1}, \ldots, p_{9}\right\}$ corresponds to: $\left\{\overline{\boldsymbol{X}}_{\theta_{1}}, \ldots, \overline{\boldsymbol{X}}_{\theta_{9}}\right\}$ in left figure (refer to [78]); $\left\{\overline{\boldsymbol{X}}_{\varphi_{1}}, \ldots, \overline{\boldsymbol{Y}}_{\varphi_{9}}\right\}$ in centre figure; $\left\{\overline{\boldsymbol{X}}_{R_{1}}, \ldots, \overline{\boldsymbol{X}}_{R_{9}}\right\}$ in right figure. Results obtained with ELEM1-MFA- $T_{a}$ formulation and by authors in [78].

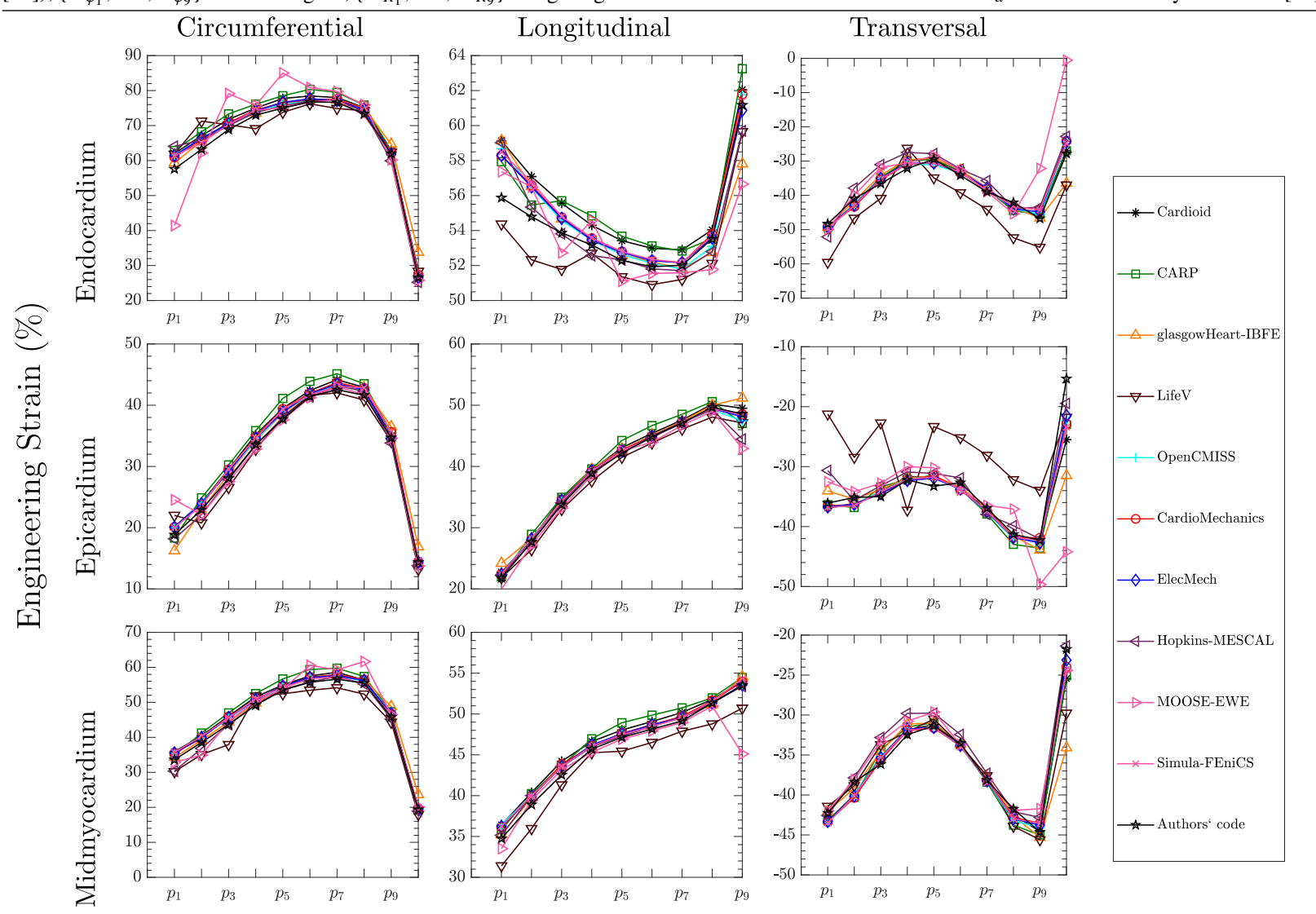

defined in a similar fashion to Eq. (101) as

$$
\varepsilon_{\varphi}\left(\overline{\boldsymbol{X}}_{\varphi_{i}}\right)=\frac{\left\|\hat{\boldsymbol{x}}_{i}-\overline{\boldsymbol{x}}_{\varphi_{i}}\right\|}{\left\|\hat{\boldsymbol{X}}_{i}-\overline{\boldsymbol{X}}_{\varphi_{i}}\right\|}-1 ; \quad \varepsilon_{\theta}\left(\overline{\boldsymbol{X}}_{\theta_{i}}\right)=\frac{\left\|\hat{\boldsymbol{x}}_{i}-\overline{\boldsymbol{x}}_{\theta_{i}}\right\|}{\left\|\hat{\boldsymbol{X}}_{i}-\overline{\boldsymbol{X}}_{\theta_{i}}\right\|}-1 ; \quad \varepsilon_{R}\left(\overline{\boldsymbol{X}}_{R_{i}}\right)=\frac{\left\|\hat{\boldsymbol{x}}_{i}-\overline{\boldsymbol{x}}_{R_{i}}\right\|}{\left\|\hat{\boldsymbol{X}}_{i}-\overline{\boldsymbol{X}}_{R_{i}}\right\|}-1,
$$

where the points $\left\{\hat{\boldsymbol{X}}_{i}, \overline{\boldsymbol{X}}_{\varphi_{i}}, \overline{\boldsymbol{X}}_{\theta_{i}}, \overline{\boldsymbol{X}}_{R_{i}}\right\}$ can be found in Reference [78]. Table 1 shows a good agreement of the results obtained by the ELEM1-MFA- $T_{a}$ formulation with respect to those reported in [78] for the three engineering strains $\left\{\varepsilon_{\varphi}, \varepsilon_{\theta}, \varepsilon_{R}\right\}$ for all the points described in Reference [78] located at the endocardium, midmyocardium and epicardium. Finally, Fig. 16 displays the contour plot of $\left\{F_{x Y}, H_{z Y}, P_{z X}, p, \Sigma_{F_{z Y}}, \Sigma_{J}\right\}$ by means of the ELEM1MFA- $T_{a}$ formulation.

\subsubsection{Numerical example 3.3: inflation and active contraction of a ventricle}

The geometry of the third example can be seen in Fig. $17_{a}$. The Dirichlet boundary conditions are identical to those in Section 7.3.2. In this case, there is a combined effect of a follower load of value $15 \mathrm{kPa}$ and a contraction along a set of fibres $\boldsymbol{f}_{0}$ whose parametrisation can be found in [78]. This contraction is imposed by considering a value of $60 \mathrm{kPa}$ for the cardiomyocite stress $T_{a}$ in the active stress approach. The proposed mathematical description of the fibres $\boldsymbol{f}_{0}$ is singular in the apex of the ventricle, leading to stress concentration in this region.

The material parameters of Guccione's constitutive law (98) are chosen as $\left\{K, c_{f}, c_{s}, c_{f s}\right\}=\{10 \mathrm{kPa}, 8,2,4\}$. Table 2 shows a good agreement of the results obtained by the ELEM1-MFA- $T_{a}$ formulation with respect 


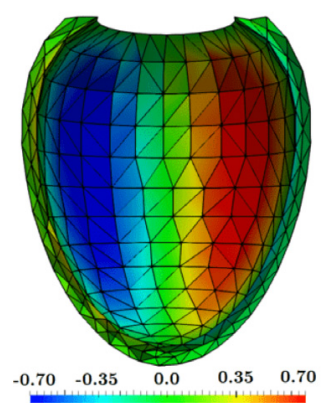

(a)

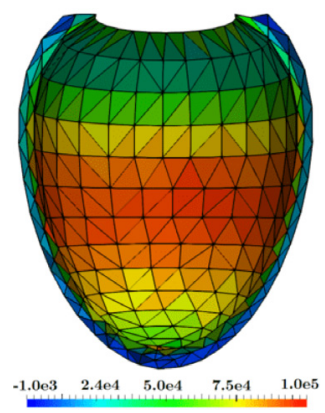

(d)

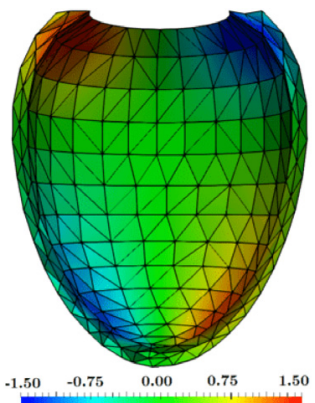

(b)

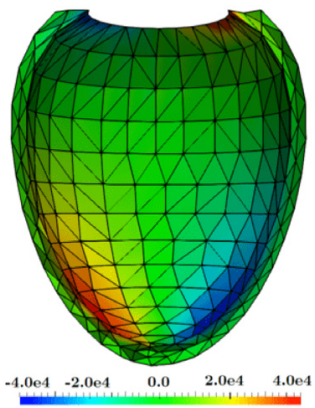

(e)

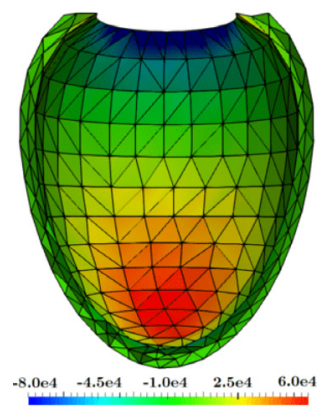

(c)

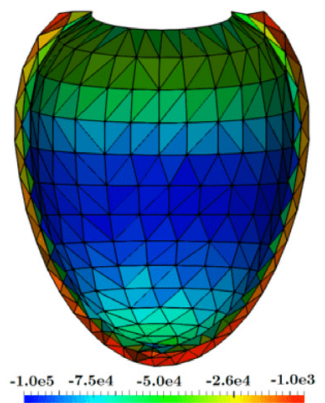

(f)

Fig. 16. Numerical example 3.2. Contour plot distribution of: (a) $F_{X Y}$, (b) $H_{z Y}$, (c) $P_{z X}$ (Pa), (d) $p$ (Pa), (e) $\Sigma_{F_{Z Y}}$ (Pa) and (f) $\Sigma_{J}$ (Pa). Results obtained with ELEM1-MFA- $T_{a}$ formulation.

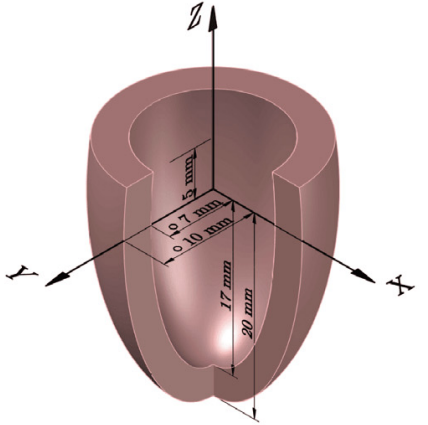

(a)

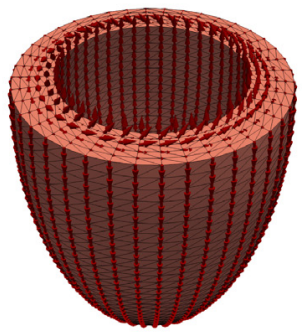

(b)

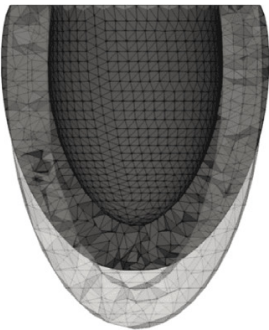

(c)

Fig. 17. Numerical example 3.3. (a) Geometry of the idealised ventricle. (b) Orientation of $\boldsymbol{f}_{0}$ in Guccione's model (B.1). (c) Undeformed (transparent) and deformed (grey) configurations after activation of the ventricle.

to those reported in [78] for the three engineering strains $\left\{\varepsilon_{\varphi}, \varepsilon_{\theta}, \varepsilon_{R}\right\}$ for all the points described in Reference [78] located at the endocardium, midmyocardium and epicardium. Finally, Fig. 18 displays the contour plot of $\left\{F_{z Y}, H_{z Y}, P_{z X}, p, \Sigma_{F_{z Y}}, \Sigma_{J}\right\}$ by means of the ELEM1-MFA- $T_{a}$ formulation.

\subsection{Numerical example 4: coupled electro-mechanical simulation of a pair of idealised ventricles}

The objectives of this example are: 
Table 2

Numerical example 3.3. Engineering strain $\varepsilon_{\theta}$ (left), $\varepsilon_{\phi}$ (centre) and $\varepsilon_{R}$ (right). $\left\{p_{1}, \ldots, p_{9}\right\}$ corresponds to: $\left\{\overline{\boldsymbol{X}}_{\theta_{1}}, \ldots, \overline{\boldsymbol{X}}_{\theta_{9}}\right\}$ in left figure (refer to [78]); $\left\{\overline{\boldsymbol{X}}_{\varphi_{1}}, \ldots, \overline{\boldsymbol{Y}}_{\varphi_{9}}\right\}$ in centre figure; $\left\{\overline{\boldsymbol{X}}_{R_{1}}, \ldots, \overline{\boldsymbol{X}}_{R_{9}}\right\}$ in right figure. Results obtained with ELEM1-MFA- $T_{a}$ formulation and by authors in [78].

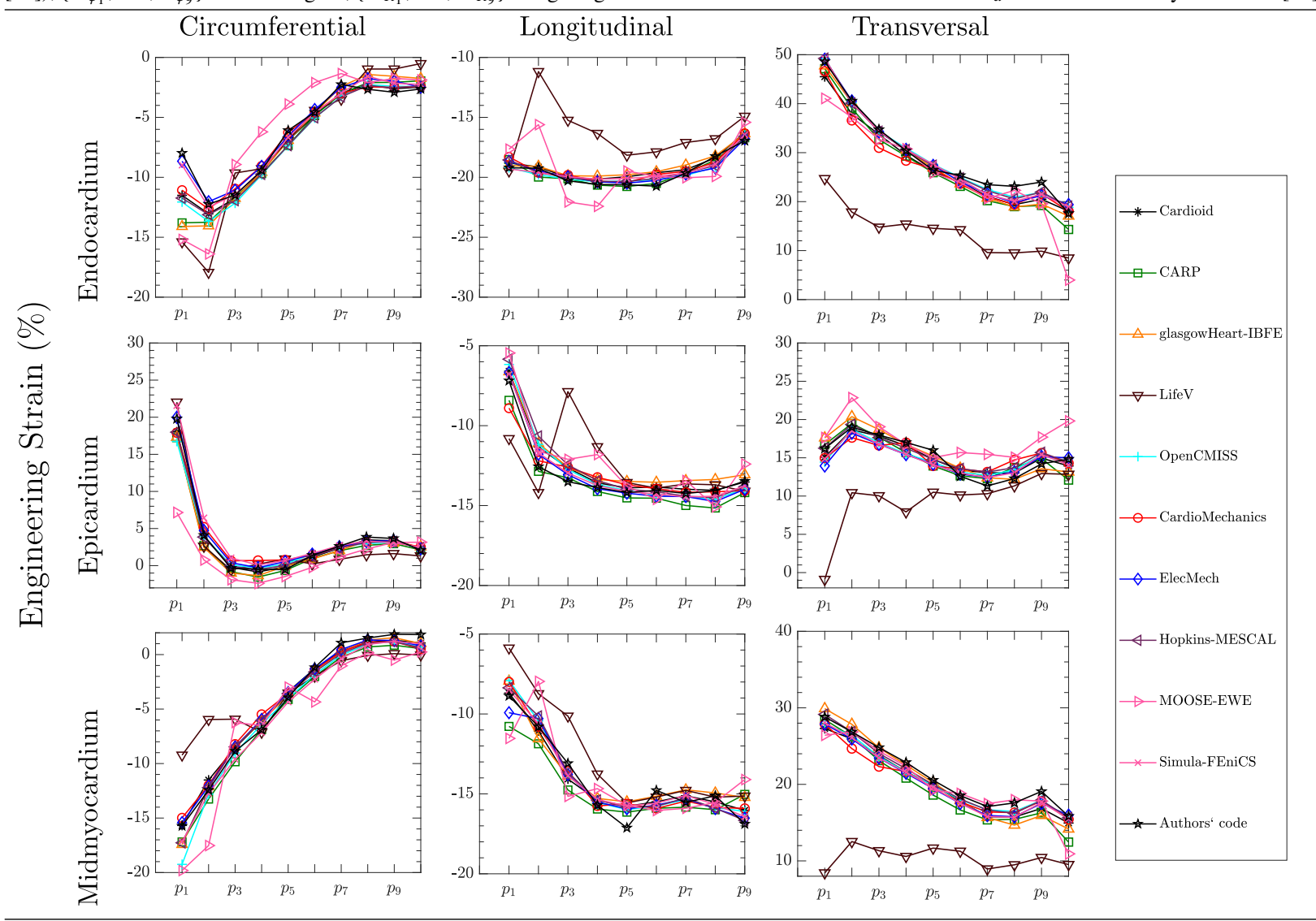

- O4.I Demonstrate the applicability of formulations MFA- $T_{a}$ in Section 5.2.1 and MFA- $\gamma$ in Section 5.2.2 for the simulation of the cardiac cycle under different: (a) coupling type approaches (active stress/active strain); (b) activation laws (see Section 4.3) and (c) ionic models (see Section 4.2).

- O4.II Demonstrate the shortcomings of conventional Finite Element approaches for the modelling of cardiac mechanics.

- O4.III Study the susceptibility for loss of ellipticity of the constitutive model proposed by Holzapfel-Ogden in [21] (refer to (18)) for both active strain and active stress approaches during the cardiac cycle.

The main features of this example are:

Geometry: we consider in this example an idealised geometry of two ventricles defined by four ellipsoids. The two outermost ellipsoids are centred in the origin, i.e $\{X, Y, Z\}=\{0,0,0\}$ and the length of their semi-axes is $\{50,50,70\}$ $\mathrm{mm}$ and $\{45,45,65\} \mathrm{mm}$. The two innermost ellipsoids are centred at $\{X, Y, Z\}=\{0,-10,0\}$ and the length of their semi-axes is $\{40,40,63\} \mathrm{mm}$ and $\{35,35,60\} \mathrm{mm}$. The four ellipsoids are truncated by the plane $Z=0$.

Fibres direction: we consider the surfaces described by the four ellipsoids of the ventricle. For each point of these surfaces, the unitary vector $f_{0}$ will be considered to be tangential to them. Specifically, at each point of them, $\boldsymbol{f}_{0}$ forms an angle of +60 or -60 degrees with respect to its local circumferential axis. The angle is +60 in the first (outermost ellipsoid) and third ellipsoids, whereas the angle is -60 in the remaining ellipsoids (refer to Fig. $19_{b}$ ). A Poisson problem is solved in order to obtain the orientation of $\boldsymbol{f}_{0}$ in the interior of the ventricles [10] (at Gauss point level). 


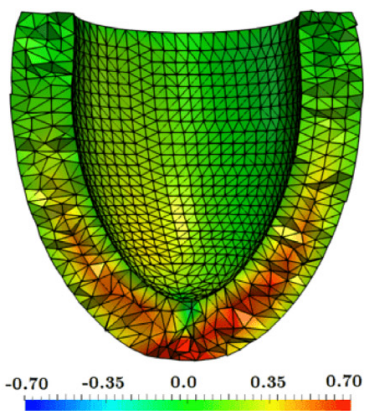

(a)

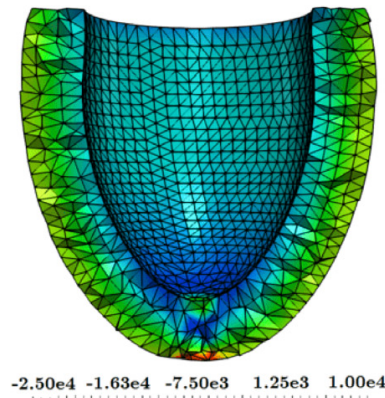

(d)

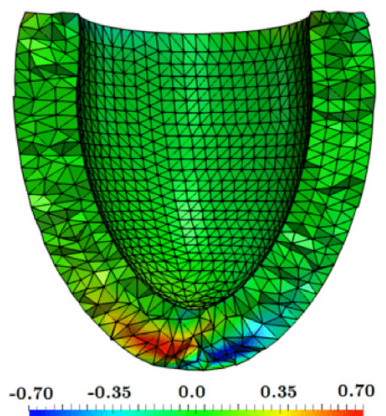

(b)

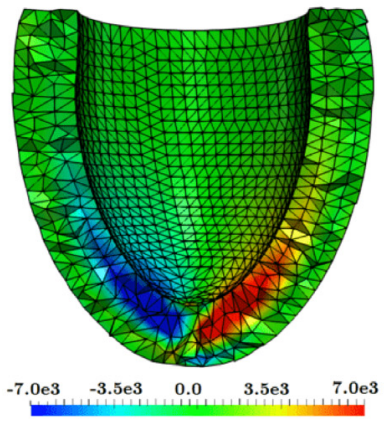

(e)

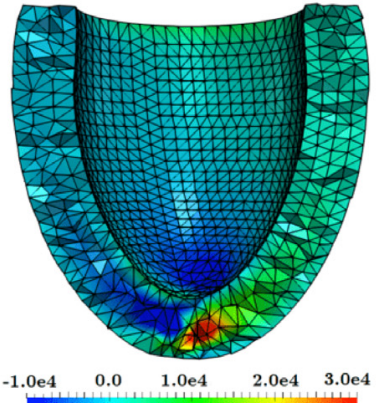

(c)

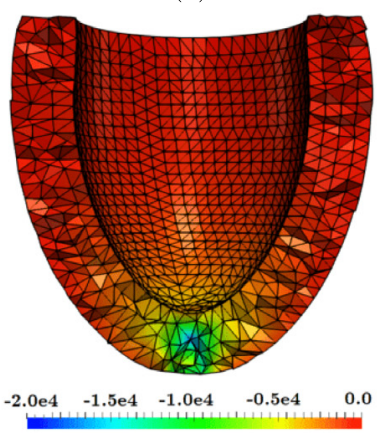

(f)

Fig. 18. Numerical example 3.3. Contour plot distribution of: (a) $F_{x Y}$, (b) $H_{z Y}$, (c) $P_{z X}(\mathrm{~Pa})$, (d) $p(\mathrm{~Pa})$, (e) $\Sigma_{F_{z Y}}(\mathrm{~Pa})$ and (f) $\Sigma_{J}(\mathrm{~Pa})$. Results obtained with the ELEM1-MFA- $T_{a}$ formulation.

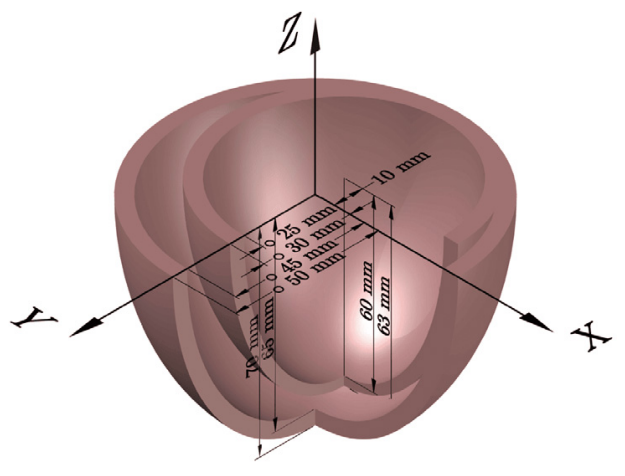

(a)

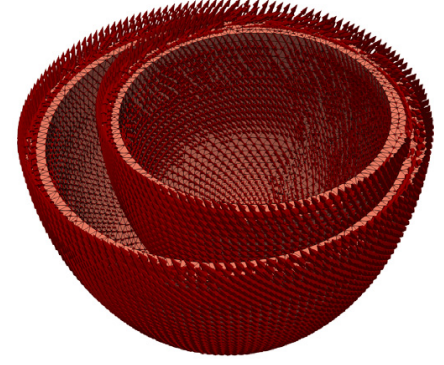

(b)

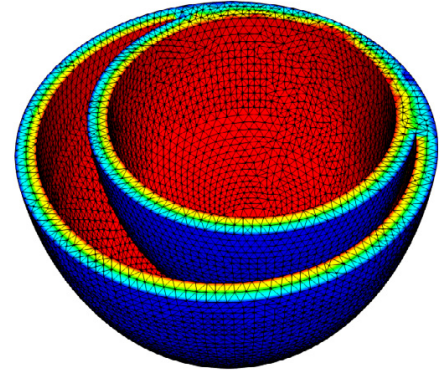

(c)

Fig. 19. Numerical example 4. (a) Geometry of the two ventricles. (b) Representation of the fibre $\boldsymbol{f}_{0}$. (c) Contour plot of $\eta$ for the interpolation of $f_{0}$. Blue and red regions correspond to $\eta=1$ and $\eta=0$, respectively. (For interpretation of the references to colour in this figure legend, the reader is referred to the web version of this article.)

This is done by introducing the intermediate field $\eta$, satisfying $\nabla_{0} \cdot\left(\nabla_{0} \eta\right)=0$ in $\Omega_{0}$, where $\eta=1$ in the ellipsoidal surfaces associated with a +60 orientation of $\boldsymbol{f}_{0}$ and $\eta=0$ in the remaining ellipsoidal surfaces (refer to Fig. $19 c$ ). In addition, this intermediate field helps to compute the normal unit vector $s_{0}$ as $\nabla_{0} \eta /\left\|\nabla_{0} \eta\right\|$. With all this information, 


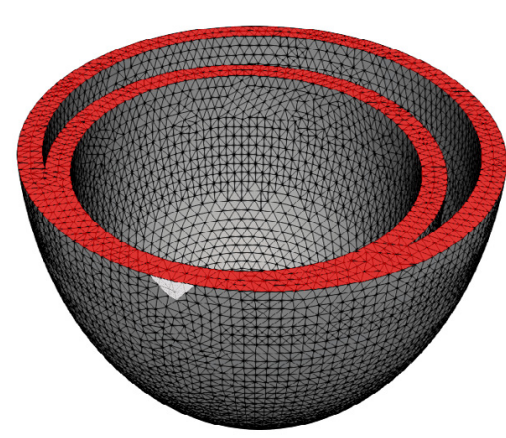

(a)

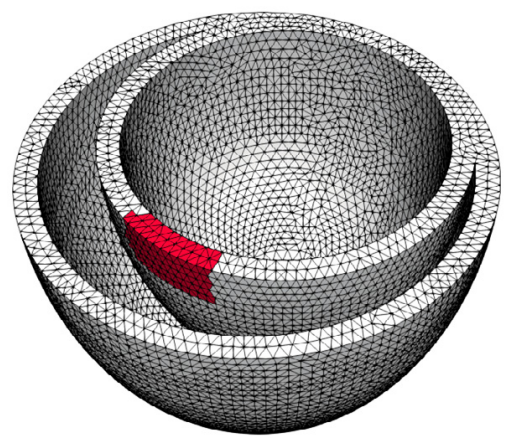

(b)

Fig. 20. Numerical example 4. (a) Regions for the application of homogeneous Dirichlet boundary conditions for displacements. In red: restriction displacement in $O Z$ direction; in white: restriction in all directions. (b) Region where the initial electrical stimulation occurs. (For interpretation of the references to colour in this figure legend, the reader is referred to the web version of this article.)

\begin{tabular}{|c|c|c|c|c|c|}
\hline \multicolumn{1}{|c|}{ Summary of details for the four simulation } \\
\hline Simulation name & Coupling type & Activation Law & Ionic Model & Passive response & Mixed Formulation \\
\hline Simulation-I & Active Stress & Nash \& Panfilov & Bueno-Orovio & Holzapfel-Ogden & ELEM1-MFA- $T_{a}$ \\
Simulation-II & Active Stress & Wong \& Kuhl & Ten Tusscher & Holzapfel-Ogden & ELEM1-MFA- $T_{a}$ \\
Simulation-III & Active Strain & Rossi et al. & Bueno-Orovio & Holzapfel-Ogden & ELEM1-MFA- $\gamma$ \\
Simulation-IV & Active Strain & Rossi et al. & Ten Tusscher & Holzapfel-Ogden & ELEM1-MFA- $\gamma$ \\
\hline
\end{tabular}

Fig. 21. Numerical example 4.1. Description of the four simulations considered.

the direction $\boldsymbol{f}_{0}$ is then calculated by means of Rodrigues' rotation formula as explained in Reference [7]. Finally, the remaining direction $\boldsymbol{n}_{0}$ is computed as $\boldsymbol{n}_{0}=\boldsymbol{f}_{0} \times \boldsymbol{s}_{0}$.

Boundary conditions: homogeneous Dirichlet boundary conditions are applied on the coloured regions in Fig. $20_{a}$. Homogeneous Neumann boundary conditions are applied elsewhere.

Initial conditions: the cardiac action potential is triggered by means of an arbitrary stimulation intensity $I_{\text {stim }}$ applied on the coloured region in Fig. $20_{b}$.

Time integration: an adaptive time step strategy is used for the time integration of Eq. (4). A small time step $\Delta t$ is required in order to correctly capture the rapid initial depolarisation and can then be gradually increased without compromising the accuracy of the simulation. The time step $\Delta t$ at a given time $t$ is automatically chosen by measuring the rate of change in absolute value of the internal variables in the ionic models (see Section 4.2). The minimum and maximum values for $\Delta t$ are $0.3 \mathrm{~ms}$ and $50 \mathrm{~ms}$, respectively. Furthermore, the time integration of Eq. (4), the evolution equations for the activation laws for $T_{a}$ (49) and $\gamma_{f_{0}}$ (52) and the ODE for the internal variables $\boldsymbol{q}$ in (45) is carried out by means of the (implicit) Backward-Euler scheme.

Material parameters: Fig. 22 contains the values for the relevant material parameters for: (a) Holzapfel-Ogden model [21] in (18); (b) activation model proposed by Nash and Panfilov [51]; (c) activation model proposed by 


\begin{tabular}{|c|c|c|c|}
\hline \multicolumn{4}{|c|}{ Parameters for the Holzapfel-Ogden constitutive law } \\
\hline$a=0.496 \mathrm{kPa}$ & $a_{f}=15.196 \mathrm{kPa}$ & $a_{s}=3.283 \mathrm{kPa}$ & $a_{f s}=0.662 \mathrm{kPa}$ \\
\hline$b=7.209$ & $b_{f}=20.417$ & $b_{s}=11.176$ & $b_{f s}=9.466$ \\
\hline
\end{tabular}

\begin{tabular}{|c|c|c|c|c|}
\hline Parameters for the activation model proposed by Nash and Panfilov \\
\hline$k_{T_{a}}=12.5 \mathrm{kPa} / \mu \mathrm{M}$ & - & - & - & - \\
\hline
\end{tabular}

\begin{tabular}{|c|c|c|c|c|c|}
\hline \multicolumn{6}{|c|}{ Parameters for the activation model proposed by Wong $e t$ al. } \\
\hline$\eta=12.5 \mathrm{kPa} / \mu \mathrm{M}$ & $c_{\mathrm{Ca}}^{\text {crit }}=0.8 \mu \mathrm{M}$ & $c_{\mathrm{Ca}, 0}=0.08 \mu \mathrm{M}$ & $\varepsilon_{0}=0.1 \mathrm{~ms}^{-1}$ & $\varepsilon_{\infty}=1 \mathrm{~ms}^{-1}$ & $\xi=4 \mu \mathrm{M}^{-1}$ \\
\hline
\end{tabular}

\begin{tabular}{|c|c|c|}
\hline \multicolumn{3}{|l|}{ Parameters for the activation law proposed by Rossi $\boldsymbol{e t}$ al. } \\
\hline$d_{1}=2570.395355352195$ & $e_{2}=302.216784558222$ & $d_{0}=-4333.618335582119$ \\
\hline$e_{1}=-2051.827278991976$ & $d_{3}=104.943770305116$ & $\hat{\mu}_{A}=5000 \mathrm{~ms} \mu \mathrm{M}^{-2}$ \\
\hline$d_{2}=1329.536116891330$ & $e_{3}=218.375174229422$ & $l_{0}=1.95 \mathrm{~mm} \quad \alpha=-4 \mu \mathrm{M}^{-2}$ \\
\hline
\end{tabular}

\begin{tabular}{|l|l|l|l|l|l|c|}
\hline \multicolumn{2}{|c|}{ Parameters for the Bueno-Orovio ionic model } \\
\hline$u_{o}=0.0$ & $\theta_{v}=0.3$ & $\tau_{o 1}=400$ & $\tau_{v 1}^{-}=60$ & $w_{\infty}^{*}=0.94$ & $\tau_{s 1}=2.7342$ & $k_{w}^{-}=65$ \\
\hline$u_{u}=1.55$ & $\theta_{w}=0.13$ & $\tau_{o 2}=6$ & $\tau_{v 2}^{-}=1150$ & $\tau_{w 1}^{-}=60$ & $\tau_{s 2}=16$ & $k_{s}=2.0994$ \\
\hline$u_{s}=0.9087$ & $\theta_{o}=0.006$ & $\tau_{s o 1}=30.0181$ & $\tau_{w}^{+}=200$ & $\tau_{w 2}^{-}=15$ & $\tau_{s i}=1.8875$ & $k_{s o}=2.0458$ \\
\hline$u_{w}^{-}=0.03$ & $\theta_{v}^{-}=0.006$ & $\tau_{s o 2}=0.9957$ & $\tau_{v}^{+}=1.4506$ & $\tau_{f i}=0.11$ & $\tau_{w}^{\infty}=0.07$ & $I_{\text {stim }}=0.9$ \\
\hline
\end{tabular}

\begin{tabular}{|c|c|c|c|}
\hline \multicolumn{5}{|c|}{ Parameters for the Ten Tusscher ionic model } \\
\hline$\alpha=2.5$ & $G_{\mathrm{Na}}=14.838 \mathrm{nS} / \mathrm{pF}$ & $K_{\mathrm{Buf}_{\mathrm{sr}}}=0.3 \mathrm{mM}$ & $V_{\mathrm{C}}=0.016404 \mathrm{~cm}^{3}$ \\
\hline$\gamma=0.35$ & $G_{\mathrm{K} 1}=5.405 \mathrm{nS} / \mathrm{pF}$ & $K_{\mathrm{up}}=0.00025 \mathrm{mM}$ & $V_{\mathrm{SR}}=0.001094 \mathrm{~cm}^{3}$ \\
\hline$T=310 \mathrm{~K}$ & $G_{\mathrm{to}}=0.245 \mathrm{nS} / \mathrm{pF}$ & $K_{\mathrm{mNai}}=87.5 \mathrm{mM}$ & $V_{\text {leak }}=0.00008 \mathrm{~ms}^{-1}$ \\
\hline$k_{\mathrm{sat}}=0.1$ & $G_{\mathrm{Kr}}=0.0096 \mathrm{nS} / \mathrm{pF}$ & $K_{\mathrm{pCa}}=0.0005 \mathrm{mM}$ & $a_{\text {rel }}=0.016464 \mathrm{mM} / \mathrm{ms}$ \\
\hline$p_{\mathrm{KNa}}=0.03$ & $G_{\mathrm{Ks}}=0.245 \mathrm{nS} / \mathrm{pF}$ & $K_{\mathrm{mK}}=1 \mathrm{mM}$ & $b_{\text {rel }}=0.25 \mathrm{mM}$ \\
\hline $\mathrm{Ca}_{\mathrm{O}}=2 \mathrm{mM}$ & $G_{\mathrm{pk}}=0.0146 \mathrm{nS} / \mathrm{pF}$ & $K_{\mathrm{mNa}}=40 \mathrm{mM}$ & $c_{\text {rel }}=0.008232 \mathrm{mM} / \mathrm{ms}$ \\
\hline$K_{\mathrm{O}}=5.4 \mathrm{mM}$ & $G_{\mathrm{pCa}}=0.825 \mathrm{nS} / \mathrm{pF}$ & $K_{\mathrm{mCa}}=1.38 \mathrm{mM}$ & $V_{\text {max,up }}=0.000425 \mathrm{mM} / \mathrm{ms}$ \\
\hline $\mathrm{Na}_{\mathrm{O}}=140 \mathrm{mM}$ & $G_{\mathrm{bCa}}=0.000592 \mathrm{nS} / \mathrm{pF}$ & $K_{\mathrm{Buf}}=0.001 \mathrm{mM}$ & $R=8.3143 \mathrm{~mJ} \mathrm{~K} / \mathrm{mM}$ \\
\hline $\mathrm{Buf}_{\mathrm{c}}=0.15 \mathrm{mM}$ & $G_{\mathrm{bNa}}=0.00029 \mathrm{nS} / \mathrm{pF}$ & $P_{\mathrm{NaK}}=1.362 \mathrm{pA} / \mathrm{pF}$ & $F=96.4867 \mathrm{C} / \mathrm{mM}$ \\
\hline $\mathrm{Buf}_{\mathrm{sr}}=10 \mathrm{mM}$ & $G_{\mathrm{CaL}}=0.175 \mathrm{~mm} / \mu \mathrm{F} / \mathrm{s}$ & $k_{\mathrm{NaCa}}=1000 \mathrm{pA} / \mathrm{pF}$ & $C_{\mathrm{m}}=0.000185 \mu \mathrm{F} / \mathrm{cm}^{2}$ \\
\hline
\end{tabular}

\section{Additional parameters}

$$
\begin{array}{l|l}
d_{\text {iso }}=8 \cdot 10^{-4} \mathrm{~m}^{2} / \mathrm{s} & d_{\text {ani }}=12 \cdot 10^{-4} \mathrm{~m}^{2} / \mathrm{s}
\end{array}
$$

Fig. 22. Numerical example 4. Parameters for: (a) Holzapfel-Ogden model [21] for passive response of the myocardium. Values provided in Göktepe et al. [38]; (b) Activation model proposed by Nash and Panfilov [51] (activation type: active stress; ionic model: Bueno-Orovio). (c) Activation model proposed by Wong et al. [52] (activation type: active stress; ionic model: Ten Tusscher). (d) Activation model proposed by Rossi et al. [7] (activation type: active strain; ionic model: both Bueno-Orovio and Ten Tusscher). (e) Ionic model proposed by Bueno-Orovio et al. [49]; (f) Ionic model proposed by Ten Tusscher et al. [48]. (g) Electrical conductivities in (63).

Wong et al. [52]. (d) activation model proposed by Rossi et al. [7]; (e) ionic model proposed by Bueno-Orovio et al. [49]; (f) ionic model proposed by Ten Tusscher et al. [48]; (g) parameters controlling diffusion in (63).

\subsubsection{Comprehensive simulation using the new mixed formulations in Sections 5.2.1 and 5.2.1}

In order to address objective O4.I, four different simulations will be carried out in this example. For the four simulations, the passive response is that proposed by Holzapfel-Ogden [21] in (18). The material parameters for this 
model can be found in Fig. $22_{a}$. The values of the parameters controlling diffusion in Eq. (63) are in Fig. 22 . The remaining details of the simulations can be found below and also in Fig. 21.

Simulation-I: the coupling type considered is active stress. The mixed formulation ELEM1-MFA- $T_{a}$ is used. The activation law is that of Nash \& Panfilov [63] (see Fig. 22 ${ }_{b}$ ). The ionic model chosen is the Bueno-Orovio model [49] (see Fig. 22 $2_{e}$.

Simulation-II: the coupling type considered is active stress. The mixed formulation ELEM1-MFA- $T_{a}$ is used in this case. The activation law is that of Wong \& Kuhl [52] (see Fig. 22 c). The ionic model chosen is the Ten Tusscher model [47] (see Fig. $22_{f}$ ).

Simulation-III: the coupling type considered is active strain. The mixed formulation ELEM1-MFA- $\gamma$ is used in this case. The activation law is that of Rossi et al. [7] (see Fig. $22_{d}$ ). The ionic model chosen is the Bueno-Orovio model [49] (see Fig. 22e).

Simulation-IV: the coupling type considered is active strain. The mixed formulation ELEM1-MFA- $\gamma$ is used in this case. The activation law is that of Rossi et al. [7] (see Fig. 22 $d$ ). The ionic model chosen is the Ten Tusscher model [47] (see Fig. $22_{f}$ ).

The number of degrees of freedom for the various simulations is: $\{525705,175235,112088\}$ for $\{\boldsymbol{x}, \phi, p\}$ and $\{4035168,4035168,112088,1345056\}$ for $\{\boldsymbol{F}, \boldsymbol{H}, J, \boldsymbol{A}\}$ and $\left\{\boldsymbol{\Sigma}_{\boldsymbol{F}}, \boldsymbol{\Sigma}_{\boldsymbol{H}}, \Sigma_{J}, \boldsymbol{\Sigma}_{\boldsymbol{A}}\right\}$, where the static condensation procedure in Section 6.2.1 is used in order to condense out the fields $\{\boldsymbol{F}, \boldsymbol{H}, J, \boldsymbol{A}\}$ and $\left\{\boldsymbol{\Sigma}_{\boldsymbol{F}}, \boldsymbol{\Sigma}_{\boldsymbol{H}}, \Sigma_{J}, \boldsymbol{\Sigma}_{\boldsymbol{A}}\right\}$.

Tables 3-4, 5-6, 7-8 and 9-10 display the results obtained for: Simulation-I, Simulation-II, Simulation-III and Simulation-IV (refer to Fig. 21), respectively. The contour plot distribution of relevant variables of interest are plotted for different snapshots of the cardiac cycle. From these pictures it is worth emphasising the similarities in the mechanical contraction obtained from Simulation-I and Simulation-II (Tables 3-4, 5-6). Moreover, the mechanical contraction from Simulation-III and Simulation-IV are very similar (Tables 7-8, 9-10). These similarities are due to the use of the same coupling type in Simulation-I and Simulation-II (active stress in both) and in Simulation-III and Simulation-IV (active strain in both). Even though the ionic model used in Simulation-I and Simulation-II were different (Bueno-Orovio and Ten Tusscher, respectively), there is a very good agreement between the results of both simulations. The same can be said regarding Simulation-III and Simulation-IV. This similarity is due to fact that both ionic models (Bueno-Orovio and Ten Tusscher) have been calibrated to reproduce the cardiac cycle. Hence, provided that the activation type and the activation law are the same, the electrically induced contraction should be reasonably similar.

However, the activation laws for $T_{a}$ (49) (active stress) or $\gamma_{f_{0}}$ (52) (active strain) have not been calibrated to obtain a similar electrically induced mechanical response to the best of the authors' knowledge. In our study, we have limited to use the material parameters reported in the available literature for the three activation laws in Figs. $22_{b}-22_{d}$ considered. This explains the dissimilarities between Simulation-I (or Simulation-II) and Simulation-III (or Simulation-IV). 
Table 3

Numerical example 4.1. Simulation-I (activation type: active stress; ionic model: Bueno-Ovorio; activation law: Nash \& Panfilov; mixed formulation: ELEM1-MFA- $T_{a}$ ). Snapshots for time $t=\{150,200,320,1000\}$ (ms) of the cardiac cycle. Contour plot of $\phi(\mathrm{mV})$, cardiomyocite stress $T_{a}(\mathrm{~Pa})$, right Cauchy-Green tensor component $C_{X X}$ and three internal variables, namely $\{v, w, s\}$.

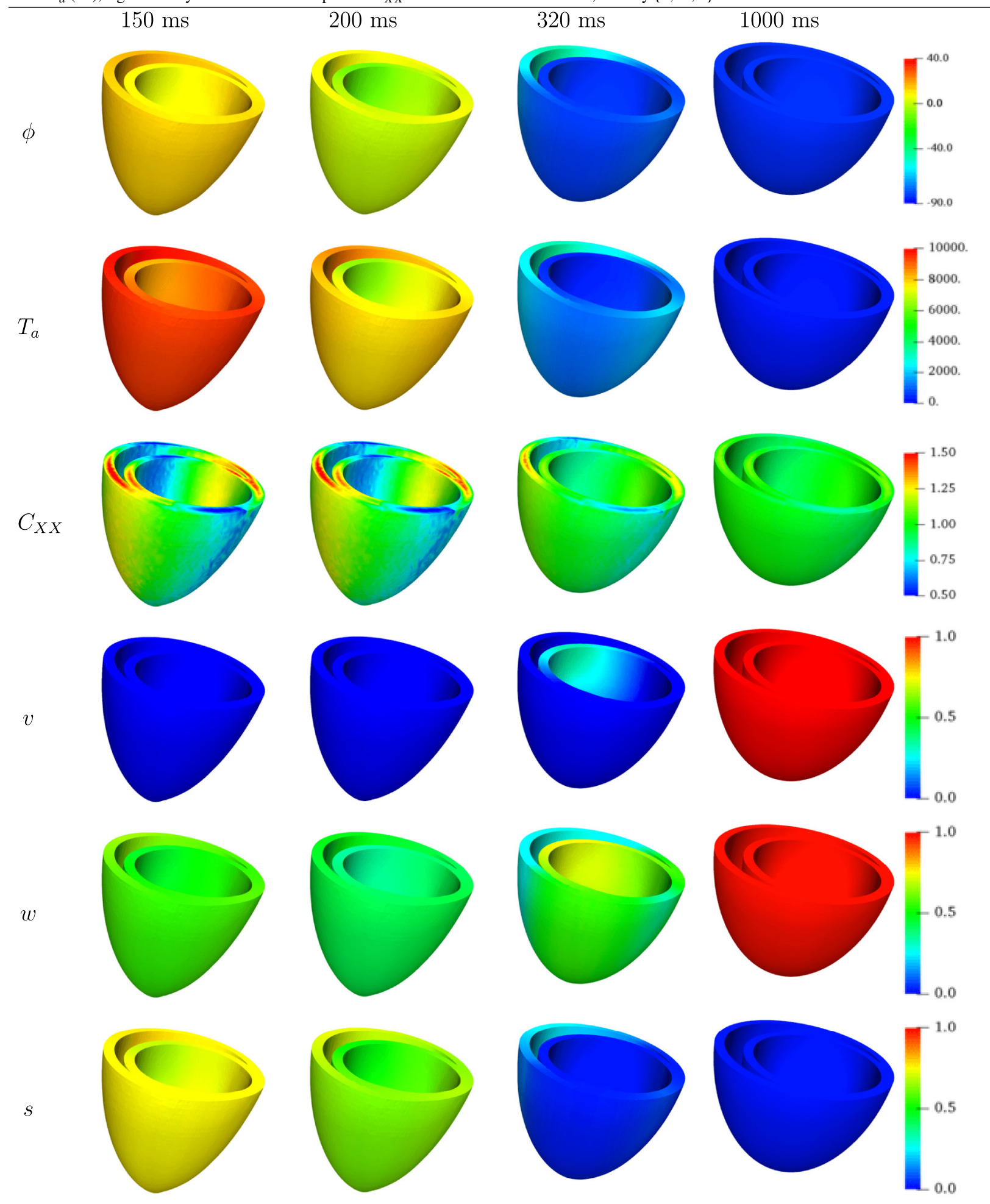


Table 4

Numerical example 4.1. Simulation-I (activation type: active stress; ionic model: Bueno-Ovorio; activation law: Nash \& Panfilov; mixed formulation: ELEM1-MFA- $T_{a}$ ). Snapshots for time $t=\{5,20,40,100\}(\mathrm{ms})$ of the cardiac cycle. Contour plot of $\phi(\mathrm{mV})$, cardiomyocite stress $T_{a}(\mathrm{~Pa})$, right Cauchy-Green tensor component $C_{X X}$ and three internal variables, namely $\{v, w, s\}$.
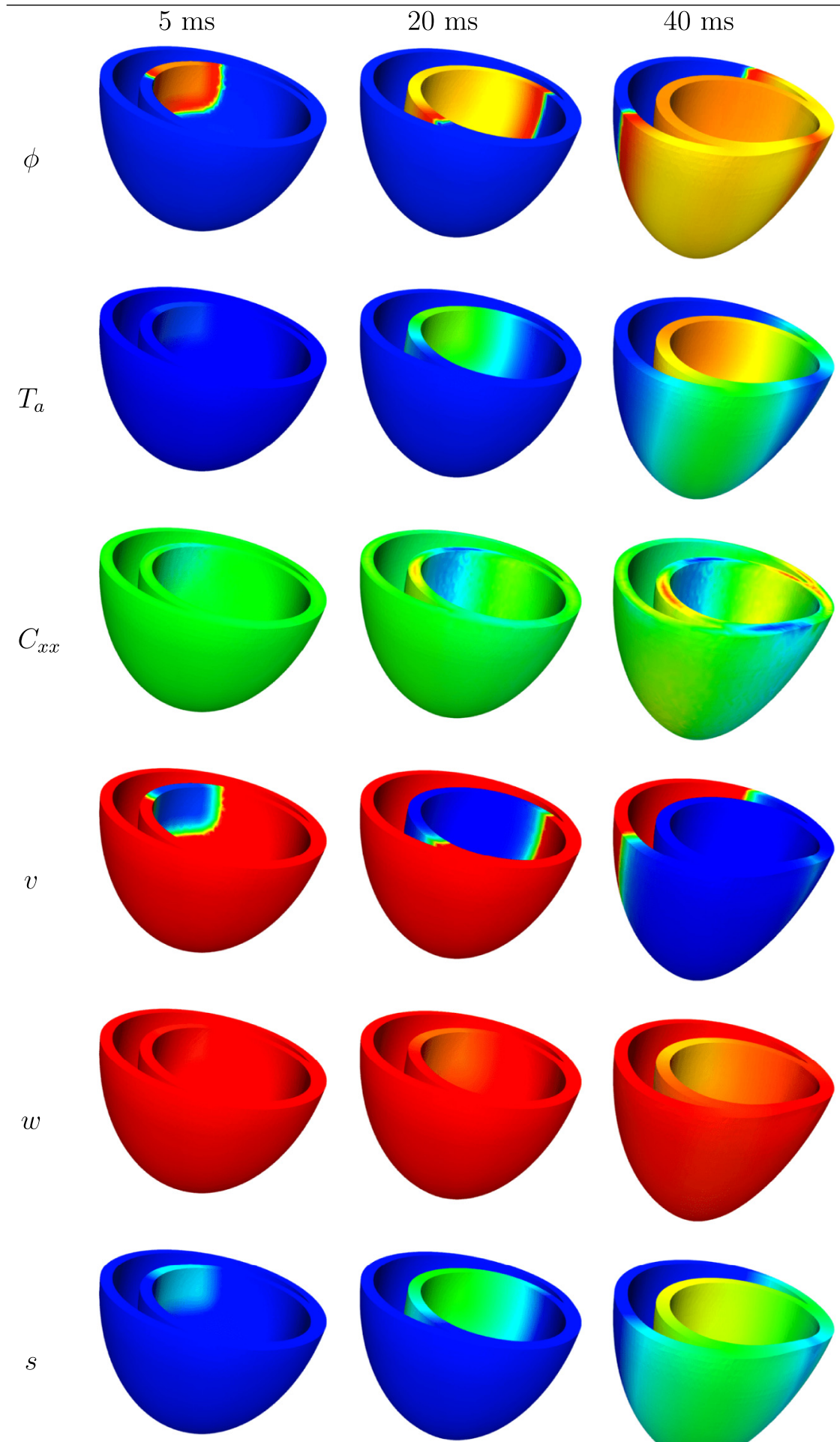
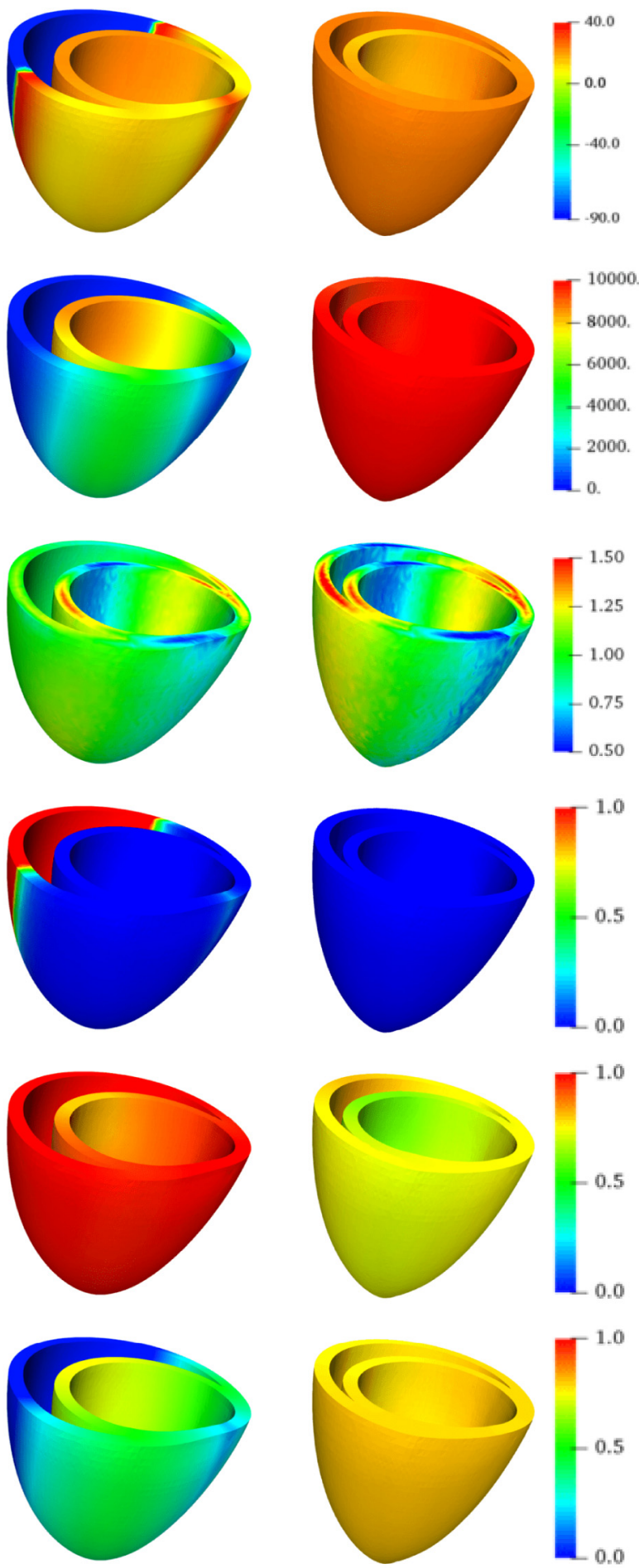
Table 5

Numerical example 4.1. Results for Simulation-II (activation type: active stress; ionic model: Ten Tusscher; activation law: Wong \& Kuhl; mixed formulation: ELEM1-MFA- $T_{a}$ ). Snapshots for time $t=\{5,20,40,100\}$ (ms) of the cardiac cycle. Contour plot of $\phi$ (mV), cardiomyocite stress $T_{a}(\mathrm{~Pa})$, right Cauchy-Green tensor component $C_{X X}$ and three ionic concentrations, namely, $\left\{\mathrm{Ca}^{++}, \mathrm{Na}^{+}, \mathrm{K}^{+}\right\}$(units of $\mathrm{mM}$ ).
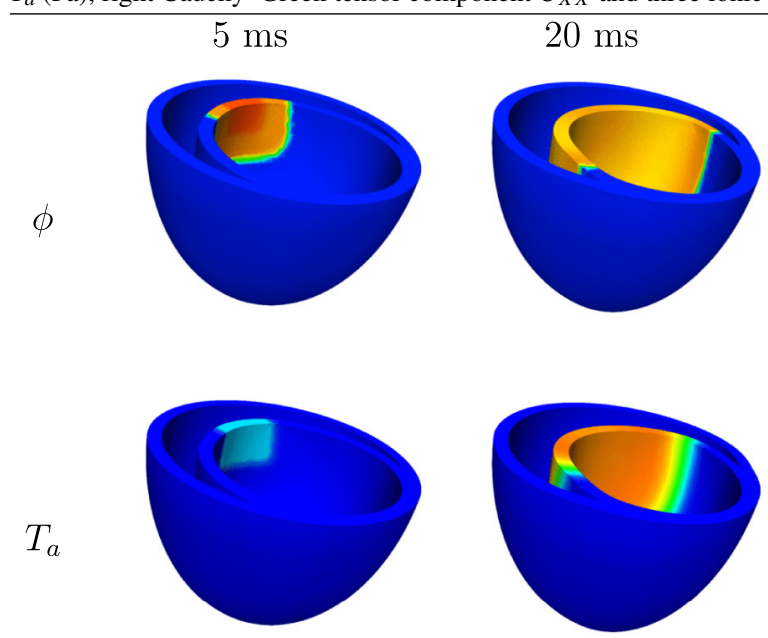

$T_{a}$
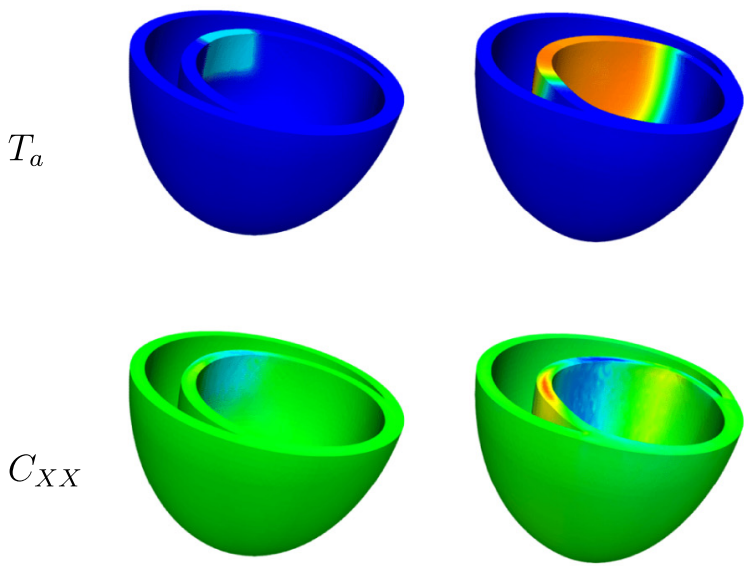

$C_{X X}$
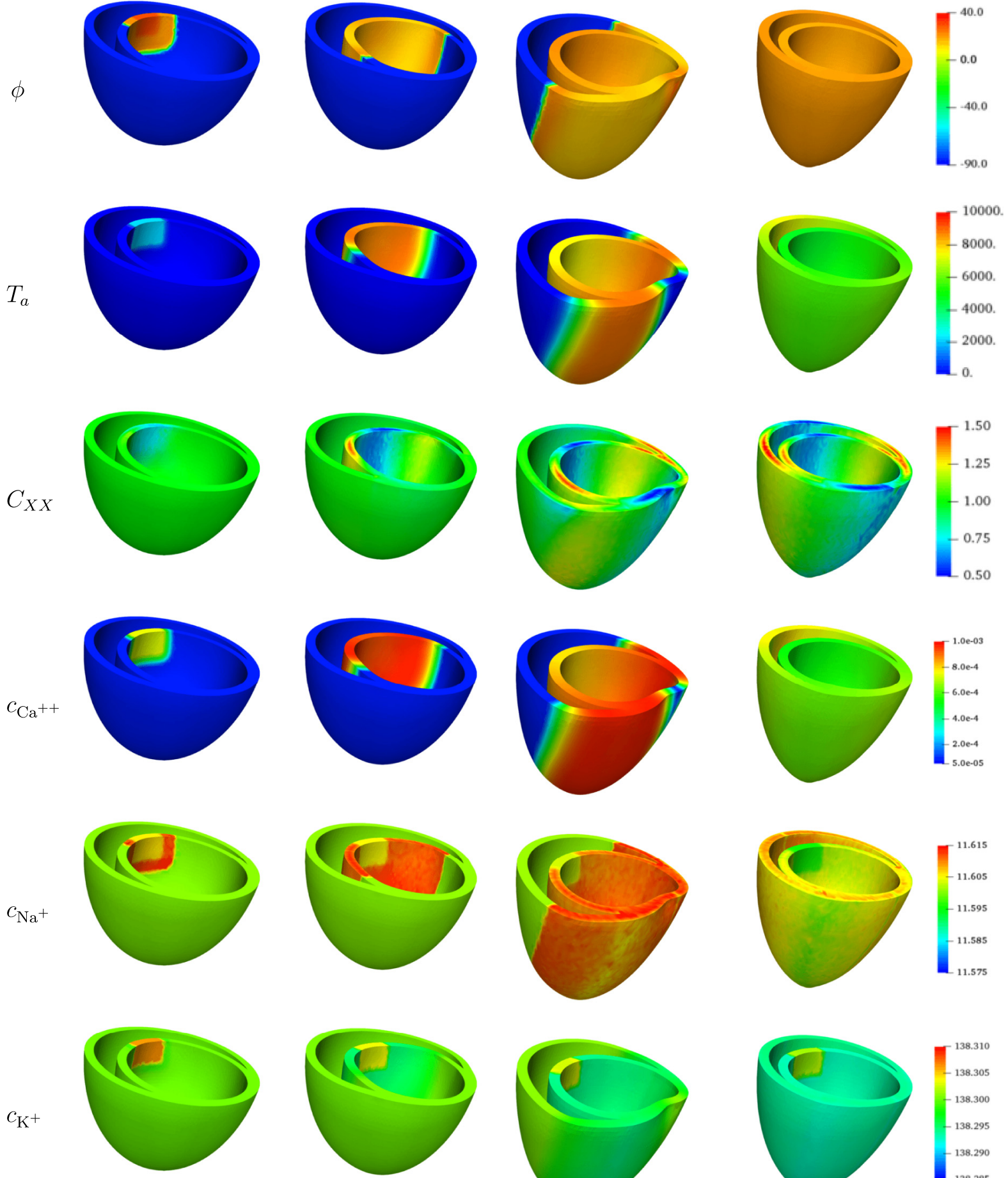

$c_{\mathrm{Na}^{+}}$

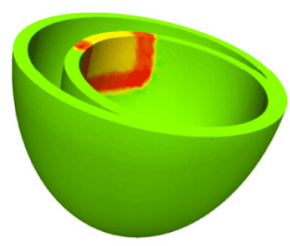

$c_{\mathrm{K}^{+}}$
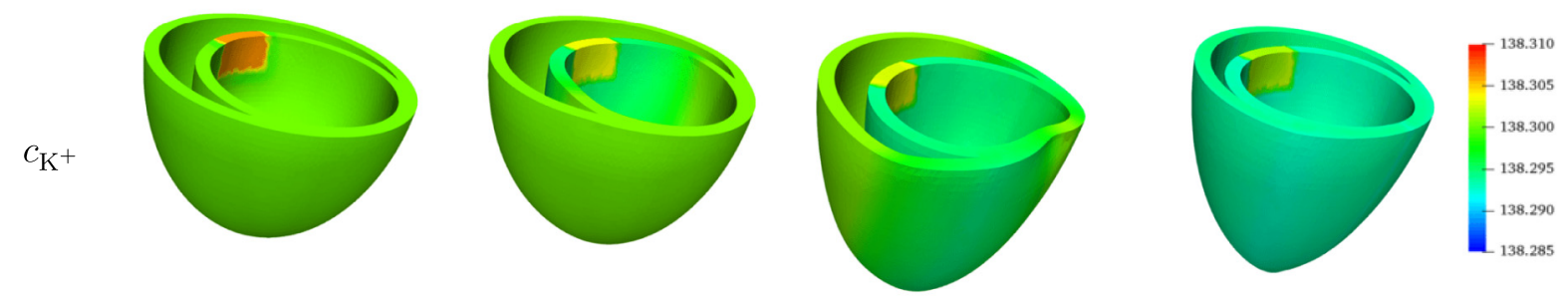
Table 6

Numerical example 4.1. Results for Simulation-II (activation type: active stress; ionic model: Ten Tusscher; activation law: Wong \& Kuhl; mixed formulation: ELEM1-MFA- $T_{a}$ ). Snapshots for time $t=\{150,200,320,1000\}$ (ms) of the cardiac cycle. Contour plot of $\phi$ (mV), cardiomyocite stress $T_{a}(\mathrm{~Pa})$, right Cauchy-Green tensor component $C_{X X}$ and three ionic concentrations, namely, $\left\{\mathrm{Ca}^{++}, \mathrm{Na}^{+}, \mathrm{K}^{+}\right\}$(units of mM).

$\phi$
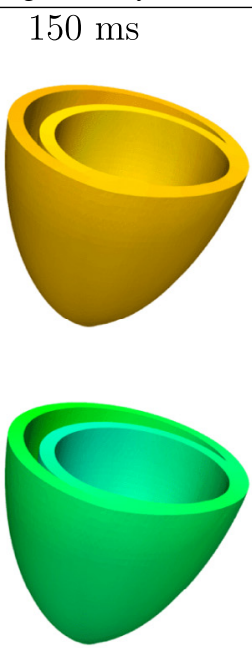

$C_{X X}$

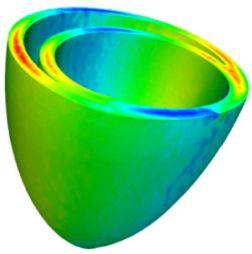

$c_{\mathrm{Ca}^{++}}$

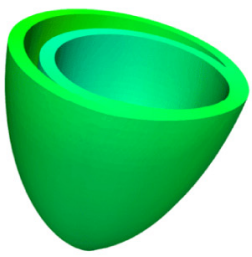

$c_{\mathrm{Na}^{+}}$
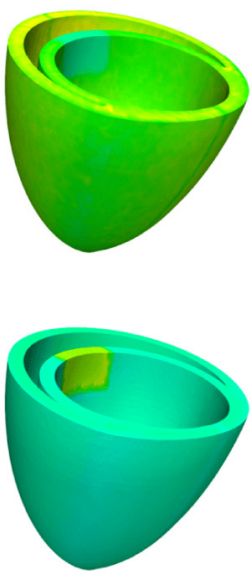

\section{$200 \mathrm{~ms}$}
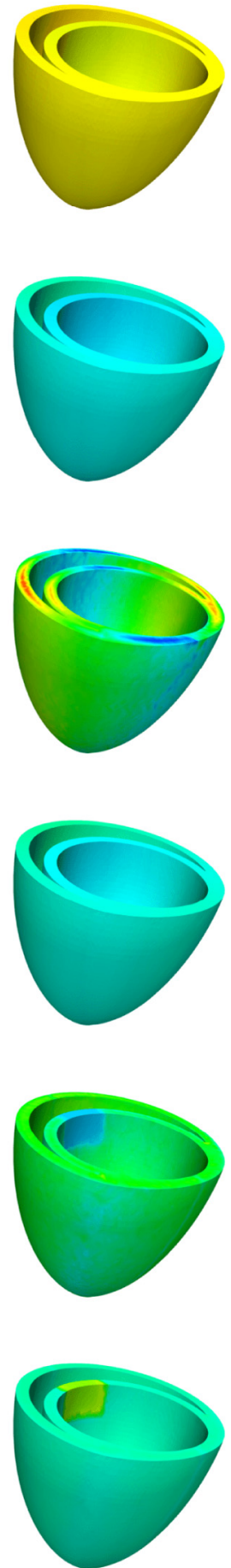
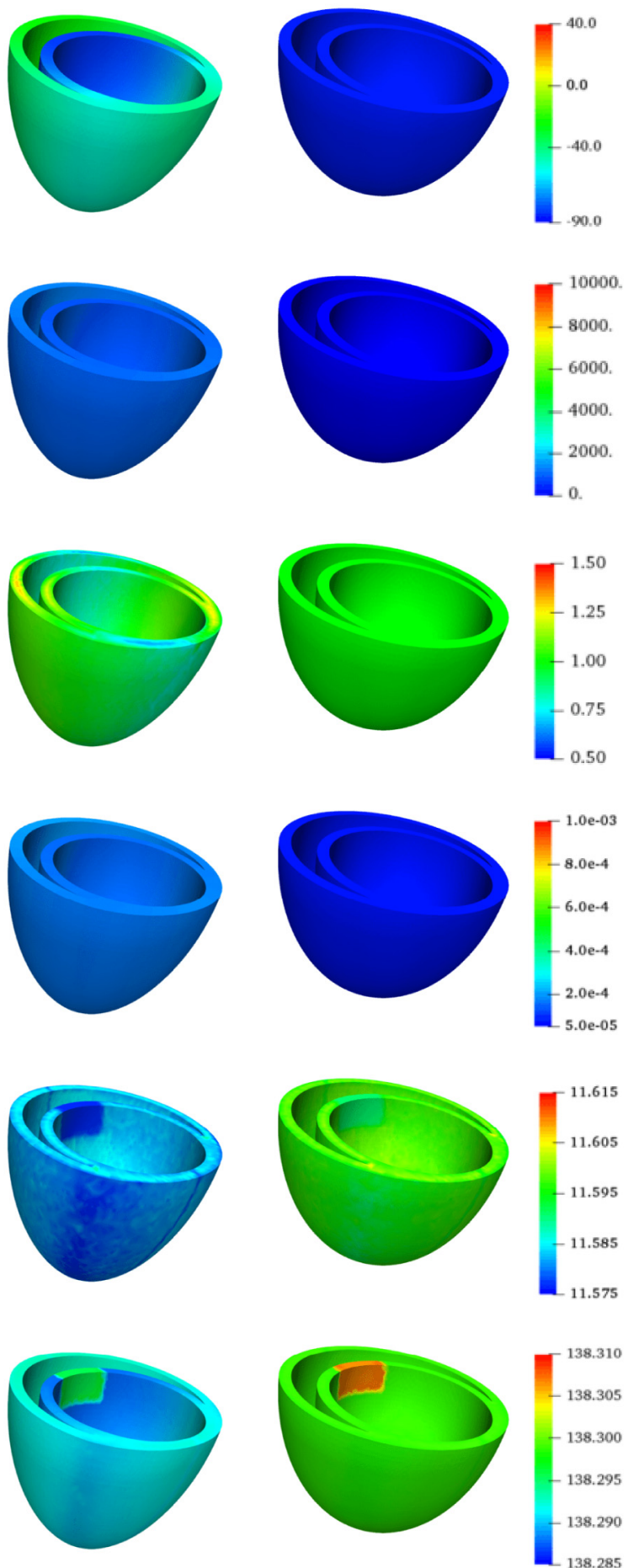
Table 7

Numerical example 4.1. Simulation-III (activation type: active strain; ionic model: Bueno-Ovorio; activation law: Rossi et al.; mixed formulation: ELEM1-MFA- $\gamma$ ). Snapshots for time $t=\{5,20,40,100\}(\mathrm{ms})$ of the cardiac cycle. Contour plot of $\phi(\mathrm{mV})$, electrical stretch $\gamma_{f_{0}}$, right CauchyGreen tensor component $C_{X X}$ and three internal variables, namely $\{v, w, s\}$.

$20 \mathrm{~ms}$

$\phi$
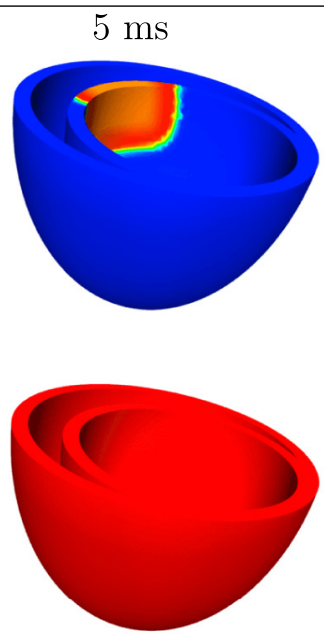

$C_{X X}$

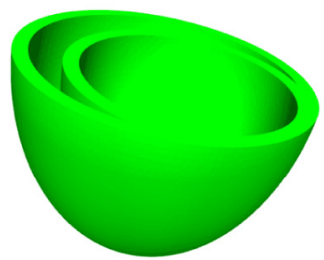

$v$

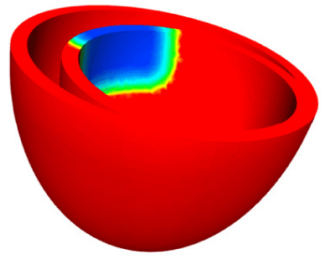

$w$
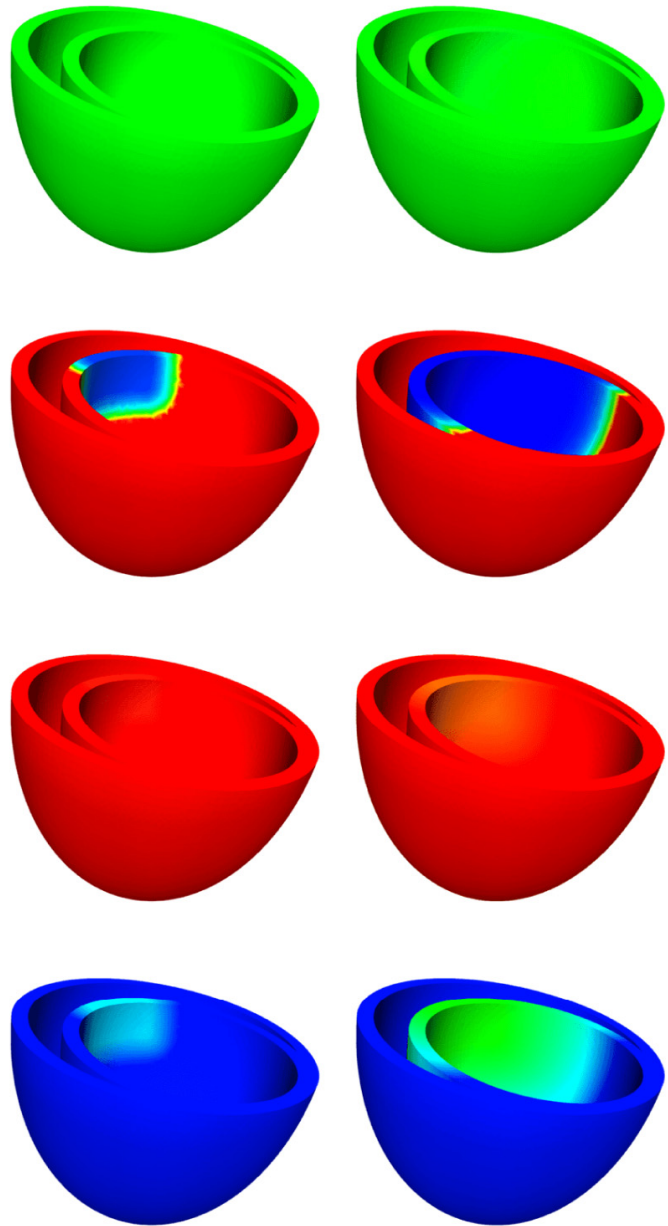
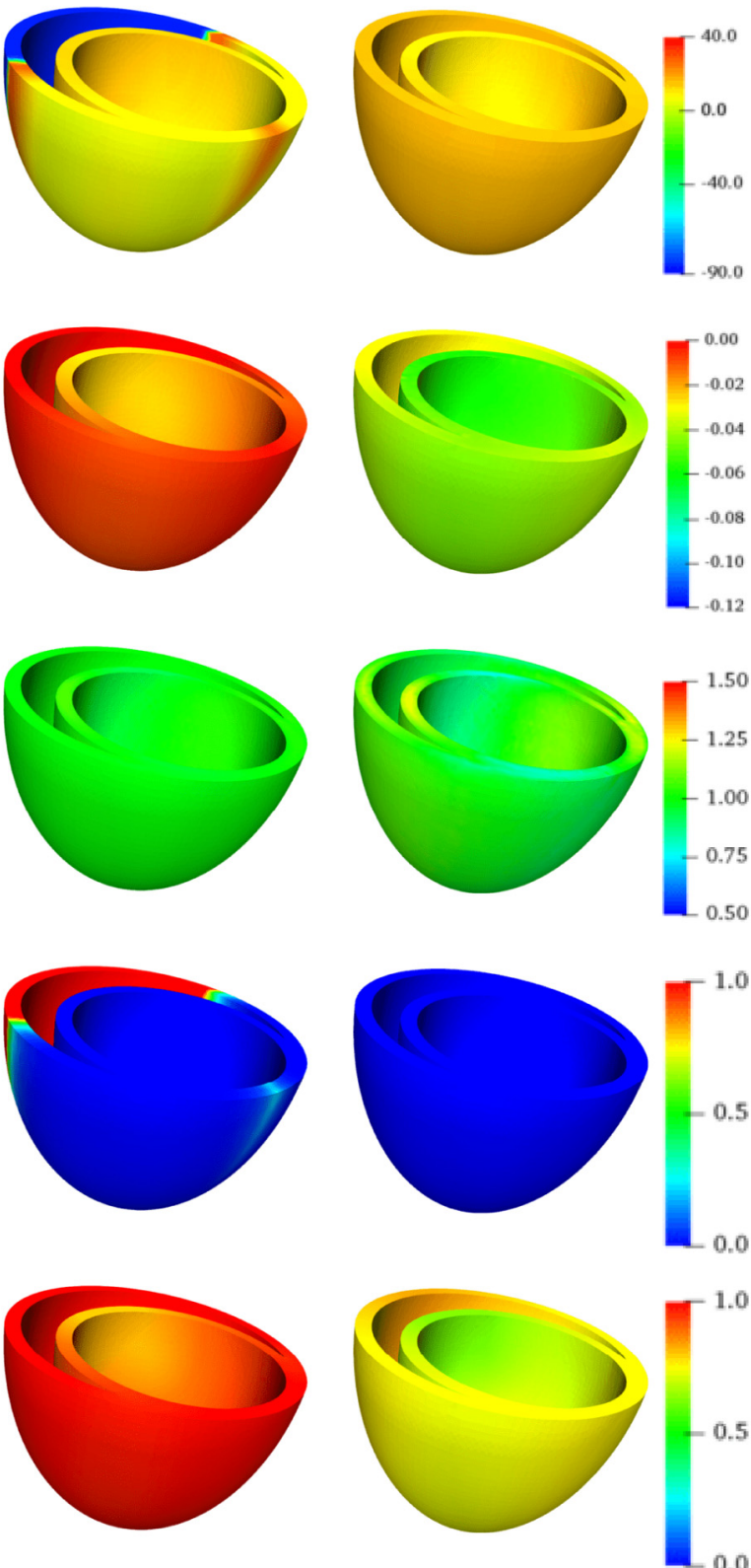

0.0

1.0

0.0
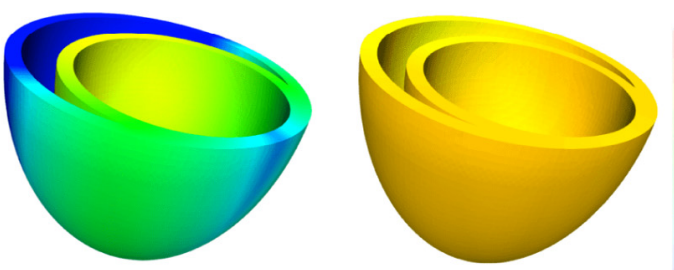
Table 8

Numerical example 4.1. Simulation-III (activation type: active strain; ionic model: Bueno-Ovorio; activation law: Rossi et al.; mixed formulation: ELEM1-MFA- $\gamma$ ). Snapshots for time $t=\{150,200,320,1000\}(\mathrm{ms})$ of the cardiac cycle. Contour plot of $\phi(\mathrm{mV})$, electrical stretch $\gamma_{f_{0}}(\mathrm{~Pa})$, right Cauchy-Green tensor component $C_{X X}$ and three internal variables, namely $\{v, w, s\}$.

Cauchy-Green tensor component $C_{X X}$ and three internal wariables, namely $\{v, w, s\}$.
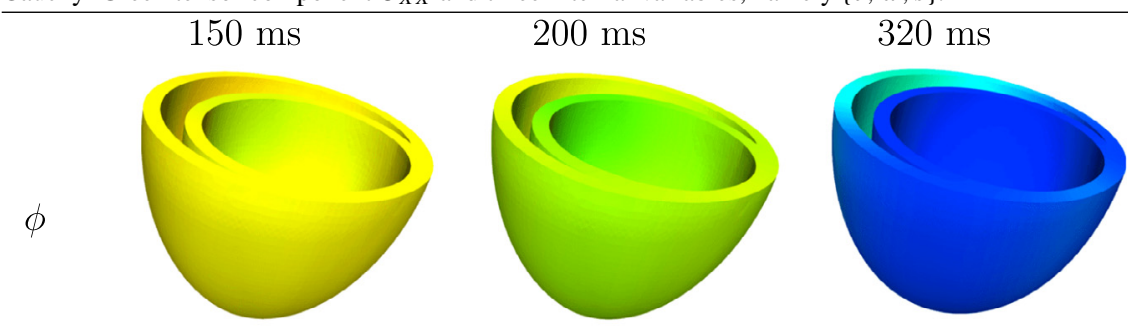

\section{$1000 \mathrm{~ms}$}
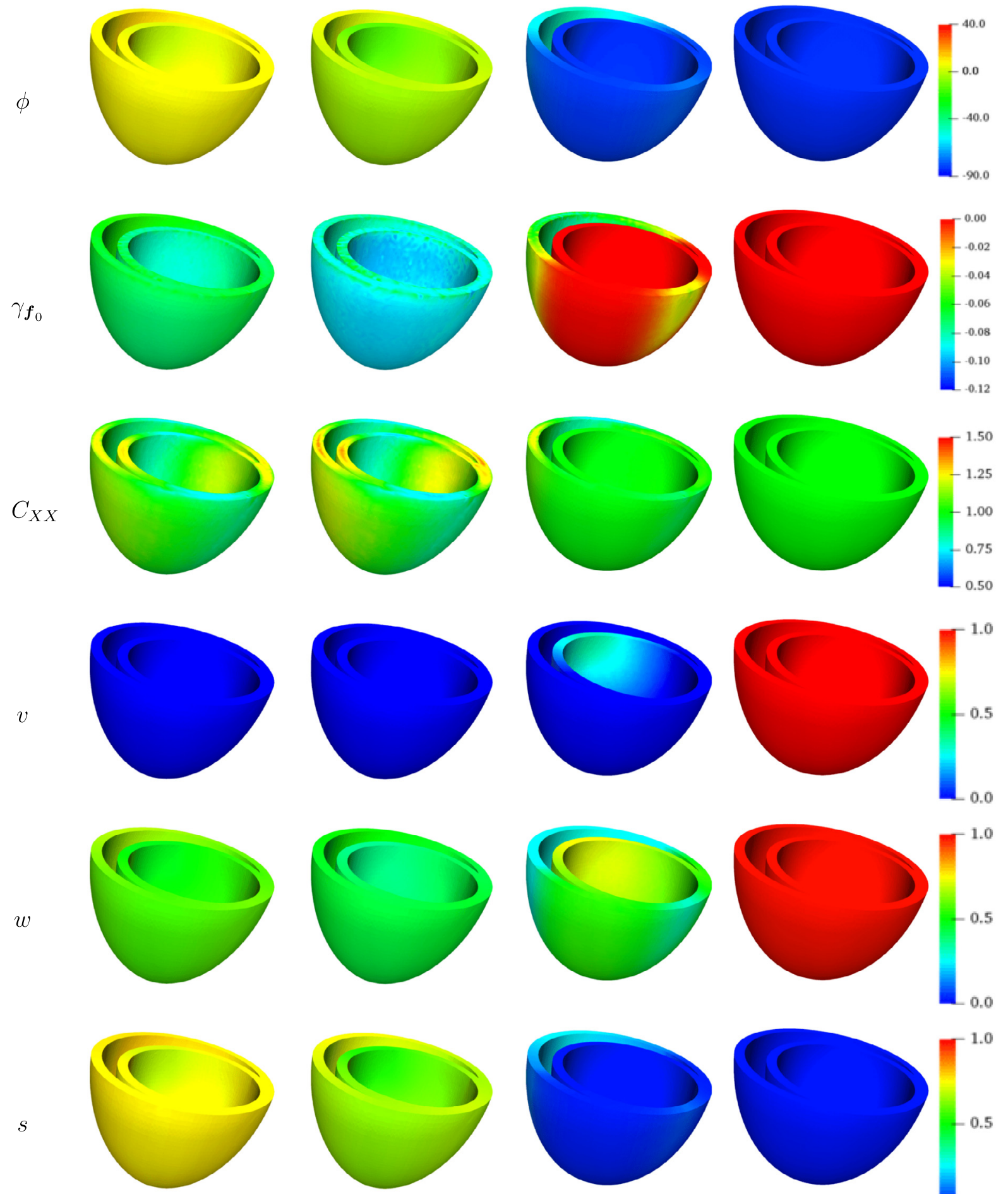
Table 9

Numerical example 4.1. Results for Simulation-IV (activation type: active strain; ionic model: Ten Tusscher; activation law: Rossi et al.; mixed formulation: ELEM1-MFA- $\gamma$ ). Snapshots for time $t=\{5,20,40,100\}$ (ms) of the cardiac cycle. Contour plot of $\phi(\mathrm{mV})$, electrical stretch $\gamma_{f_{0}}$, right Cauchy-Green tensor component $C_{X X}$ and three ionic concentrations, namely, $\left\{\mathrm{Ca}^{++}, \mathrm{Na}^{+}, \mathrm{K}^{+}\right\}$(units of $\mathrm{mM}$ ).

$\phi$
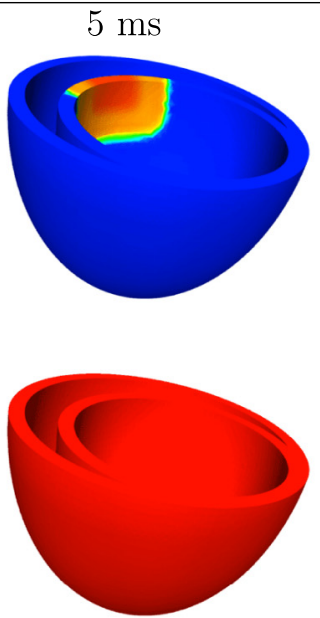

$C_{X X}$
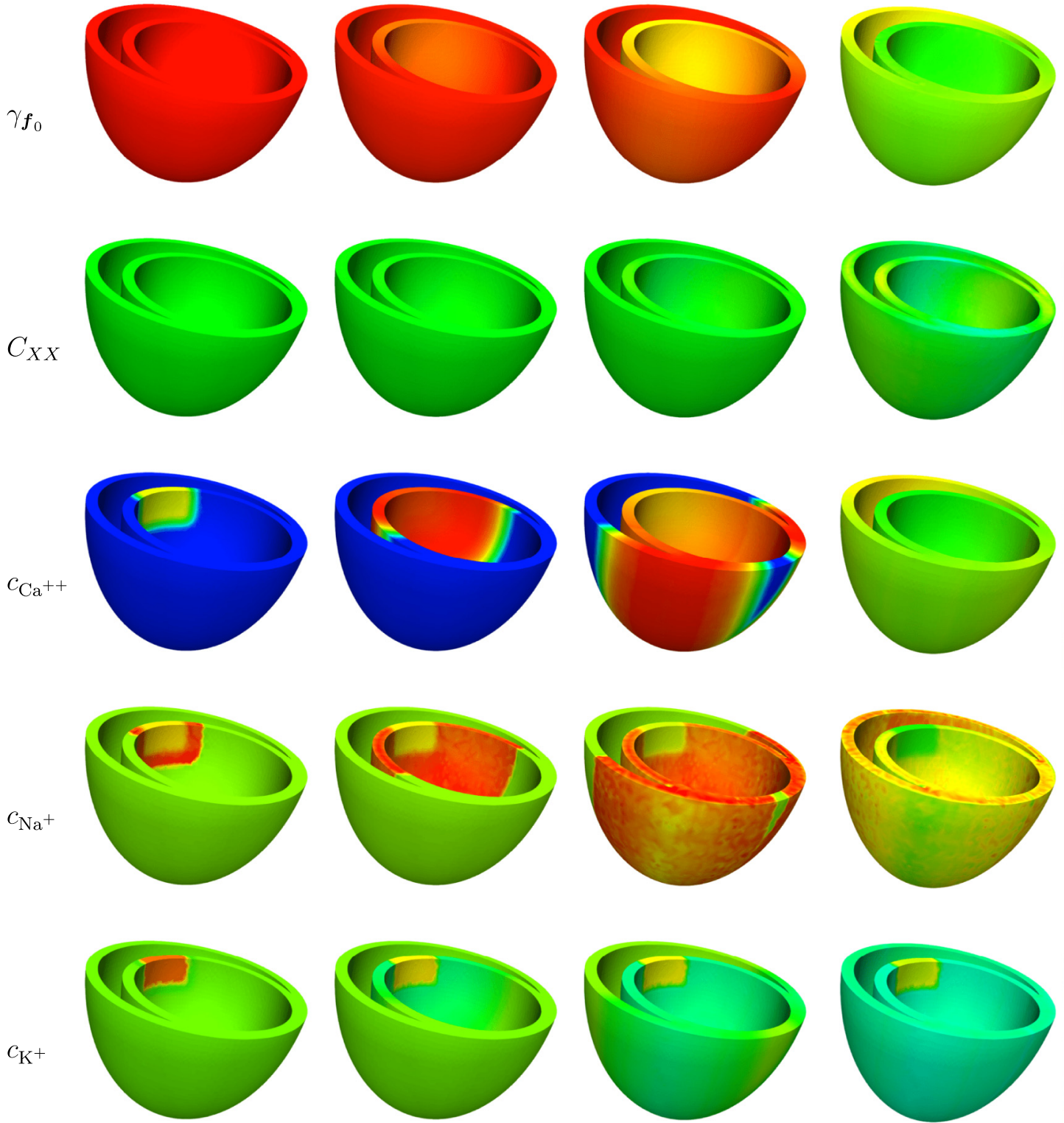
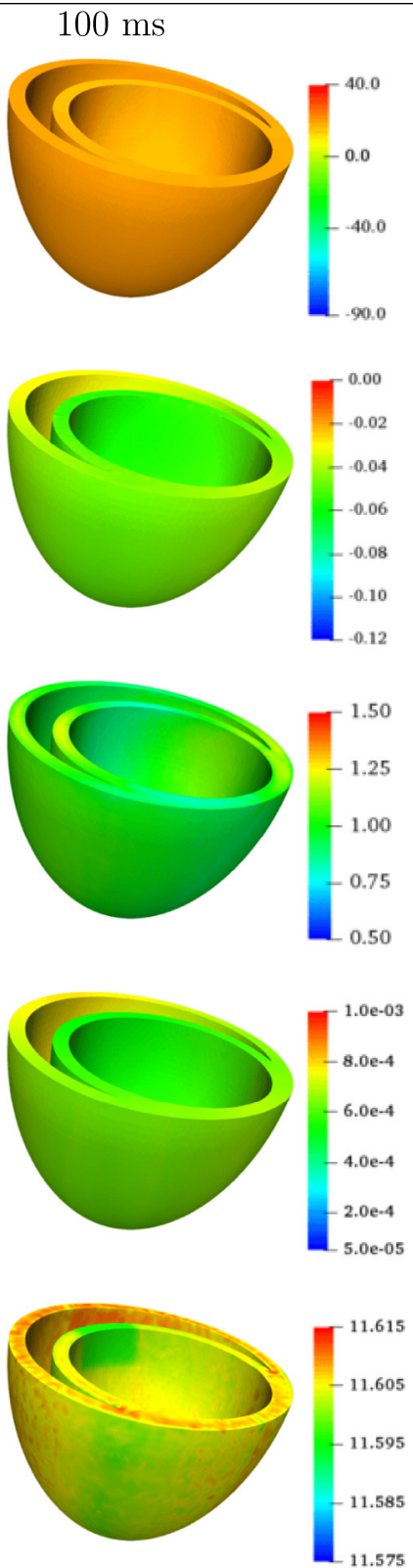

.0 .12

\section{e-4 \\ $0 \mathrm{e}-4$ \\ $.0 \mathrm{e}-4$ \\ $5.0 \mathrm{e}-05$}
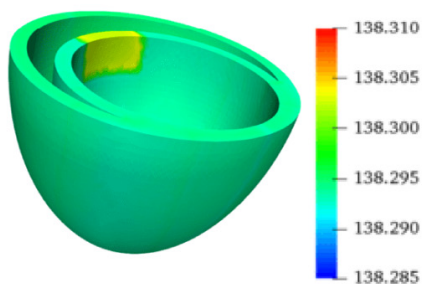
Table 10

Numerical example 4.1. Results for Simulation-IV (activation type: active strain; ionic model: Ten Tusscher; activation law: Rossi et al.; mixed formulation: ELEM1-MFA- $\gamma$ ). Snapshots for time $t=\{150,200,320,1000\}$ (ms) of the cardiac cycle. Contour plot of $\phi(\mathrm{mV})$, electrical stretch $\gamma_{f_{0}}$, right Cauchy-Green tensor component $C_{X X}$ and three ionic concentrations, namely, $\left\{\mathrm{Ca}^{++}, \mathrm{Na}^{+}, \mathrm{K}^{+}\right\}$(units of $\mathrm{mM}$ ).
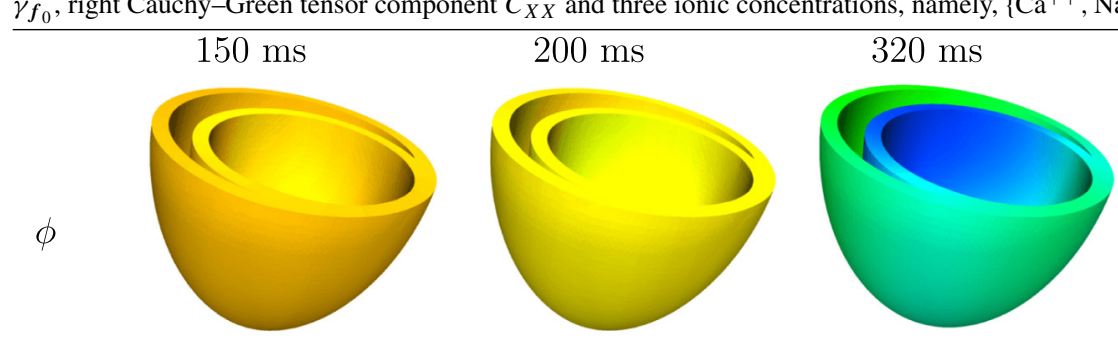

$1000 \mathrm{~ms}$
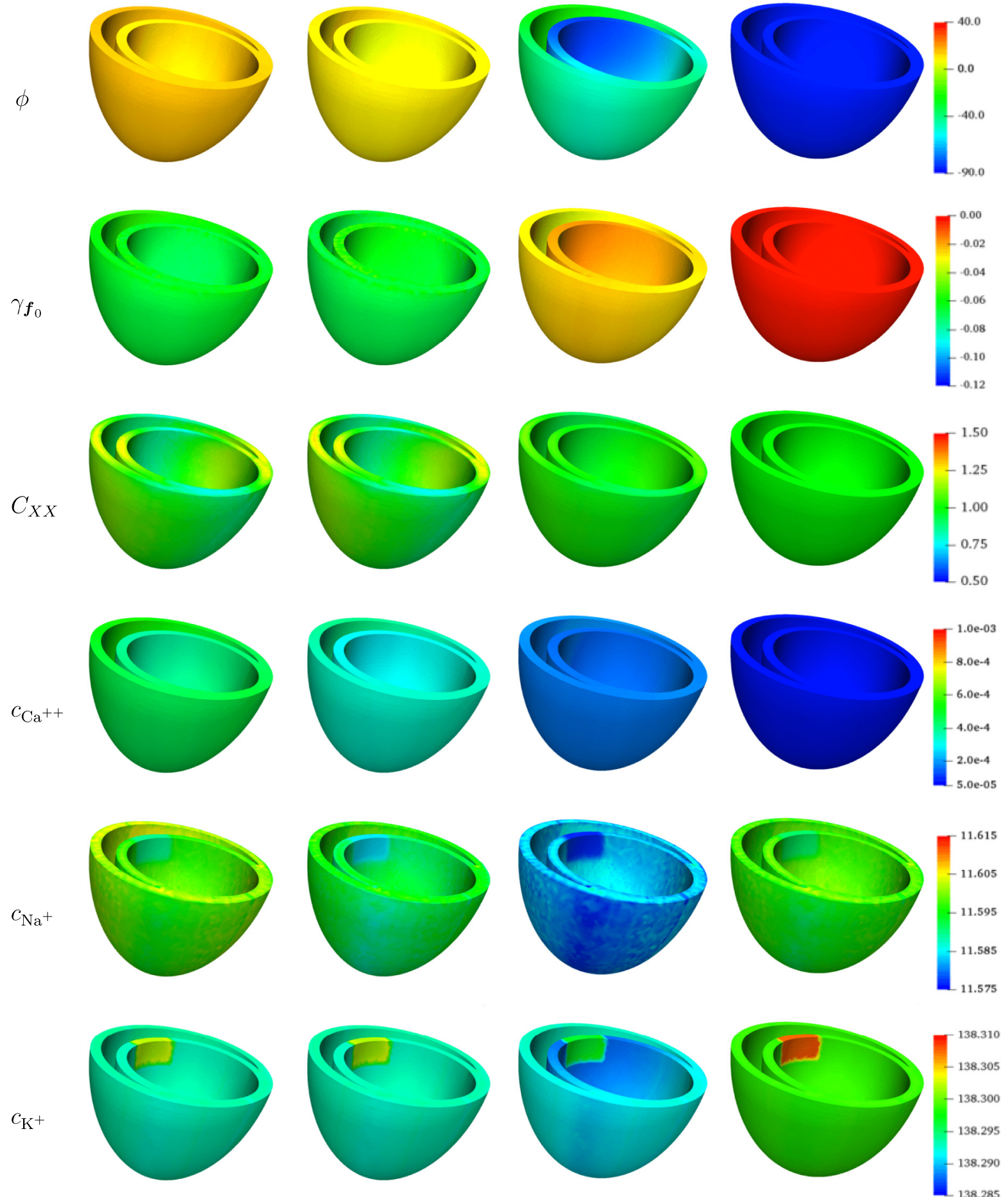


\section{Table 11}

Numerical example 4.2. Activation type: active stress; ionic model: Bueno-Orovio; activation law: Nash \& Panfilov. Snapshots for time $t=$ $\{20,40,100,200\}$ (ms) of the cardiac cycle. Contour plot of right Cauchy-Green tensor component $C_{X X}$ colour-scaled to [0.5,1.5]. Results for ELEM1-MFA- $T_{a}$ and $P_{1}-P_{1}$ formulations.

$\begin{array}{llll}P_{1}-P_{1} & \text { MFA- } T_{a} & P_{1}-P_{1} & \text { MFA- } T_{a}\end{array}$

$5 \mathrm{~ms}$
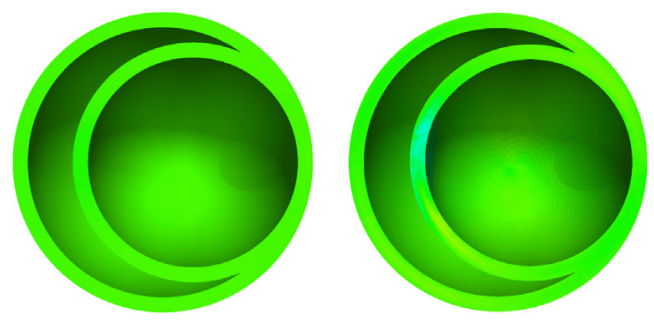

$20 \mathrm{~ms}$
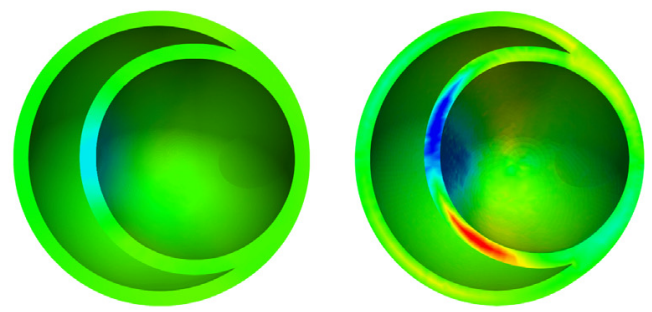

$40 \mathrm{~ms}$
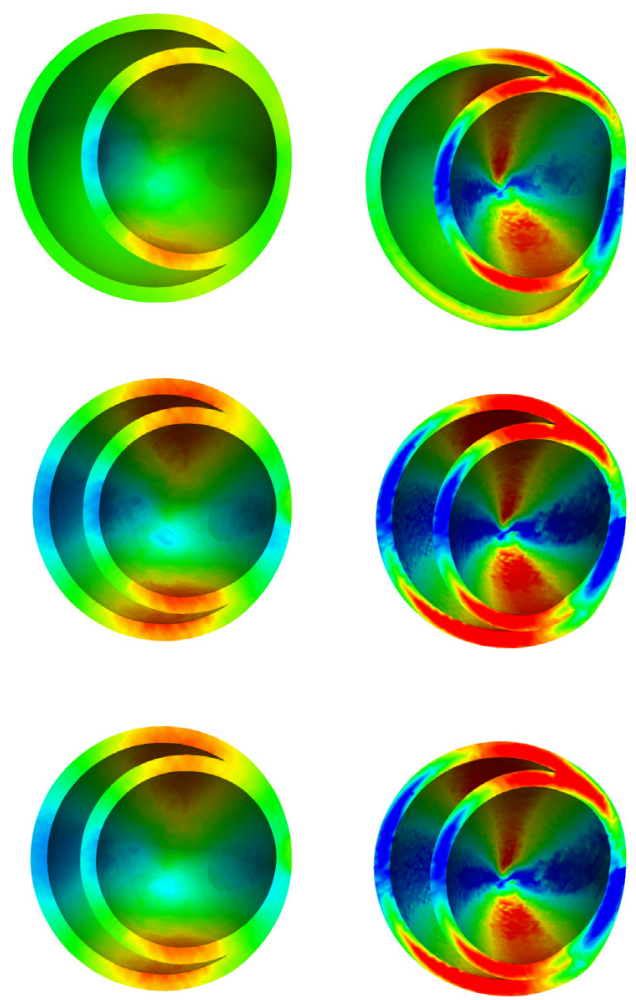
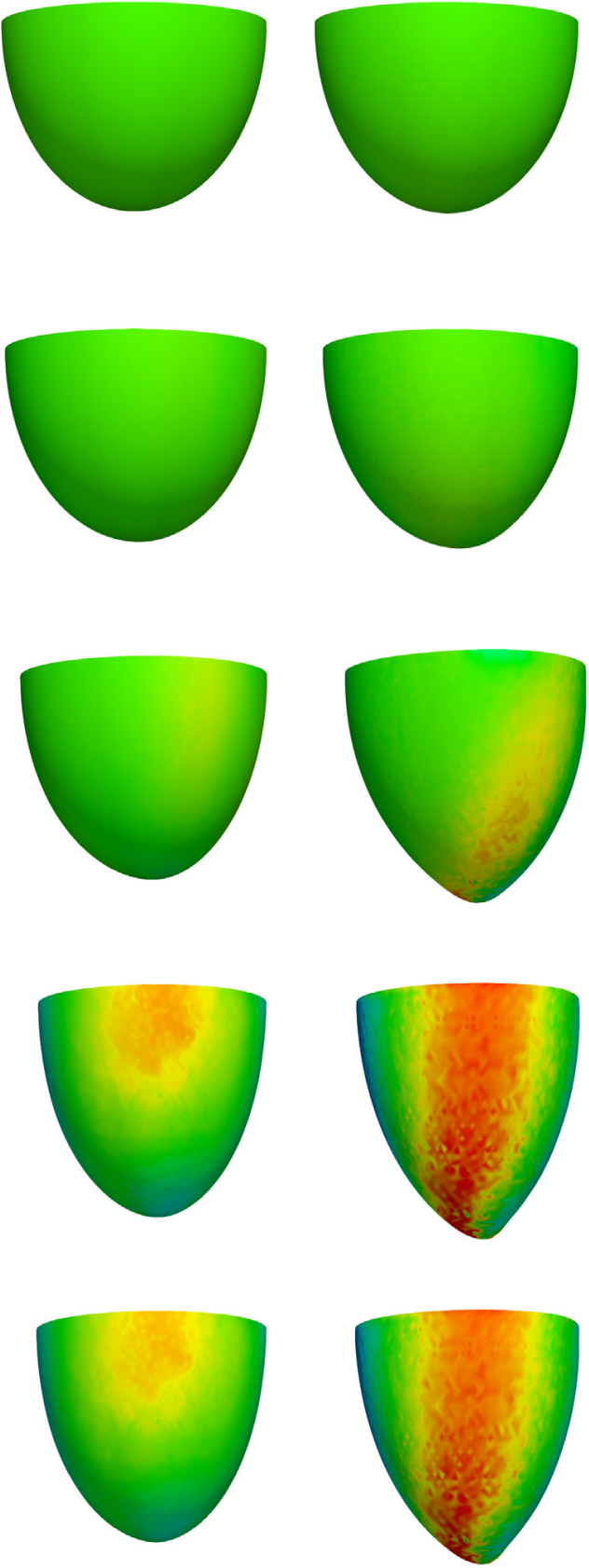
Table 12

Numerical example 4.3. Simulation details: activation type: active stress; ionic model: Bueno-Orovio; activation law: Nash \& Panfilov; mixed formulation: ELEM1-MFA- $T_{a}$. Snapshots for time $t=\{2,20,40\}(\mathrm{ms})$ of the cardiac cycle. Ellipticity indicator $q$ at a fixed Gauss point for angles $\alpha$ and $\beta$ when considering Holzapfel-Ogden law in the case of: (upper row) with Macaulay brackets and (lower row) without Macaulay brackets. Loss of ellipticity in the later case.
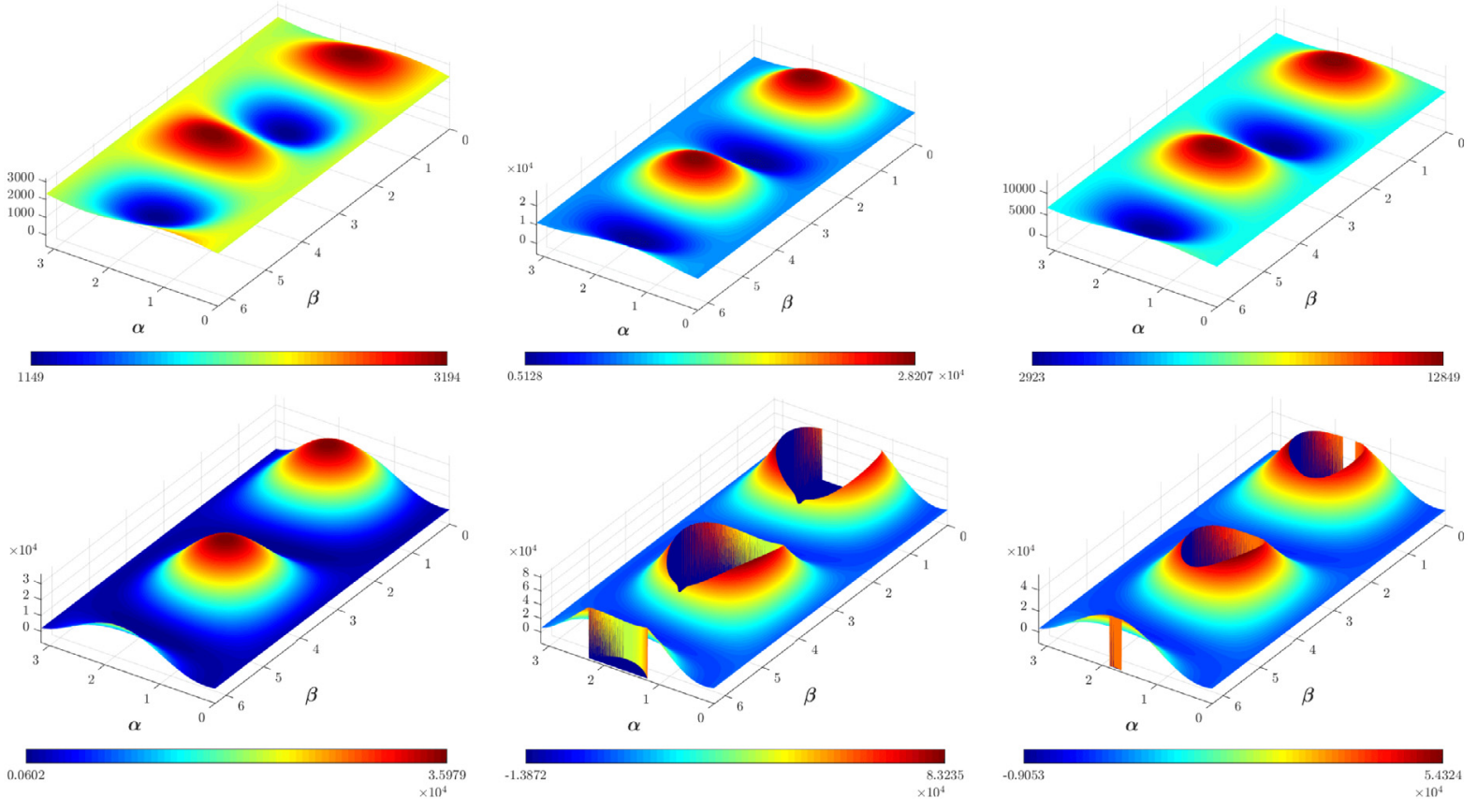

\subsection{Numerical example 4.2: comparison against $P_{1}-P_{1}$ element}

This example addresses objective O4.II. We model in this example the cardiac cycle considering an active stress approach, the Nash \& Panfilov [63] activation law for $T_{a}$ and the Bueno-Orovio [49] ionic model. For the passive response we use Holzapfel-Ogden model (18). The relevant material parameters can be found in Fig. 22.

We then compare the ELEM1-MFA- $T_{a}$ formulation against a conventional formulation where the unknown fields are the geometry $\boldsymbol{x}$ and the transmembrane potential $\phi$. Truly incompressibility is not enforced in this formulation. Instead, nearly incompressibility is enforced by means of high value for $\kappa$ in the volumetric functional $U\left(J_{x}\right)$ in $(18)$. In this formulation, $\{\boldsymbol{x}-\phi\}$ are interpolated using linear tetrahedral Finite Elements. We denote this formulation as $P_{1}-P_{1}$.

The number of degrees of freedom for the ELEM1-MFA- $T_{a}$ is: $\{525705,175235,112088\}$ for $\{\boldsymbol{x}, \phi, p\}$ and $\{4035168,4035168,112088,1345056\}$ for $\{\boldsymbol{F}, \boldsymbol{H}, J, \boldsymbol{A}\}$ and $\left\{\boldsymbol{\Sigma}_{\boldsymbol{F}}, \boldsymbol{\Sigma}_{\boldsymbol{H}}, \Sigma_{J}, \boldsymbol{\Sigma}_{\boldsymbol{A}}\right\}$, where the static condensation procedure in Section 6.2.1 is used in order to condense out the fields $\{\boldsymbol{F}, \boldsymbol{H}, J, \boldsymbol{A}\}$ and $\left\{\boldsymbol{\Sigma}_{\boldsymbol{F}}, \boldsymbol{\Sigma}_{\boldsymbol{H}}, \Sigma_{J}, \boldsymbol{\Sigma}_{\boldsymbol{A}}\right\}$. For the $P_{1}-P_{1}$ formulation we use a discretisation with a number of degrees of freedom of $\{775668,258556\}$ for $\{\boldsymbol{x}, \phi\}$. Our objective is to compare both formulations for a similar number of degrees of freedom for $\{\boldsymbol{x}, \phi\}$ for both ELEM1MFA- $T_{a}$ and $P_{1}-P_{1}$ formulations.

Table 11 shows the severe locking obtained with the $P_{1}-P_{1}$ formulation. A considerably smaller electrically induced contraction of the ventricles is obtained with this formulation in comparison to the ELEM1-MFA- $T_{a}$ formulation. These results enable to conclude that the electrically induced deformation obtained by means of the $P_{1}-P_{1}$ is not reliable and hence, this formulation should not be used in this context.

\subsection{Numerical example 4.3: analysis of possible loss of ellipticity of passive response}

This example addresses objective O4.III. The susceptibility of Holzapfel-Ogden model (18) for the passive response of the myocardium is analysed when considering both active strain and active stress approaches. In addition, 
Table 13

Numerical example 4.3. Simulation details: activation type: active strain; ionic model: Bueno-Orovio; activation law: Rossi et al.; mixed formulation: ELEM1-MFA- $T_{a}$. Snapshots for time $t=\{2,20,40\}(\mathrm{ms})$ of the cardiac cycle. Ellipticity indicator $q$ at a fixed Gauss point for angles $\alpha$ and $\beta$ when considering Holzapfel-Ogden law in the case of: (upper row) with Macaulay brackets and (lower row) without Macaulay brackets. Loss of ellipticity in the later case.
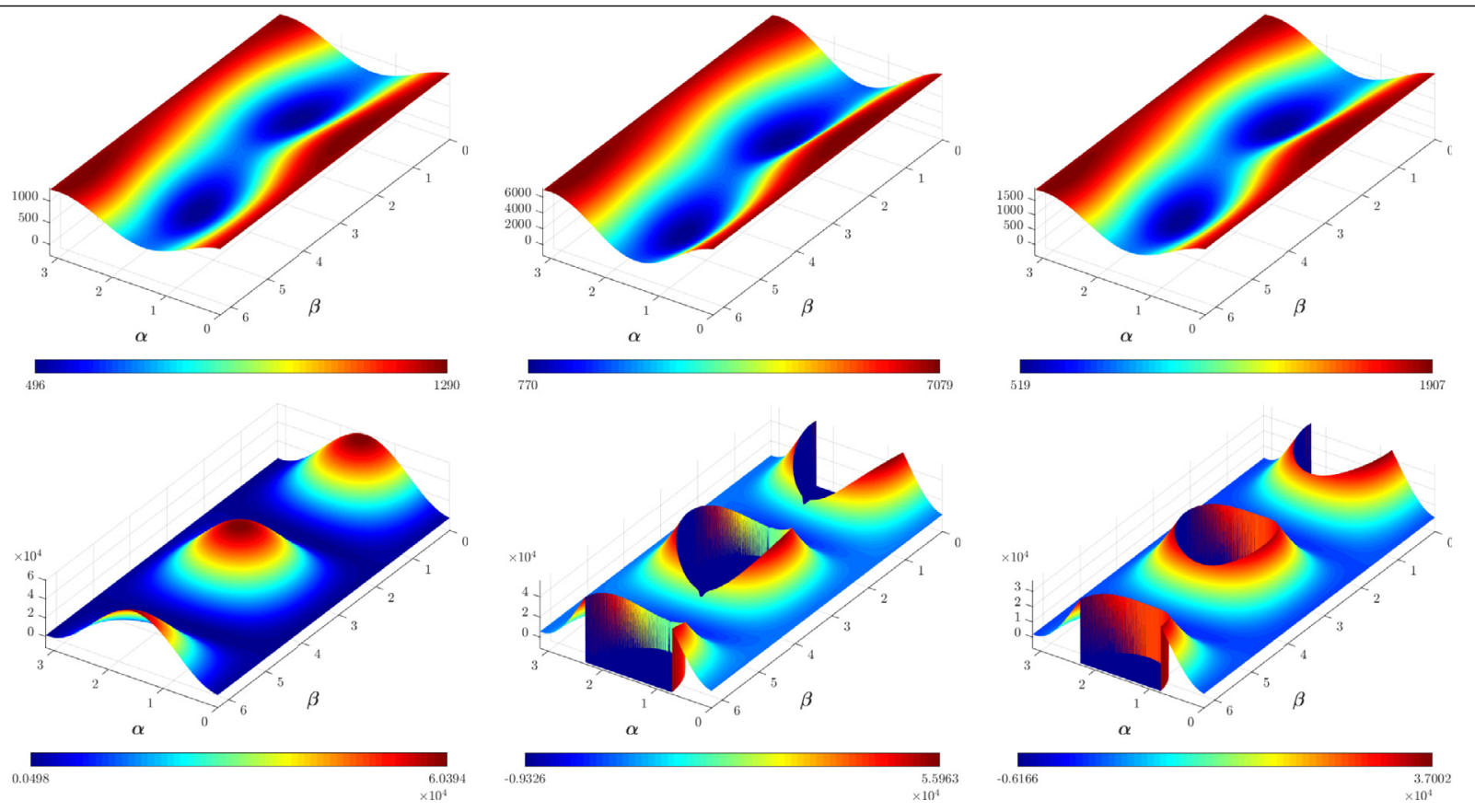

the influence of the consideration of the Macauly brackets in Eq. (18) upon the possible loss of ellipticity of this model is also studied. Mathematically, the consideration of these brackets implies that the energetic contributions in $W^{\text {ani }}$ associated with the fibres $\left\{f_{0}, s_{0}\right\}$ are only active when the invariants $I_{4, f_{0}}$ and $I_{4, s_{0}}$ are positive. Physically, this means that the fibres contribute to the overall passive response when they are tractioned. On the contrary, not considering the Macaulay brackets implies assuming that these fibres contribute to the overall response even when they are compressed (i.e. when $I_{4, f_{0}}$ and $I_{4, s_{0}}$ are negative).

In order to analyse the possible loss of ellipticity, we study the positive definiteness of the acoustic tensor $\mathcal{C}_{V V}$ in Eq. (16) for any direction $\boldsymbol{V}$. For any direction $\boldsymbol{V}$, spherically parametrised in terms of the angles $0 \leq \alpha \leq \pi$ and $0 \leq \beta \leq 2 \pi$, i.e. $\boldsymbol{V}=\left[\begin{array}{lll}\sin \alpha \cos \beta & \sin \alpha \sin \beta & \cos \alpha\end{array}\right]$, the ellipticity indicator $q$ is defined as $q=\min \left(q_{1}, q_{2}, q_{3}\right)$, with $\left\{q_{1}, q_{2}, q_{3}\right\}$ the leading minors of $\mathcal{C}_{\boldsymbol{V} V}$. Negative values of $q$ are associated with loss of ellipticity. Tables 12 and 13 show the distribution of $q$ for different snapshots of the cardiac cycle for Simulation-I (active stress) and Simulation-III (active strain) when considering or when disregarding the Macaulay brackets at a given Gauss point of the idealised set of ventricles in 19. It can be observed that no loss of ellipticity was obtained at the given Gauss point when the Macaulay brackets are activated. However, when these are disabled, loss of ellipticity can be observed for both active stress and active strain approaches. Notice that negative values of $q$ have been truncated to zero in both figures.

Tables 14 and 15 show the contour plot of the ellipticity indicator $q$ over the entire domain when the Macaulay brackets in Holzapfel-Ogden model (18) are not considered for: (a) different snapshots of the cardiac circle and for (b) active stress and active strain approaches. In these figures, loss of ellipticity is associated with orange regions. Furthermore, both figures include the contour plot of an alternative instability indicator associated with loss of polyconvexity. Specifically, red areas in these figures are associated with the loss of positive definiteness of the Hessian operator $\mathbb{H}_{W}$ (12). Although polyconvexity and ellipticity are not equivalent concepts, an extremely good agreement between loss of ellipticity and loss of polyconvexity is obtained for the constitutive model considered. When considering the Macaulay brackets, loss of ellipticity was rarely detected. 
Table 14

Numerical example 4.3. Simulation details: activation type: active stress; ionic model: Bueno-Orovio; activation law: Nash \& Panfilov; mixed formulation: ELEM1-MFA- $T_{a}$. Snapshots for time $t=\{20,40,80,150,250,290\}$ (ms) of the cardiac cycle. Loss of ellipticity (orange regions) and loss of polyconvexity (red regions) when the Macaulay brackets are not considered in Holzapfel-Ogden model (18).
Ellipticity
Polyconvexity
Ellipticity
Polyconvexity

$20 \mathrm{~ms}$
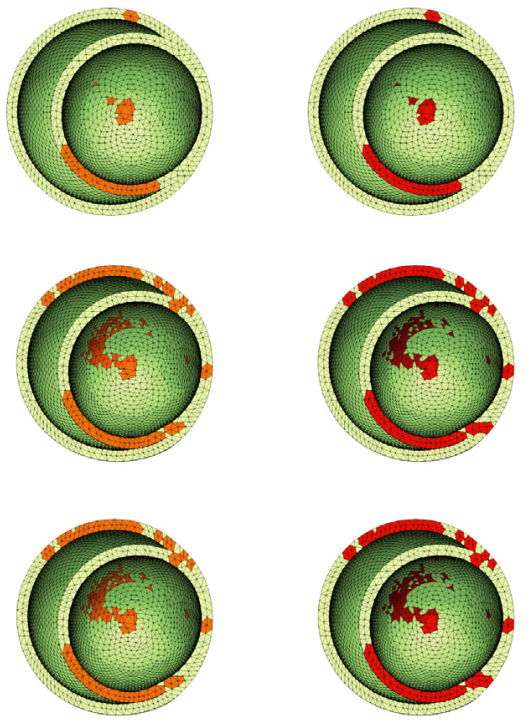

$150 \mathrm{~ms}$
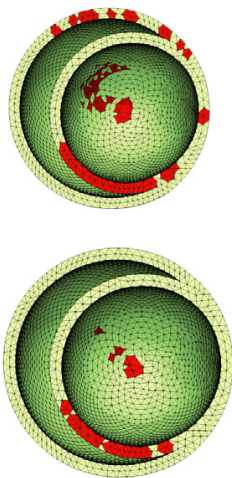

$290 \mathrm{~ms}$
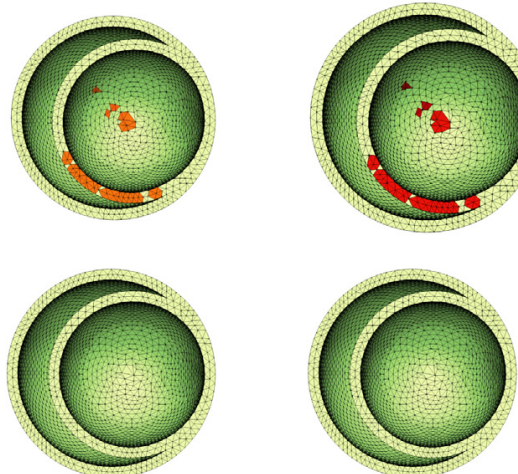

\section{Table 15}

Numerical example 4.3. Simulation details: activation type: active strain; ionic model: Bueno-Orovio; activation law: Rossi et al.; mixed formulation: ELEM1-MFA- $T_{a}$. Snapshots for time $t=\{10,20,40,80,150,250,290,320\}$ (ms) of the cardiac cycle. Loss of ellipticity (orange regions) and loss of polyconvexity (red regions) when the Macaulay brackets are not considered in Holzapfel-Ogden model (18).
Ellipticity
Polyconvexity
Ellipticity
Polyconvexity

$20 \mathrm{~ms}$
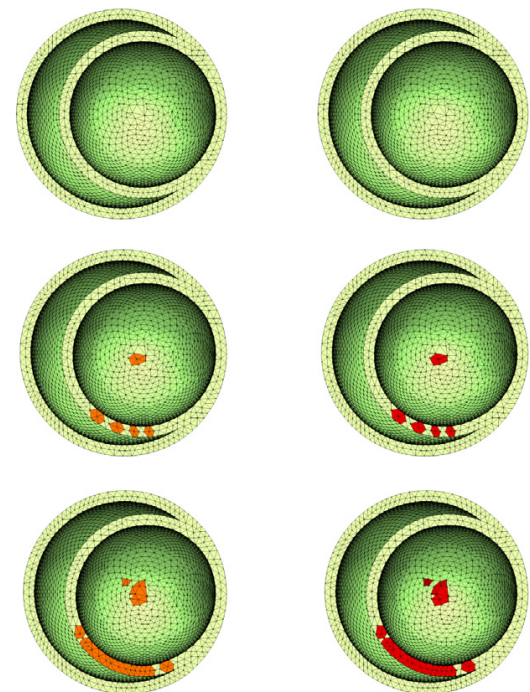

$110 \mathrm{~ms}$

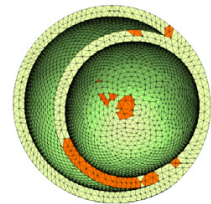

$250 \mathrm{~ms}$
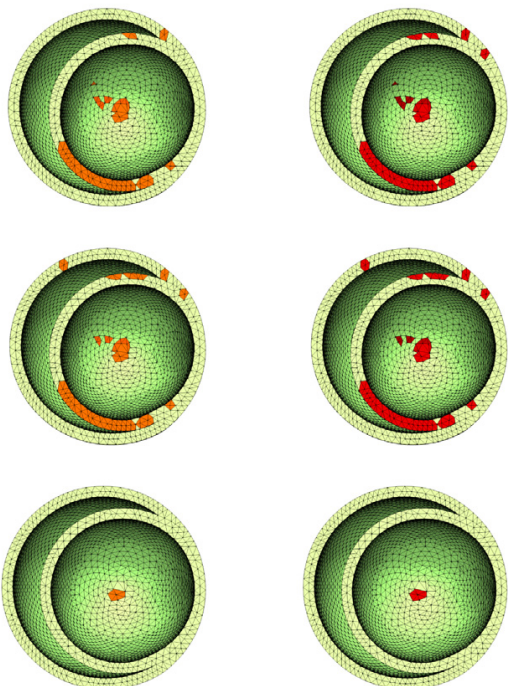

$290 \mathrm{~ms}$

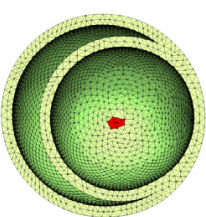

This study is in good agreement with the derivations in Appendix A. Specifically, it demonstrates numerically that the strain energy in (18) is very prone to exhibit loss of ellipticity and hence, polyconvexity when the Macauly brackets are not considered in both active stress and active strain approaches. 


\section{Concluding remarks}

This paper presents a novel computational framework for the numerical simulation of the electromechanical response of the myocardium during the cardiac cycle. Two new mixed formulations, tailor-made for active stress and active strain coupling approaches, have been developed and used in conjunction with two different ionic models, namely Bueno-Orovio [49] and Ten Tusccher [47,48]. Taking as a reference the mixed formulations introduced by Bonet et al. [30] in the context of nonlinear elasticity, the proposed formulations include as unknown fields the geometry and the transmembrane potential (and possibly a Lagrange multiplier enforcing weakly the incompressibility constraint) as well as the deformation gradient tensor, its cofactor and its determinant, the gradient of the transmembrane potential and their respective work conjugates. The Finite Element implementation of these formulations is shown in this paper. Crucially, the superiority of these formulations with respect to classical low order Finite Element implementations is shown in this paper. A comprehensive and rigorous study of different ionic models (i.e Bueno-Orovio and Ten Tusscher) and electromechanical activation couplings (i.e active strain and active stress) has been carried out. An analytical and numerical analysis of the possible loss of ellipticity and polyconvexity of one of the most widely used constitutive models in the context of cardiac mechanics is carried out in this paper, putting forward possible polyconvexifications of the existing model. (4) In addition, an invariant representation of Guccione's constitutive model is proposed. Finally, a series of numerical examples are included in order to demonstrate the applicability and robustness of the proposed formulations.

The computational framework developed in this manuscript will be used in a follow up publication with the aim of carrying out a thorough investigation of different solution techniques for the coupled electromechanical problem. Specifically, the accuracy and computational efficiency of staggered schemes will be analysed and compared to those of the monolithical approach advocated for in this paper. The conclusions of this forthcoming study could be very beneficial for the analysis of more complex and computationally demanding simulations such as those involving the simulation of cardiac arrhythmias.

\section{Acknowledgements}

The second and third authors acknowledge the support provided by the Sêr Cymru National Research Network under the Sêr Cymru II Fellowship "Virtual engineering of the new generation of biomimetic artificial muscles", funded by the European Regional Development Fund. The third author acknowledges the financial support received through the European Training Network AdMoRe (Project ID: 675919).

\section{Appendix A. Ellipticity (rank-one convexity) of Holzapfel-Ogden model for passive response of myocardium}

In this Section we study the possible loss of ellipticity of the constitutive model in Eq. (18). For this, we introduce an additive decomposition of the ellipticity indicator $\mathcal{I}_{W}$ in (15) as

$$
\mathcal{I}_{\mathcal{W}}=\mathcal{I}_{\hat{W}}+\mathcal{I}_{U}+\mathcal{I}_{W_{f_{0}}^{\text {ani }}}+\mathcal{I}_{W_{s_{0}}^{\text {ani }}}+\mathcal{I}_{W_{f_{0} s_{0}}^{\text {ani }}}
$$

with each term defined as

$$
\begin{aligned}
\mathcal{I}_{\hat{W}} & =D^{2} \hat{W}[\boldsymbol{u} \otimes \boldsymbol{V} ; \boldsymbol{u} \otimes \boldsymbol{V}] ; \\
\mathcal{I}_{U} & =D^{2} U[\boldsymbol{u} \otimes \boldsymbol{V} ; \boldsymbol{u} \otimes \boldsymbol{V}] ; \\
\mathcal{I}_{W_{f_{0}}^{\text {ani }}} & =D^{2}\left(\frac{a_{f}}{2 b_{f}}\left(e^{b_{f}\left\langle I_{4, f_{0}}-1\right\rangle^{2}}-1\right)\right)[\boldsymbol{u} \otimes \boldsymbol{V} ; \boldsymbol{u} \otimes \boldsymbol{V}] ; \\
\mathcal{I}_{W_{s_{0}}^{\text {ani }}} & =D^{2}\left(\frac{a_{s}}{2 b_{s}}\left(e^{b_{s}\left\langle I_{4, s_{0}}-1\right\rangle^{2}}-1\right)\right)[\boldsymbol{u} \otimes \boldsymbol{V} ; \boldsymbol{u} \otimes \boldsymbol{V}] ; \\
\mathcal{I}_{W_{f_{0}}^{\text {ani }} s_{0}} & =D^{2}\left(\frac{a_{f s}}{2 b_{f s}}\left(e^{b_{f s} I_{8, f_{0}, s_{0}}^{2}}-1\right)\right)[\boldsymbol{u} \otimes \boldsymbol{V} ; \boldsymbol{u} \otimes \boldsymbol{V}] .
\end{aligned}
$$

The ellipticity indicator for the isochoric isotropic term can be expressed as

$$
\begin{aligned}
\mathcal{I}_{\hat{W}} & =a b e^{b\left(I I_{\hat{\boldsymbol{F}}_{\boldsymbol{x}}}-3\right)} J_{\boldsymbol{x}}^{-10 / 3} \alpha^{2}+a e^{b\left(I I_{\hat{\boldsymbol{F}}_{\boldsymbol{x}}}-3\right)} J_{\boldsymbol{x}}^{-8 / 3} I I_{\boldsymbol{A}}+\frac{a}{5} e^{b\left(I_{\hat{\boldsymbol{F}}_{\boldsymbol{x}}}-3\right)} J_{\boldsymbol{x}}^{-2 / 3}(\boldsymbol{u} \cdot \boldsymbol{u})(\boldsymbol{V} \cdot \boldsymbol{V}) \\
& \geq \frac{a}{5} e^{b\left(I I_{\hat{\boldsymbol{F}}_{\boldsymbol{x}}}-3\right)} J_{\boldsymbol{x}}^{-2 / 3}(\boldsymbol{u} \cdot \boldsymbol{u})(\boldsymbol{V} \cdot \boldsymbol{V}) \geq 0 .
\end{aligned}
$$


with

$$
\alpha=\sqrt{2} J_{\boldsymbol{x}}\left(\boldsymbol{u} \cdot \boldsymbol{F}_{\boldsymbol{x}} \boldsymbol{V}\right)-\frac{\sqrt{2}}{3}\left(\boldsymbol{u} \cdot \boldsymbol{H}_{\boldsymbol{x}} \boldsymbol{V}\right) I I_{\boldsymbol{F}_{\boldsymbol{x}}} ; \quad \boldsymbol{A}=\frac{2}{\sqrt{5}} J_{\boldsymbol{x}}(\boldsymbol{u} \otimes \boldsymbol{V})-\frac{\sqrt{5}}{3}\left(\boldsymbol{u} \cdot \boldsymbol{H}_{\boldsymbol{x}} \boldsymbol{V}\right) \boldsymbol{F}_{\boldsymbol{x}}
$$

For the volumetric contribution $U\left(J_{x}\right), \mathcal{I}_{U}$ is obtained as

$$
\mathcal{I}(U)=\kappa\left(\boldsymbol{u} \cdot \boldsymbol{H}_{\boldsymbol{x}} \boldsymbol{V}\right)^{2} \geq 0 .
$$

The anisotropic contribution $\mathcal{I}_{W_{f_{0}}^{\text {ani }}}$ can be obtained as

$$
\begin{aligned}
\mathcal{I}_{W_{f_{0}}^{\mathrm{ani}}} & =4\left(2 b_{f}\left\langle I_{4, f_{0}}-1\right\rangle^{2}+1\right) a_{f} e^{b_{f}\left\langle I_{4, f_{0}}-1\right\rangle^{2}}\left(\boldsymbol{u} \cdot \boldsymbol{F}_{\boldsymbol{x}} \boldsymbol{f}_{0}\right)^{2}\left(\boldsymbol{V} \cdot \boldsymbol{f}_{0}\right)^{2} \\
& +2 a_{f} e^{b_{f}\left(I_{4, f_{0}}-1\right\rangle^{2}}\left\langle I_{4, f_{0}}-1\right\rangle(\boldsymbol{u} \cdot \boldsymbol{u})\left(\boldsymbol{V} \cdot \boldsymbol{f}_{0}\right)^{2} \geq 0 .
\end{aligned}
$$

Similarly, the anisotropic contribution $\mathcal{I}_{W_{s_{0}}}$ an can be obtained as

$$
\begin{aligned}
\mathcal{I}_{W_{s_{0}}^{\text {ani }}} & =4\left(2 b_{s}\left\langle I_{4, s_{0}}-1\right\rangle^{2}+1\right) a_{s} e^{b_{s}\left(I_{4, s_{0}}-1\right\rangle^{2}}\left(\boldsymbol{u} \cdot \boldsymbol{F}_{\boldsymbol{x}} \boldsymbol{s}_{0}\right)^{2}\left(\boldsymbol{V} \cdot \boldsymbol{s}_{0}\right)^{2} \\
& +2 a_{s} e^{b_{s}\left(I_{4, s_{0}}-1\right\rangle^{2}}\left\langle I_{4, s_{0}}-1\right\rangle(\boldsymbol{u} \cdot \boldsymbol{u})\left(\boldsymbol{V} \cdot \boldsymbol{s}_{0}\right)^{2} \geq 0 .
\end{aligned}
$$

Finally, the anisotropic contribution $\mathcal{I}_{W_{f_{0}}^{\text {ani }} s_{0}}$ can be obtained as

$$
\begin{aligned}
& \mathcal{I}_{W_{f_{0} s_{0}}^{\mathrm{ani}}}=\left(2 b_{f s} I_{8, f_{0} s_{0}}^{2}+1\right) a_{f s} e^{b_{f s} I_{4, f_{0} s_{0}}^{2}}\left(\left(\boldsymbol{u} \cdot \boldsymbol{F}_{\boldsymbol{x}} \boldsymbol{f}_{0}\right)\left(\boldsymbol{V} \cdot \boldsymbol{s}_{0}\right)+\left(\boldsymbol{u} \cdot \boldsymbol{F}_{\boldsymbol{x}} \boldsymbol{s}_{0}\right)\left(\boldsymbol{V} \cdot \boldsymbol{f}_{0}\right)\right)^{2} \\
& +2 a_{f s} e^{b_{f s} I_{8, f_{0} s_{0}}^{2}} I_{8, f_{0} s_{0}}(\boldsymbol{u} \cdot \boldsymbol{u})\left(\boldsymbol{V} \cdot \boldsymbol{f}_{0}\right)\left(\boldsymbol{V} \cdot \boldsymbol{s}_{0}\right)
\end{aligned}
$$

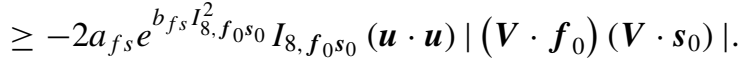

As a result, given the unconditional positiveness of $\mathcal{I}_{\hat{W}}, \mathcal{I}_{U}, \mathcal{I}_{W_{f_{0}}^{\text {ani }}}$ and $\mathcal{I}_{W_{s_{0}}}$, the total ellipticity indicator $\mathcal{I}_{W}$ can be written as

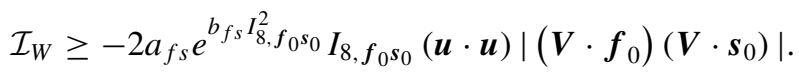

Alternatively, if the Macauly brackets are not considered, the anisotropic contributions $\mathcal{I}_{W_{f_{0}}^{\text {ani }}}$ and $\mathcal{I}_{W_{s_{0}}}$ ani

$$
\begin{aligned}
& \mathcal{I}_{W_{f_{0}}^{\mathrm{ani}}}=4\left(2 b_{f}\left(I_{4, f_{0}}-1\right)^{2}+1\right) a_{f} e^{b_{f}\left(I_{4, f_{0}}-1\right)^{2}}\left(\boldsymbol{u} \cdot \boldsymbol{F}_{\boldsymbol{x}} \boldsymbol{f}_{0}\right)^{2}\left(\boldsymbol{V} \cdot \boldsymbol{f}_{0}\right)^{2} \\
&+2 a_{f} e^{b_{f}\left(I_{4, f_{0}}-1\right)^{2}}\left(I_{4, f_{0}}-1\right)(\boldsymbol{u} \cdot \boldsymbol{u})\left(\boldsymbol{V} \cdot \boldsymbol{f}_{0}\right)^{2} \\
& \geq-2 a_{f} e^{b_{f}\left(I_{4, f_{0}}-1\right)^{2}}\left|\left(I_{4, f_{0}}-1\right)\right|(\boldsymbol{u} \cdot \boldsymbol{u})\left(\boldsymbol{V} \cdot \boldsymbol{f}_{0}\right)^{2} \\
& \mathcal{I}_{W_{s_{0}}^{\mathrm{ani}}}=4\left(2 b_{s}\left(I_{4, s_{0}}-1\right)^{2}+1\right) a_{s} e^{b_{s}\left(I_{4, s_{0}}-1\right)^{2}}\left(\boldsymbol{u} \cdot \boldsymbol{F}_{\boldsymbol{x}} \boldsymbol{s}_{0}\right)^{2}\left(\boldsymbol{V} \cdot \boldsymbol{s}_{0}\right)^{2} \\
&+2 a_{s} e^{b_{f}\left(I_{4, s_{0}}-1\right)^{2}}\left(I_{4, s_{0}}-1\right)(\boldsymbol{u} \cdot \boldsymbol{u})\left(\boldsymbol{V} \cdot \boldsymbol{s}_{0}\right)^{2} \\
& \geq-2 a_{s} e^{b_{s}\left(I_{4, s_{0}}-1\right)^{2}}\left|\left(I_{4, s_{0}}-1\right)\right|(\boldsymbol{u} \cdot \boldsymbol{u})\left(\boldsymbol{V} \cdot \boldsymbol{s}_{0}\right)^{2} .
\end{aligned}
$$

The total contribution $\mathcal{I}_{W}$ when not considering the Macauly brackets is

$$
\begin{aligned}
\mathcal{I}_{W} & =-2 a_{f s} e^{b_{f s} I_{8, f_{0} s_{0}}^{2}} I_{8, f_{0} s_{0}}(\boldsymbol{u} \cdot \boldsymbol{u})\left|\left(\boldsymbol{V} \cdot \boldsymbol{f}_{0}\right)\left(\boldsymbol{V} \cdot \boldsymbol{s}_{0}\right)\right| . \\
& -2 a_{f} e^{b_{s}\left(I_{4, f_{0}}-1\right)^{2}}\left|\left(I_{4, f_{0}}-1\right)\right|(\boldsymbol{u} \cdot \boldsymbol{u})\left(\boldsymbol{V} \cdot \boldsymbol{f}_{0}\right)^{2} \\
& -2 a_{s} e^{b_{s}\left(I_{4, s_{0}}-1\right)^{2}}\left|\left(I_{4, s_{0}}-1\right)\right|(\boldsymbol{u} \cdot \boldsymbol{u})\left(\boldsymbol{V} \cdot \boldsymbol{s}_{0}\right)^{2} .
\end{aligned}
$$

The larger number of terms in (B.21) as opposed to (B.18) leads therefore in general to a model which is more prone for loss of ellipticity, specially when fibres $\boldsymbol{f}_{0}$ and $\boldsymbol{s}_{0}$ are contracted, namely $I_{4, f_{0}}<0$ and $I_{4, s_{0}}<0$. 


\section{Appendix B. Invariant-based representation for Guccione's law}

Guccione et al. modelled the heart tissue as a three-dimensional transverse isotropic material in [79] using a Fungtype exponential energy law [80] as

$$
\Psi\left(\boldsymbol{E}^{\star}\right)=\frac{K}{2}\left(e^{Q\left(\boldsymbol{E}^{\star}\right)}-1\right)
$$

where $\boldsymbol{E}^{*}$ represents the local Green-Lagrange strain tensor, expressed with respect to the local basis $\left\{\boldsymbol{f}_{0}, \boldsymbol{s}_{0}, \boldsymbol{n}_{0}\right\}$. Let $\boldsymbol{R}$ be the rotation matrix from the local basis to the global system of coordinates. $\boldsymbol{E}^{\star}$ can be related to its global counterpart $\boldsymbol{E}=\frac{1}{2}\left(\boldsymbol{F}_{\boldsymbol{x}}^{T} \boldsymbol{F}_{\boldsymbol{x}}-\boldsymbol{I}\right)$ as $\boldsymbol{E}^{\star}=\boldsymbol{R}^{T} \boldsymbol{E} \boldsymbol{R}$. The exponent $Q\left(\boldsymbol{E}^{\star}\right)$ in (B.1) is defined in terms of the local Green-Lagrange strain tensor as

$$
Q\left(\boldsymbol{E}^{\star}\right)=c_{f}{E^{\star 2}}_{11}+c_{t}\left(E_{22}^{\star 2}+E_{33}^{\star 2}+E_{23}^{\star 2}+E_{32}^{\star 2}\right)+c_{f s}\left(E_{12}^{\star 2}+E_{21}^{\star 2}+E_{13}^{\star 2}+E_{31}^{\star 2}\right),
$$

where $\left\{K, c_{f}, c_{t}, c_{f s}\right\}$ represent material parameters. Let $\left\{\boldsymbol{e}_{1}, \boldsymbol{e}_{2}, \boldsymbol{e}_{3}\right\}$ be

$$
\left[\begin{array}{lll}
1 & 0 & 0
\end{array}\right]^{T} ; \quad\left[\begin{array}{ccc}
0 & 1 & 0
\end{array}\right]^{T} ; \quad\left[\begin{array}{lll}
0 & 0 & 1
\end{array}\right]^{T} .
$$

Then $Q\left(\boldsymbol{E}^{\star}\right)$ can be expressed in terms of $\left\{\left(\boldsymbol{e}_{1} \cdot \boldsymbol{E}^{\star} \boldsymbol{e}_{1}\right)^{2}, \boldsymbol{E}^{\star} \boldsymbol{e}_{1} \cdot \boldsymbol{E}^{\star} \boldsymbol{e}_{1}, \boldsymbol{E}^{\star}: \boldsymbol{E}^{\star}\right\}$, as

$$
Q\left(\boldsymbol{E}^{*}\right)=4 A\left(\boldsymbol{e}_{1} \cdot \boldsymbol{E}^{*} \boldsymbol{e}_{1}\right)^{2}+4 B\left(\boldsymbol{E}^{*} \boldsymbol{e}_{1} \cdot \boldsymbol{E}^{*} \boldsymbol{e}_{1}\right)+4 C\left(\boldsymbol{E}^{*}: \boldsymbol{E}^{*}\right),
$$

with

$$
A=\frac{c_{f}-2 c_{f s}+c_{t}}{2} ; \quad B=\frac{c_{f s}-c_{t}}{4} ; \quad C=\frac{c_{f}}{4} .
$$

Notice that the three invariants $\left\{\left(\boldsymbol{e}_{1} \cdot \boldsymbol{E}^{\star} \boldsymbol{e}_{1}\right)^{2}, \boldsymbol{E}^{\star} \boldsymbol{e}_{1} \cdot \boldsymbol{E}^{\star} \boldsymbol{e}_{1}, \boldsymbol{E}^{\star}: \boldsymbol{E}^{\star}\right\}$ can be equivalently expressed with respect to the global system of coordinates as

$$
\boldsymbol{e}_{1} \cdot \boldsymbol{E}^{\star} \boldsymbol{e}_{1}=\boldsymbol{f}_{0} \cdot \boldsymbol{E} \boldsymbol{f}_{0} ; \quad \boldsymbol{E}^{*}: \boldsymbol{E}^{*}=\boldsymbol{E}: \boldsymbol{E} ; \quad \boldsymbol{E}^{*} \boldsymbol{e}_{1} \cdot \boldsymbol{E}^{*} \boldsymbol{e}_{1}=\boldsymbol{E} \boldsymbol{f}_{0} \cdot \boldsymbol{E} \boldsymbol{f}_{0},
$$

and hence, an equivalent expression for $Q$ can be obtained in terms of the global Green-Lagrange strain tensor $\boldsymbol{E}$ as

$$
Q(\boldsymbol{E})=4 A\left(\boldsymbol{f}_{0} \cdot \boldsymbol{E} \boldsymbol{f}_{0}\right)^{2}+4 B\left(\boldsymbol{E} \boldsymbol{f}_{0} \cdot \boldsymbol{E} \boldsymbol{f}_{0}\right)+4 C(\boldsymbol{E}: \boldsymbol{E}),
$$

This (objective) invariant representation of Guccione's model is now very well suited for numerical simulation via Finite Element implementation. Alternatively, it is possible to express the invariants $\left\{\left(\boldsymbol{f}_{0} \cdot \boldsymbol{E} \boldsymbol{f}_{0}\right)^{2}, \boldsymbol{E} \boldsymbol{f}_{0} \cdot \boldsymbol{E} \boldsymbol{f}_{0}, \boldsymbol{E}: \boldsymbol{E}\right\}$ in terms of the kinematic strain measures $\left\{\boldsymbol{F}_{\boldsymbol{x}}, \boldsymbol{H}_{\boldsymbol{x}}, J_{\boldsymbol{x}}\right\}$ as

$$
\begin{aligned}
\boldsymbol{f}_{0} \cdot \boldsymbol{E} \boldsymbol{f}_{0} & =\frac{I_{4, f_{0}}-1}{2} ; \\
\boldsymbol{E} \boldsymbol{f}_{0} \cdot \boldsymbol{E} \boldsymbol{f}_{0} & =\frac{I I_{\boldsymbol{F}_{\boldsymbol{x}}} I_{4, \boldsymbol{f}_{0}}-I I_{\boldsymbol{H}_{\boldsymbol{x}}}+I_{4, \boldsymbol{H}_{\boldsymbol{x}}, \boldsymbol{f}_{0}}-2 I_{4, \boldsymbol{f}_{0}}+1}{4} ; \quad I_{4, \boldsymbol{H}_{\boldsymbol{x}}, \boldsymbol{f}_{0}}=\boldsymbol{H}_{\boldsymbol{x}} \boldsymbol{f}_{0} \cdot \boldsymbol{H}_{\boldsymbol{x}} \boldsymbol{f}_{0} ; \\
\boldsymbol{E}: \boldsymbol{E} & =\frac{I I_{\boldsymbol{F}_{\boldsymbol{x}}}^{2}-2 I I_{\boldsymbol{H}_{\boldsymbol{x}}}-2 I I_{\boldsymbol{F}_{\boldsymbol{x}}}+3}{4} .
\end{aligned}
$$

Making use of (B.8), $Q\left(\boldsymbol{E}^{\star}\right)$ can be equivalently expressed as $Q(\boldsymbol{E})=\widetilde{Q}\left(\boldsymbol{F}_{\boldsymbol{x}}, \boldsymbol{H}_{\boldsymbol{x}}, J_{\boldsymbol{x}}\right)$, with

$$
\widetilde{Q}\left(\boldsymbol{F}_{\boldsymbol{x}}, \boldsymbol{H}_{\boldsymbol{x}}, J_{\boldsymbol{x}}\right)=A Q_{\text {aniso }, 1}\left(\boldsymbol{F}_{\boldsymbol{x}}\right)+B Q_{\text {aniso }, 2}\left(\boldsymbol{F}_{\boldsymbol{x}}, \boldsymbol{H}_{\boldsymbol{x}}\right)+C Q_{\text {iso }}\left(\boldsymbol{F}_{\boldsymbol{x}}, \boldsymbol{H}_{\boldsymbol{x}}, J_{\boldsymbol{x}}\right),
$$

where

$$
\begin{aligned}
Q_{\text {aniso, }, 1}\left(\boldsymbol{F}_{\boldsymbol{x}}\right) & =\left(I_{4, f_{0}}-1\right)^{2} ; \\
Q_{\text {aniso }, 2}\left(\boldsymbol{F}_{\boldsymbol{x}}, \boldsymbol{H}_{\boldsymbol{x}}\right) & =I I_{\boldsymbol{F}_{\boldsymbol{x}}} I_{4, f_{0}}-I I_{\boldsymbol{H}_{\boldsymbol{x}}}+I_{4, \boldsymbol{H}_{\boldsymbol{x}}, f_{0}}-2 I_{4, f_{0}}+1 ; \\
Q_{\text {iso }}\left(\boldsymbol{F}_{\boldsymbol{x}}, \boldsymbol{H}_{\boldsymbol{x}}, J_{\boldsymbol{x}}\right) & =I I_{\boldsymbol{F}_{\boldsymbol{x}}}^{2}-2 I I_{\boldsymbol{H}_{\boldsymbol{x}}}-2 I I_{\boldsymbol{F}_{\boldsymbol{x}}}+3 .
\end{aligned}
$$

We study the possible loss of ellipticity of the constitutive model in Eq. (18). For this, we introduce an additive decomposition of the ellipticity indicator $\mathcal{I}_{W}$ in (15) as

$$
\mathcal{I}_{\mathcal{W}}=\mathcal{I}_{\hat{W}}+\mathcal{I}_{U}+\mathcal{I}_{W_{f_{0}}^{\text {ani }}}+\mathcal{I}_{W_{s_{0}}^{\text {ani }}}+\mathcal{I}_{W_{f_{0} s_{0}}^{\text {ani }}}
$$


with each term defined as

$$
\begin{aligned}
\mathcal{I}_{\hat{W}} & =D^{2} \hat{W}[\boldsymbol{u} \otimes \boldsymbol{V} ; \boldsymbol{u} \otimes \boldsymbol{V}] ; \\
\mathcal{I}_{U} & =D^{2} U[\boldsymbol{u} \otimes \boldsymbol{V} ; \boldsymbol{u} \otimes \boldsymbol{V}] ; \\
\mathcal{I}_{W_{f_{0}}^{\text {ani }}} & =D^{2}\left(\frac{a_{f}}{2 b_{f}}\left(e^{b_{f}\left\langle I_{4, f_{0}}-1\right\rangle^{2}}-1\right)\right)[\boldsymbol{u} \otimes \boldsymbol{V} ; \boldsymbol{u} \otimes \boldsymbol{V}] ; \\
\mathcal{I}_{W_{s_{0}}^{\text {ani }}} & =D^{2}\left(\frac{a_{s}}{2 b_{s}}\left(e^{b_{s}\left\langle I_{4, s_{0}}-1\right\rangle^{2}}-1\right)\right)[\boldsymbol{u} \otimes \boldsymbol{V} ; \boldsymbol{u} \otimes \boldsymbol{V}] ; \\
\mathcal{I}_{W_{f_{0}}^{\text {ani }} s_{0}} & =D^{2}\left(\frac{a_{f s}}{2 b_{f s}}\left(e^{b_{f s} I_{8, f_{0}, s_{0}}^{2}}-1\right)\right)[\boldsymbol{u} \otimes \boldsymbol{V} ; \boldsymbol{u} \otimes \boldsymbol{V}] .
\end{aligned}
$$

The ellipticity indicator for the isochoric isotropic term can be expressed as

$$
\begin{aligned}
\mathcal{I}_{\hat{W}} & =a b e^{b\left(I I_{\hat{\boldsymbol{F}}_{\boldsymbol{x}}}-3\right)} J_{\boldsymbol{x}}^{-10 / 3} \alpha^{2}+a e^{b\left(I I_{\hat{\boldsymbol{F}}_{\boldsymbol{x}}}-3\right)} J_{\boldsymbol{x}}^{-8 / 3} I I_{\boldsymbol{A}}+\frac{a}{5} e^{b\left(I I_{\hat{\boldsymbol{F}}_{\boldsymbol{x}}}-3\right)} J_{\boldsymbol{x}}^{-2 / 3}(\boldsymbol{u} \cdot \boldsymbol{u})(\boldsymbol{V} \cdot \boldsymbol{V}) \\
& \geq \frac{a}{5} e^{b\left(I_{\hat{\boldsymbol{F}}_{\boldsymbol{x}}}-3\right)} J_{\boldsymbol{x}}^{-2 / 3}(\boldsymbol{u} \cdot \boldsymbol{u})(\boldsymbol{V} \cdot \boldsymbol{V}) \geq 0 .
\end{aligned}
$$

with

$$
\alpha=\sqrt{2} J_{\boldsymbol{x}}\left(\boldsymbol{u} \cdot \boldsymbol{F}_{\boldsymbol{x}} \boldsymbol{V}\right)-\frac{\sqrt{2}}{3}\left(\boldsymbol{u} \cdot \boldsymbol{H}_{\boldsymbol{x}} \boldsymbol{V}\right) I I_{\boldsymbol{F}_{\boldsymbol{x}}} ; \quad \boldsymbol{A}=\frac{2}{\sqrt{5}} J_{\boldsymbol{x}}(\boldsymbol{u} \otimes \boldsymbol{V})-\frac{\sqrt{5}}{3}\left(\boldsymbol{u} \cdot \boldsymbol{H}_{\boldsymbol{x}} \boldsymbol{V}\right) \boldsymbol{F}_{\boldsymbol{x}}
$$

For the volumetric contribution $U\left(J_{x}\right), \mathcal{I}_{U}$ is obtained as

$$
\mathcal{I}(U)=\kappa\left(\boldsymbol{u} \cdot \boldsymbol{H}_{\boldsymbol{x}} \boldsymbol{V}\right)^{2} \geq 0 .
$$

The anisotropic contribution $\mathcal{I}_{W_{f_{0}}^{\text {ani }}}$ can be obtained as

$$
\begin{aligned}
\mathcal{I}_{W_{f_{0}}^{\mathrm{ani}}} & =4\left(2 b_{f}\left\langle I_{4, f_{0}}-1\right\rangle^{2}+1\right) a_{f} e^{b_{f}\left\langle I_{4, f_{0}}-1\right\rangle^{2}}\left(\boldsymbol{u} \cdot \boldsymbol{F}_{\boldsymbol{x}} \boldsymbol{f}_{0}\right)^{2}\left(\boldsymbol{V} \cdot \boldsymbol{f}_{0}\right)^{2} \\
& +2 a_{f} e^{b_{f}\left(I_{4, f_{0}}-1\right\rangle^{2}}\left\langle I_{4, f_{0}}-1\right\rangle(\boldsymbol{u} \cdot \boldsymbol{u})\left(\boldsymbol{V} \cdot \boldsymbol{f}_{0}\right)^{2} \geq 0 .
\end{aligned}
$$

Similarly, the anisotropic contribution $\mathcal{I}_{W_{s_{0}}}$ an can be obtained as

$$
\begin{aligned}
\mathcal{I}_{W_{s_{0}}^{\text {ani }}} & =4\left(2 b_{s}\left\langle I_{4, s_{0}}-1\right\rangle^{2}+1\right) a_{s} e^{b_{s}\left(I_{4, s_{0}}-1\right\rangle^{2}}\left(\boldsymbol{u} \cdot \boldsymbol{F}_{\boldsymbol{x}} \boldsymbol{s}_{0}\right)^{2}\left(\boldsymbol{V} \cdot \boldsymbol{s}_{0}\right)^{2} \\
& +2 a_{s} e^{b_{s}\left(I_{4, s_{0}}-1\right)^{2}}\left\langle I_{4, s_{0}}-1\right\rangle(\boldsymbol{u} \cdot \boldsymbol{u})\left(\boldsymbol{V} \cdot \boldsymbol{s}_{0}\right)^{2} \geq 0 .
\end{aligned}
$$

Finally, the anisotropic contribution $\mathcal{I}_{W_{f_{0}}^{\text {ani }} s_{0}}$ can be obtained as

$$
\begin{aligned}
& \mathcal{I}_{W_{f_{0} s_{0}}^{\mathrm{ani}}}=\left(2 b_{f s} I_{8, f_{0} s_{0}}^{2}+1\right) a_{f s} e^{b_{f s} I_{4, f_{0}}^{2} s_{0}}\left(\left(\boldsymbol{u} \cdot \boldsymbol{F}_{\boldsymbol{x}} \boldsymbol{f}_{0}\right)\left(\boldsymbol{V} \cdot \boldsymbol{s}_{0}\right)+\left(\boldsymbol{u} \cdot \boldsymbol{F}_{\boldsymbol{x}} \boldsymbol{s}_{0}\right)\left(\boldsymbol{V} \cdot \boldsymbol{f}_{0}\right)\right)^{2} \\
& +2 a_{f s} e^{b_{f s} I_{8, f_{0} s_{0}}^{2}} I_{8, f_{0} s_{0}}(\boldsymbol{u} \cdot \boldsymbol{u})\left(\boldsymbol{V} \cdot \boldsymbol{f}_{0}\right)\left(\boldsymbol{V} \cdot \boldsymbol{s}_{0}\right)
\end{aligned}
$$

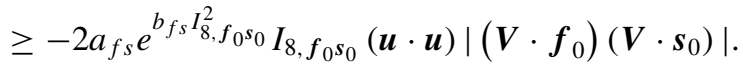

As a result, given the unconditional positiveness of $\mathcal{I}_{\hat{W}}, \mathcal{I}_{U}, \mathcal{I}_{W_{f_{0}}^{\text {ani }}}$ and $\mathcal{I}_{W_{s_{0}}^{\text {ani }}}$, the total ellipticity indicator $\mathcal{I}_{W}$ can be written as

$$
\mathcal{I}_{W} \geq-2 a_{f s} e^{b_{f s} I_{8, f_{0} s_{0}}^{2}} I_{8, f_{0} s_{0}}(\boldsymbol{u} \cdot \boldsymbol{u})\left|\left(\boldsymbol{V} \cdot \boldsymbol{f}_{0}\right)\left(\boldsymbol{V} \cdot \boldsymbol{s}_{0}\right)\right| .
$$


Alternatively, if the Macauly brackets are not considered, the anisotropic contributions $\mathcal{I}_{W_{f_{0}}^{\text {ani }}}$ and $\mathcal{I}_{W_{s_{0}} \text { ani }}$ are

$$
\begin{aligned}
& \mathcal{I}_{W_{f_{0}}^{\mathrm{ani}}}=4\left(2 b_{f}\left(I_{4, f_{0}}-1\right)^{2}+1\right) a_{f} e^{b_{f}\left(I_{4, f_{0}}-1\right)^{2}}\left(\boldsymbol{u} \cdot \boldsymbol{F}_{\boldsymbol{x}} \boldsymbol{f}_{0}\right)^{2}\left(\boldsymbol{V} \cdot \boldsymbol{f}_{0}\right)^{2} \\
&+2 a_{f} e^{b_{f}\left(I_{4, f_{0}}-1\right)^{2}}\left(I_{4, f_{0}}-1\right)(\boldsymbol{u} \cdot \boldsymbol{u})\left(\boldsymbol{V} \cdot \boldsymbol{f}_{0}\right)^{2} \\
& \geq-2 a_{f} e^{b_{f}\left(I_{4, f_{0}}-1\right)^{2}}\left|\left(I_{4, f_{0}}-1\right)\right|(\boldsymbol{u} \cdot \boldsymbol{u})\left(\boldsymbol{V} \cdot \boldsymbol{f}_{0}\right)^{2} \\
& \mathcal{I}_{W_{s_{0}}^{\mathrm{ani}}}=4\left(2 b_{s}\left(I_{4, s_{0}}-1\right)^{2}+1\right) a_{s} e^{b_{s}\left(I_{4, s_{0}}-1\right)^{2}}\left(\boldsymbol{u} \cdot \boldsymbol{F}_{x} \boldsymbol{s}_{0}\right)^{2}\left(\boldsymbol{V} \cdot \boldsymbol{s}_{0}\right)^{2} \\
&+2 a_{s} e^{b_{f}\left(I_{4, s_{0}}-1\right)^{2}}\left(I_{4, s_{0}}-1\right)(\boldsymbol{u} \cdot \boldsymbol{u})\left(\boldsymbol{V} \cdot \boldsymbol{s}_{0}\right)^{2} \\
& \geq-2 a_{s} e^{b_{s}\left(I_{4, s_{0}}-1\right)^{2}}\left|\left(I_{4, s_{0}}-1\right)\right|(\boldsymbol{u} \cdot \boldsymbol{u})\left(\boldsymbol{V} \cdot \boldsymbol{s}_{0}\right)^{2} .
\end{aligned}
$$

The total contribution $\mathcal{I}_{W}$ when not considering the Macauly brackets is

$$
\begin{aligned}
\mathcal{I}_{W} & =-2 a_{f s} e^{b_{f s} I_{8, f_{0} s_{0}}^{2}} I_{8, f_{0} s_{0}}(\boldsymbol{u} \cdot \boldsymbol{u})\left|\left(\boldsymbol{V} \cdot \boldsymbol{f}_{0}\right)\left(\boldsymbol{V} \cdot \boldsymbol{s}_{0}\right)\right| . \\
& -2 a_{f} e^{b_{s}\left(I_{4, f_{0}}-1\right)^{2}}\left|\left(I_{4, f_{0}}-1\right)\right|(\boldsymbol{u} \cdot \boldsymbol{u})\left(\boldsymbol{V} \cdot \boldsymbol{f}_{0}\right)^{2} \\
& -2 a_{s} e^{b_{s}\left(I_{4, s_{0}}-1\right)^{2}}\left|\left(I_{4, s_{0}}-1\right)\right|(\boldsymbol{u} \cdot \boldsymbol{u})\left(\boldsymbol{V} \cdot \boldsymbol{s}_{0}\right)^{2} .
\end{aligned}
$$

The larger number of terms in (B.21) as opposed to (B.18) leads therefore in general to a model which is more prone for loss of ellipticity, specially when fibres $f_{0}$ and $s_{0}$ are contracted, namely $I_{4, f_{0}}<0$ and $I_{4, s_{0}}<0$.

\section{References}

[1] World Health Organization, Hearts: Technical Package for Cardiovascular Disease Management in Primary Health Care, World Health Organization, 2016.

[2] E. Wilkins, L. Wilson, K. Wickramasinghe, P. Bhatnagar, J. Leal, R. Luengo-Fernandez, R. Burns, M. Rayner, N. Townsend, European Cardiovascular Disease Statistics, European Heart Network, 2017.

[3] D. Mozaffarian, E.J. Benjamin, A.S. Go, D.K. Arnett, M.J. Blaha, M. Cushman, S.R. Das, S. de Ferranti, J.P. Després, H.J. Fullerton, et al., Heart disease and stroke statistics, Circulation 133 (4) (2016) e38-e360.

[4] G.J. Tortora, B.H. Derrickson, Principles of Anatomy and Physiology, John Wiley \& Sons, 2008.

[5] E. Hsu, A. Muzikant, S. Matulevicius, R. Penland, C. Henriquez, Magnetic resonance myocardial fiber-orientation mapping with direct histological correlation, Am. J. Physiol.-Heart Circulatory Physiol. 274 (5) (1998) H1627-H1634.

[6] A. Nagler, C. Bertoglio, M. Gee, W. Wall, Personalization of cardiac fiber orientations from image data using the unscented kalman filter, in: International Conference on Functional Imaging and Modeling of the Heart, Springer, 2013, pp. 132-140.

[7] S. Rossi, T. Lassila, R. Ruiz-Baier, A. Sequeira, A. Quarteroni, Thermodynamically consistent orthotropic activation model capturing ventricular systolic wall thickening in cardiac electromechanics, Eur. J. Mech.-A/Solids 48 (2014) 129-142.

[8] H. Lombaert, J.M. Peyrat, P. Croisille, S. Rapacchi, L. Fanton, F. Cheriet, P. Clarysse, I. Magnin, H. Delingette, N. Ayache, Human atlas of the cardiac fiber architecture: study on a healthy population, IEEE Trans. Med. Imag. 31 (7) (2012) 1436-1447.

[9] S. Göktepe, E. Kuhl, Electromechanics of the heart: a unified approach to the strongly coupled excitation-contraction problem, Comput. Mech. 45 (2-3) (2010) 227-243.

[10] J. Wong, E. Kuhl, Generating fibre orientation maps in human heart models using Poisson interpolation, Comput. Methods Biomech. Biomed. Eng. 17 (11) (2014) 1217-1226.

[11] J. Vossoughi, R.N. Vaishnav, D.J. Patel, Compressibility of the myocardial tissue, Adv. Bioeng. (1980) 45-48.

[12] F. Yin, C. Chan, R.M. Judd, Compressibility of perfused passive myocardium, Amer. J. Physiol.-Heart Circulatory Physiol. 271 (5) (1996) H1864-H1870.

[13] T.A. Quinn, P. Kohl, Combining wet and dry research: experience with model development for cardiac mechano-electric structure-function studies, Cardiovascular Res. 97 (4) (2013) 601-611.

[14] O. Gültekin, G. Sommer, G.A. Holzapfel, An orthotropic viscoelastic model for the passive myocardium: continuum basis and numerical treatment, Comput. Methods Biomech. Biomed. Eng. 19 (15) (2016) 1647-1664.

[15] H. Demiray, On the constitutive equations of biological materials, J. Appl. Mech. 42 (1) (1975) 242-243.

[16] J. Humphrey, R. Strumpf, F. Yin, Determination of a constitutive relation for passive myocardium: I. A new functional form, J. Biomech. Eng. 112 (3) (1990) 333-339.

[17] B. Smaill, P. Hunter, Structure and function of the diastolic heart: material properties of passive myocardium, in: Theory of Heart, Springer, 1991, pp. 1-29.

[18] Y.C. Fung, Biomechanics: Mechanical Properties of Living Tissues, Springer Science \& Business Media, 2013.

[19] J.M. Guccione, A.D. McCulloch, Finite element modeling of ventricular mechanics, in: Theory of Heart, Springer, 1991 , pp. $121-144$. 
[20] K.D. Costa, J.W. Holmes, A.D. McCulloch, Modelling cardiac mechanical properties in three dimensions, Phil. Trans. R. Soc. A 359 (1783) (2001) 1233-1250.

[21] G.A. Holzapfel, R.W. Ogden, Constitutive modelling of passive myocardium: a structurally based framework for material characterization, Phil. Trans. R. Soc. A 367 (1902) (2009) 3445-3475.

[22] J.E. Marsden, T.J.R. Hughes, Mathematical foundations of elasticity, 1994.

[23] J.M. Ball, Convexity conditions and existence theorems in nonlinear elasticity, Arch. Ration. Mech. Anal. 63 (4) (1976) $337-403$.

[24] J.M. Ball, Energy-minimising configurations in nonlinear elasticity, Arch. Ration. Mech. Anal. 63 (4) (1976) $337-403$.

[25] J.M. Ball, Geometry, Mechanics and Dynamics, Springer, 2002, pp. 3-59.

[26] J. Schröder, P. Neff, Invariant formulation of hyperelastic transverse isotropy based on polyconvex free energy functions, Int. J. Solids Struct. 40 (2003) 401-445.

[27] J. Schröder, P. Neff, D. Balzani, A variational approach for materially stable anisotropic hyperelasticity, Int. J. Solids Struct. 42 (2005) 4352-4371.

[28] R. Ortigosa, A.J. Gil, J. Bonet, C. Hesch, A computational framework for polyconvex large strain elasticity for geometrically exact beam theory, Comput. Mech. 57 (2) (2016).

[29] J. Bonet, A.J. Gil, C.H. Lee, M. Aguirre, R. Ortigosa, A first order hyperbolic framework for large strain computational solid dynamics - Part I: Total Lagrangian isothermal elasticity, Comput. Methods Appl. Mech. Engrg. 283 (2015) 689-732.

[30] J. Bonet, A.J. Gil, R. Ortigosa, A computational framework for polyconvex large strain elasticity, Comput. Methods Appl. Mech. Engrg. 283 (2015) 1061-1094.

[31] J. Bonet, A.J. Gil, R. Ortigosa, On a tensor cross product based formulation of large strain solid mechanics, Int. J. Solids Struct. 84 (2016) 49-63.

[32] A.L. Hodgkin, A.F. Huxley, Propagation of electrical signals along giant nerve fibres, Proc. R. Soc. B (1952) 177-183.

[33] N.A. Trayanova, J.J. Rice, CaRdiac electromechanical models: from cell to organ, Front. Physiol. 2 (2011).

[34] L. Tung, A Bidomain Model for Describing Ischemic Myocardial DC Potentials (Ph.D. thesis), Massachusetts Institute of Technology, 1978.

[35] B.J. Roth, J.P. Wikswo, A bidomain model for the extracellular potential and magnetic field of cardiac tissue, IEEE Trans. Biomed. Eng. (4) (1986) 467-469.

[36] R.M. Bordas, K. Gillow, D. Gavaghan, B. Rodríguez, D. Kay, A bidomain model of the ventricular specialized conduction system of the heart, SIAM J. Appl. Math. 72 (5) (2012) 1618-1643.

[37] L.J. Leon, B.M. Horáček, Computer model of excitation and recovery in the anisotropic myocardium: I. rectangular and cubic arrays of excitable elements, J. Electrocardiol. 24 (1) (1991) 1-15.

[38] S. Göktepe, S. Acharya, J. Wong, E. Kuhl, Computational modeling of passive myocardium, Int. J. Numer. Methods Biomed. Eng. 27 (1) (2011) 1-12.

[39] B. Baillargeon, N. Rebelo, D.D. Fox, R.L. Taylor, E. Kuhl, The living heart project: a robust and integrative simulator for human heart function, Eur. J. Mech.-A/Solids 48 (2014) 38-47.

[40] M. Potse, B. Dubé, J. Richer, A. Vinet, R.M. Gulrajani, A comparison of monodomain and bidomain reaction-diffusion models for action potential propagation in the human heart, IEEE Trans. Biomed. Eng. 53 (12) (2006) 2425-2435.

[41] R. FitzHugh, Mathematical models of threshold phenomena in the nerve membrane, Bull. Math. Biophys. 17 (4) (1955) $257-278$.

[42] F. Fenton, A. Karma, Vortex dynamics in three-dimensional continuous myocardium with fiber rotation: filament instability and fibrillation, Chaos 8 (1) (1998) 20-47.

[43] R.R. Aliev, A.V. Panfilov, A simple two-variable model of cardiac excitation, Chaos Solitons Fractals 7 (3) (1996) $293-301$.

[44] C.H. Luo, Y. Rudy, A dynamic model of the cardiac ventricular action potential. ii. afterdepolarizations, triggered activity, and potentiation., Circulation Res. 74 (6) (1994) 1097-1113.

[45] V. Iyer, R. Mazhari, R.L. Winslow, A computational model of the human left-ventricular epicardial myocyte, Biophys. J. 87 (3) (2004) 1507-1525.

[46] L. Priebe, D.J. Beuckelmann, Simulation study of cellular electric properties in heart failure, Circulation Res. 82 (11) (1998) $1206-1223$.

[47] K. ten Tusscher, D. Noble, P. Noble, A. Panfilov, A model for human ventricular tissue, Amer. J. Physiol.-Heart Circulatory Physiol. 286 (4) (2004) H1573-H1589.

[48] K.H. ten Tusscher, A.V. Panfilov, Alternans and spiral breakup in a human ventricular tissue model, Amer. J. Physiol.-Heart Circulatory Physiol. 291 (3) (2006) H1088-H1100.

[49] A. Bueno-Orovio, E.M. Cherry, F.H. Fenton, Minimal model for human ventricular action potentials in tissue, J. Theoret. Biol. 253 (3) (2008) $544-560$.

[50] L.A. Taber, R. Perucchio, Modeling heart development, J. Elasticity 61 (1) (2000) 165-198.

[51] M.P. Nash, A.V. Panfilov, Electromechanical model of excitable tissue to study reentrant cardiac arrhythmias, Prog. Biophys. Mol. Biol. 85 (2) (2004) 501-522.

[52] J. Wong, S. Göktepe, E. Kuhl, Computational modeling of chemo-electro-mechanical coupling: A novel implicit monolithic finite element approach, Int. J. Numer. Methods Biomed. Eng. 29 (10) (2013) 1104-1133.

[53] J. Wong, S. Göktepe, E. Kuhl, Computational modeling of electrochemical coupling: a novel finite element approach towards ionic models for cardiac electrophysiology, Comput. Methods Appl. Mech. Eng. 200 (45) (2011) 3139-3158.

[54] M. Aguirre, A.J. Gil, J. Bonet, A.A. Carreño, A vertex centred finite volume Jameson-Schmidt-Turkel (JST) algorithm for a mixed conservation formulation in solid dynamics, J. Comput. Phys. 259 (2014) 672-699.

[55] G. Scovazzi, B. Carnes, X. Zeng, A simple, stable and accurate tetrahedral finite element for transient, nearly incompressible, linear and nonlinear elasticity: A dynamic variational multiscale approach, Internat. J. Numer. Methods Engrg. (2015).

[56] M. Cervera, M. Chiumenti, R. Codina, Mixed stabilized finite element methods in nonlinear solid mechanics: Part I: Formulation, Comput. Methods Appl. Mech. Engrg. 199 (2010) 2559-2570. 
[57] T. Elguedj, Y. Bazilevs, V. Calo, T. Hughes, B-bar and F-bar projection methods for nearly incompressible linear and non-linear elasticity and plasticity using higher-order NURBS elements, Comput. Methods Appl. Mech. Engrg. 197 (2008) 2732-2762.

[58] A.J. Gil, R. Ortigosa, A new framework for large strain electromechanics based on convex multi-variable strain energies: variational formulation and material characterisation, Comput. Methods Appl. Mech. Engrg. 302 (2016) 293-328.

[59] R. Ortigosa, A.J. Gil, A new framework for large strain electromechanics based on convex multi-variable strain energies: Conservation laws, hyperbolicity and extension to electro-magneto-mechanics, Comput. Methods Appl. Mech. Engrg. 309 (2016) $202-242$.

[60] R. Ortigosa, A.J. Gil, A new framework for large strain electromechanics based on convex multi-variable strain energies: Finite element discretisation and computational implementation, Comput. Methods Appl. Mech. Engrg. 302 (2016) 329-360.

[61] R. de Boer, Vektor- und Tensorrechnung für Ingenieure, Springer-Verlag, 1982.

[62] S. Rossi, R. Ruiz-Baier, L.F. Pavarino, A. Quarteroni, Orthotropic active strain models for the numerical simulation of cardiac biomechanics, Int. J. Numer. Methods Biomed. Eng. 28 (6-7) (2012) 761-788.

[63] M.P. Nash, P.J. Hunter, Computational mechanics of the heart, J. Elasticity Phys. Sci. Solids 61 (1-3) (2000) $113-141$.

[64] P. Flory, Thermodynamic relations for high elastic materials., Trans. Faraday Soc. 57 (1961) 829-838.

[65] C. Sansour, On the physical assumptions underlying the volumetric-isochoric split and the case of anisotropy, Eur. J. Mech.-A/Solids 27 (1) (2008) 28-39.

[66] D. Nolan, A. Gower, M. Destrade, R. Ogden, J. McGarry, A robust anisotropic hyperelastic formulation for the modelling of soft tissue, J. Mech. Behav. Biomed. Mater. 39 (2014) 48-60.

[67] A.N. Annaidh, M. Destrade, M. Gilchrist, J. Murphy, Deficiencies in numerical models of anisotropic nonlinearly elastic materials, Biomech. Model. Mechanobiol. 12 (4) (2013) 781-791.

[68] J.M. Ball, F. Murat, $\mathrm{W}^{1, p}$-quasiconvexity and variational problems for multiple integrals, J. Funct. Anal. 58 (3) (1984) $225-253$.

[69] G.A. Holzapfel, R.W. Ogden, On the tension-compression switch in soft fibrous solids, Eur. J. Mech.-A/Solids 49 (2015) 561-569.

[70] K. Li, R.W. Ogden, G.A. Holzapfel, Computational method for excluding fibers under compression in modeling soft fibrous solids, Eur. J. Mech.-A/Solids 57 (2016) 178-193.

[71] A. Kamgoué, J. Ohayon, Y. Usson, L. Riou, P. Tracqui, Quantification of cardiomyocyte contraction based on image correlation analysis, Cytometry Part A 75 (4) (2009) 298-308.

[72] J. Strobeck, E. Sonnenblick, Myocardial contractile properties and ventricular performance, Heart Cardiovascular Syst. (1986) 31-49.

[73] A.J. Gil, C.H. Lee, J. Bonet, M. Aguirre, A stabilised Petrov-Galerkin formulation for linear tetrahedral elements in compressible, nearly incompressible and truly incompressible fast dynamics, Comput. Methods Appl. Mech. Engrg. 276 (2014) 659-690.

[74] A.J. Gil, C.H. Lee, J. Bonet, R. Ortigosa, A first order hyperbolic framework for large strain computational solid dynamics - part II: Total lagrangian compressible, nearly incompressible and truly incompressible elasticity, Comput. Methods Appl. Mech. Engrg. 300 (2016) 146-181.

[75] M. Crouzeix, P.A. Raviart, Conforming and nonconforming finite element methods for solving the stationary Stokes equations i, Rev. Française Autom. Inf. Rech. Opér. Math. 7 (R3) (1973) 33-75.

[76] F. Bertrand, M. Gadbois, P. Tanguy, Tetrahedral elements for fluid flow, Internat. J. Numer. Methods Engrg. 33 (6) (1992) $1251-1267$.

[77] R. Ortigosa, A.J. Gil, C.H. Lee, A computational framework for large strain nearly and truly incompressible electromechanics based on convex multi-variable strain energies, Comput. Methods Appl. Mech. Engrg. 310 (2016) 297-334.

[78] S. Land, V. Gurev, S. Arens, C.M. Augustin, L. Baron, R. Blake, C. Bradley, S. Castro, A. Crozier, M. Favino, et al., Verification of cardiac mechanics software: Benchmark problems and solutions for testing active and passive material behaviour, in: Proc. R. Soc. A, 471, (2184) The Royal Society, 2015, p. 20150641.

[79] J.M. Guccione, A.D. McCulloch, L. Waldman, et al., Passive material properties of intact ventricular myocardium determined from a cylindrical model, J. Biomech. Eng. 113 (1) (1991) 42-55.

[80] C.J. Chuong, Y.C. Fung, Residual stress in arteries, in: Front. Biomech., Springer, 1986, pp. 117-129. 